\title{
Gravitational radiation from inspiralling compact objects: Spin-spin effects completed at the next-to-leading post-Newtonian order
}

\author{
Gihyuk Cho®, ${ }^{1, *}$ Brian Pardo $\odot^{2, \dagger}$ and Rafael A. Porto $\oplus^{1, *}$ \\ ${ }^{1}$ Deutsches Elektronen-Synchrotron DESY, Notkestrasse 85, \\ 22607 Hamburg, Germany \\ ${ }^{2}$ Pittsburgh Particle Physics, Astrophysics, and Cosmology Center, \\ Department of Physics and Astronomy, University of Pittsburgh, \\ Pittsburgh, Pennsylvania 15260, USA
}

(Received 28 March 2021; accepted 23 April 2021; published 14 July 2021)

\begin{abstract}
Using the gravitational potential and source multipole moments bilinear in the spins, first computed to next-to-leading order (NLO) in the post-Newtonian expansion within the effective field theory (EFT) framework, we complete here the derivation of the dynamical invariants and flux-balance equations, including energy and angular momentum. We use these results to calculate spin-spin effects in the orbital frequency and accumulated phase to NLO for circular orbits. We also derive the linear momentum and center-of-mass fluxes and associated kick velocity, to the highest relevant post-Newtonian order. We explicitly demonstrate the equivalence between the quadratic-in-spin source multipoles obtained using the EFT formalism and those rederived later with more traditional tools, leading to perfect agreement for spin-spin radiative observables to NLO among both approaches.
\end{abstract}

DOI: 10.1103/PhysRevD.104.024037

\section{INTRODUCTION}

Binary systems composed of spinning compact objects are the primary source of gravitational waves (GWs) that are currently being detected by the LIGO/VIRGO collaboration [1,2], and will remain as key laboratories to explore gravity and the physics of black holes and neutron stars [3-5] (and perhaps even more exotic possibilities [6-12]) with future GW observatories such as LISA [13] and the Einstein Telescope [14]. Yet, both the detection and accurate interpretation of the signal are dependent on precise theoretical predictions for the twobody problem in general relativity. This is notoriously important when spin effects are manifest, e.g., [15], in particular due to the expectation that binary black holes may be rapidly rotating, e.g., [16]. While numerical simulations are required for the late stages of the dynamics, e.g., [17], the post-Newtonian (PN) expansion has provided the groundwork to tackle the weak-field/small-velocity inspiral regime [18-21]. So far, PN studies have been carried out notably in the conservative sector, both for nonspinning [22-41] and spinning bodies [42-55],

\footnotetext{
*gihyuk.cho@desy.de

bap100@pitt.edu

†rafael.porto@desy.de
}

using various tools [18-21]. In particular, the effective field theory (EFT) approach introduced in [56], and later extended in [57] to incorporate rotational degrees of freedom, has been instrumental to reach the present state of the art. ${ }^{1}$ However, in the radiation sector, while the source multipoles needed to obtain the GW fluxes in an adiabatic expansion have been computed in some cases to fourth order in the PN expansion for nonspinning bodies $[89,90]$, the spin-dependent counterparts are known to next-to-next-to-leading order $\left(\mathrm{N}^{2} \mathrm{LO}\right)$ at linear order in spin [91-93], and only to NLO for bilinear in spins contributions [93-95]. ${ }^{2}$ This begs for more accurate computations of radiative observables in the case of spinning bodies.

\footnotetext{
${ }^{1}$ The use of ideas from particle physics has also been reinvigorated lately due the repurposing of novel techniques from the theory of scattering amplitudes, e.g., [58,59]. In parallel with an extension of the EFT approach to the post-Minkowskian regime [60-62], these novel approaches have extended the knowledge of the binary dynamics in the conservative sector, both for nonspinning [63-73] and spinning objects [74-83]. Radiation effects in the post-Minkowskian expansion have also been studied in, e.g., [84-88].

${ }^{2}$ The associated radiation-reaction spin-orbit and spin-spin effects in the dynamics have been computed at leading order in $[96,97]$.
} 
All the necessary quadratic-in-spin source multipoles including finite-size effects were first obtained some time ago in $[93,94]$, together with the spin-spin gravitational potentials $[43,45,46]$, using the EFT formalism developed in $[57,98]$. However, the derivation of the associated GW phase evolution was not carried out until recently. In [99], using the results obtained in [49,93] at linear order in the spin, the gravitational observables including the accumulated phase were computed to NLO and shown to agree with the values first derived in [42,91] with more traditional tools. With the aim to move forward in precision towards higher PN orders, the purpose of this paper is to use the results in $[43,45,46,49,93,94]$ to complete the derivation of the spin-spin contributions to the dynamical invariants and flux-balance laws to NLO in generic configurations, including energy and angular momentum; and use the results to derive spin-spin effects in the orbital frequency and accumulated phase to 3PN for (quasi)circular orbits. For completeness, we also compute the linear momentum and center-of-mass (CM) radiated fluxes to the highest possible PN order using our source multipoles, corresponding to leading spin-spin and NLO linear-in-spin corrections, with which we obtain the associated kick velocity. We find agreement with a previous derivation of the radiated momentum in [100] at linear order in the spin; however, we disagree when it comes to spin-spin contributions, even for the simplest case of binary black holes. We trace the difference to missing terms from finite-size effects. On the other hand, we readily demonstrate the equivalence between the source multipole moments computed in $[93,94]$ within the EFT framework and those rederived later in [95] using the approach in [42,91], yielding complete agreement for the linear and bilinear in spin radiative observables at NLO order among both formalisms. Our results here can be used to improve current waveform models for spinning bodies, notably for ellipticlike orbits [101-103]. The derivation of the $\mathrm{N}^{2} \mathrm{LO}$ phase evolution is underway.

This paper is organized as follows. In Sec. II we briefly overview the steps to compute the necessary ingredients to obtain the GW phase evolution in the EFT framework. In Sec. III we start in the conservative sector and use the gravitational potential in $[43,45,46]$ to compute the conserved energy and angular momentum, as well as the position of the CM, at quadratic order in the spins and to NLO in the PN expansion. In Sec. IV we move to the radiation sector and compute the associated flux-balance equations using the source multipole moments bilinear in spin obtained in $[93,94]$. We also calculate the radiated fluxes of linear momentum and position of the $\mathrm{CM}$, and derive the kick velocity for (quasi)circular orbits. In Sec. V we use the results from previous sections to compute the bilinear-in-spin accumulated phase to 3PN order. We conclude in Sec. VI with a few remarks on future work. We include several appendixes with the coefficients of the lengthy expressions quoted in the main text. For the reader's convenience we add an ancillary file with the main results of the paper [104].

\section{EFT SETUP}

Both the gravitational conservative dynamics and radiative multipole moments of spinning binary systems can be obtained using the EFT formalism introduced in $[43,45,46,49,56,57,93,94,98,105]$. We provide here a brief overview of the EFT framework and encourage the reader to consult the more comprehensive reviews in $[20,21,106]$ for further details.

\section{A. Point-particle theory}

The main actor in the EFT approach is the point-particle action,

$$
S_{\mathrm{pp}}=\sum_{A=\{1,2\}} \int \mathcal{R}_{A} d \sigma_{A},
$$

with $\sigma_{A}$ an affine parameter, which for the case of spinning bodies is written in terms of a Routhian,

$$
\begin{aligned}
\mathcal{R}_{A}= & -\sum_{A}\left[m_{A} \sqrt{v_{A}^{2}}+\frac{1}{2} \omega_{\mu a b} S_{A}^{a b} v_{A}^{\mu}\right. \\
& +\frac{1}{2 m_{A}} R_{\nu c a b} S_{A}^{a b} v_{A}^{\nu} S_{A}^{c d} v_{A d} \\
& \left.-\frac{C_{E S^{2}}^{(A)}}{2 m_{A}} \frac{E_{a b}}{\sqrt{v_{A}^{2}}} S_{A}^{a c} S_{A c}{ }^{b}+\cdots\right],
\end{aligned}
$$

displayed here to quadratic order in the spins. The velocity is defined as $v^{\mu} \equiv \frac{d x^{\mu}}{d \sigma}$, and $\omega_{\mu}^{a b}$ are the Ricci rotation coefficients. The spin tensor, $S^{\mu \nu}$, has been projected onto a locally flat frame, described by a tetrad field, $e_{a}^{\mu}$, such that $S^{a b} \equiv e_{\mu}^{a} e_{\nu}^{b} S^{\mu \nu}$. There are also two curvature-dependent terms. The first ensures the preservation of the covariant spin supplementary condition (SSC), to the order we work here,

$$
S_{A}^{a b} v_{A b}=S_{A}^{a b} e_{b}^{\mu} v_{A \mu}=0+\mathcal{O}\left(S^{3}\right)
$$

The other one, depending on the electric component of the Weyl tensor, $E_{\mu \nu}$, encapsulates the spin-induced quadrupole moment of a rotating object. The $C_{E S^{2}}^{(A)}$ parameters are socalled Wilson coefficients, which carry information about the short-distance physics of the compact body, e.g., $C_{E S^{2}}=1$ for Kerr black holes $[45,46]$. The ellipsis contains an additional hierarchy of higher curvature corrections that include other finite-size multipolar corrections, beyond the quadrupole, as well as extra pieces required to enforce the SSC.

The equations of motion (EOM) for each particle can then be obtained via 


$$
\frac{\delta S_{\mathrm{pp}}}{\delta x_{A}^{\mu}}, \quad \frac{d S_{A}^{a b}}{d \sigma_{A}}=\left\{S_{A}^{a b}, \mathcal{R}_{A}\right\}
$$

with the spin algebra

$$
\left\{S_{A}^{a b}, S_{A}^{c d}\right\}=\eta^{a c} S_{A}^{b d}+\eta^{b d} S_{A}^{a c}-\eta^{a d} S_{A}^{b c}-\eta^{b c} S_{A}^{a d} .
$$

After the dynamical equations are obtained, the SSC in (2) may be enforced, enabling us to rewrite the EOM in vectorial form. To the order we work in this paper, we have $(B \neq A)[45,46,49]$

$$
\begin{aligned}
S_{A}^{i 0}= & \left(\boldsymbol{v}_{A} \times \boldsymbol{S}_{A}\right)^{i}+\frac{2 G m_{B}}{r}\left(\left(\boldsymbol{v}_{A}-\boldsymbol{v}_{B}\right) \times \boldsymbol{S}_{A}\right)^{i} \\
& +\frac{G}{r^{3}}\left(\boldsymbol{S}_{B}^{i} \boldsymbol{r} \cdot \boldsymbol{S}_{A}-\boldsymbol{r}^{i} \boldsymbol{S}_{A} \cdot \boldsymbol{S}_{B}\right)+\cdots,
\end{aligned}
$$

where $\boldsymbol{r} \equiv \boldsymbol{x}_{A}-\boldsymbol{x}_{B}$, and the spin three-vector is defined as

$$
S_{A}^{i} \equiv \frac{1}{2} \epsilon^{i j k} S_{A}^{j k}
$$

\section{B. Long-distance worldline action}

For the two-body problem in the inspiral regime the gravitational field is expanded around flat space in the weak-field approximation,

$$
g_{\mu \nu}=\eta_{\mu \nu}+h_{\mu \nu}
$$

where $h_{\mu \nu}$ is further split into potential, $H_{\mu \nu}$, and radiation, $\bar{h}_{\mu \nu}$, modes scaling as $\left(k^{0}, \boldsymbol{k}\right)_{\mathrm{pot}} \sim(v / r, 1 / r)$ and $\left(k^{0}, \boldsymbol{k}\right)_{\mathrm{rad}} \sim$ $(v / r, v / r)$, respectively [56]. The long-distance effective theory is obtained by "integrating out" the (off-shell) potential modes in a (classical) saddle-point approximation, and matching into a "long-distance" worldline theory, describing now the entire binary and depending only on the (on-shell) radiation field. The effective action takes the generic form $[98,105]^{3}$

$$
\begin{aligned}
S_{\mathrm{rad}}= & \int d \sigma \sqrt{\left(\eta_{\mu \nu}+\bar{h}_{\mu \nu}\right) V^{\mu} V^{\nu}}\left[-P^{\mu}(\sigma) V^{\nu}(\sigma)\left(1+\boldsymbol{X}^{i} \nabla_{i}\right) \bar{h}_{\mu \nu}(\sigma, \boldsymbol{X}(\sigma))-\frac{1}{2} \bar{\omega}_{\mu}^{\alpha \beta}(\sigma, \boldsymbol{X}) J_{\alpha \beta}(\sigma) V^{\mu}(\sigma)\right. \\
& \left.+\sum_{\ell=2}\left(\frac{1}{\ell !} I_{\mathrm{STF}}^{L}(\sigma) \nabla_{L-2} \bar{E}_{i_{\ell-1} i_{\ell}}(\sigma, \boldsymbol{X}(\sigma))-\frac{2 \ell}{(2 \ell+1) !} J_{\mathrm{STF}}^{L}(\sigma) \nabla_{L-2} \bar{B}_{i_{\ell-1} i_{\ell}}(\sigma, \boldsymbol{X}(\sigma))\right)\right],
\end{aligned}
$$

around the $\mathrm{CM}, \boldsymbol{X}(\sigma)$, of the binary, with $\sigma$ an affine parameter. Throughout this paper we use the notation $L \equiv i_{1} \cdots i_{L}$. All the barred quantities are evaluated on $\bar{h}_{\mu \nu}$. The first terms represent the total four momentum, $P^{\mu}$, and angular momentum, $J^{\alpha \beta}(t)$, of the binary, with $V^{\mu}$ its $\mathrm{CM}$ velocity. The higher-order multipole moments have electric-type, $I^{L}(t)$, and magnetic-type, $J^{L}(t)$, parity and couple to derivatives of the electric and magnetic components of the Weyl tensor, respectively. We have also kept the term proportional to $\boldsymbol{X}^{i}$ at linear order in derivatives, which will be useful later on to compute the position of the $\mathrm{CM}$. For most of the calculations, however, it is sufficient to have the binary at the origin, i.e., $\boldsymbol{X}^{i}=0$.

\section{Gravitational potential}

The gravitational potential (along with the kinetic term) may be obtained by matching the 00 component of the onepoint function. Alternatively, it can be read off from the "vacuum binding energy," where the outgoing radiation

\footnotetext{
${ }^{3}$ Due to the fact that both potential and radiation modes vary on the same timescales, the decoupling of short-distance effects occurs in space but not in time. As a result, the action is endowed with time-dependent "Wilson coefficients." The same type of description can also be used to study absorption effects, e.g., [107-109].
}

field is zero to zero. ${ }^{4}$ Following the latter option, the relevant topologies are shown in Fig. 1 to NLO, where the dashed line represents the potential mode, whose propagator is expanded as $\left(k^{0} \ll|\boldsymbol{k}|\right)$

$$
\frac{i}{\left(k^{0}\right)^{2}-\boldsymbol{k}^{2}}=-\frac{i}{\boldsymbol{k}^{2}}\left(1+\frac{\left(k^{0}\right)^{2}}{\boldsymbol{k}^{2}}+\cdots\right)
$$

and subsequently truncated to the desired PN order. A similar power counting also applies to the nonlinear couplings involving time derivatives of the $H_{\mu \nu}$ field, scaling as $\partial_{0} H_{\mu \nu} \sim(v / r) H_{\mu \nu}$. The Feynman rules for the worldline vertices follow from the action/Routhian in (1), and include mass, spin, as well as finite-size and SSCpreserving contributions, which are likewise also PN expanded in powers of the velocity. Once the potential is known, the EOM follow from (3), which can afterwards be reduced to vectorial form using the SSC in (5). In principle, the gravitational potential also depends on time derivatives of the position and spin variables. As is standard, these are

\footnotetext{
${ }^{4}$ In principle, radiation-reaction effects also contribute to the conservative binding energy through tail effects, e.g., [25,110]. However, to the order we work in this paper, we can safely ignore these types of contributions.
} 


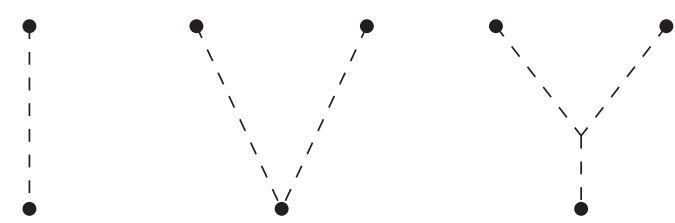

FIG. 1. Topologies needed to match the gravitational potential to NLO (see text).

either integrated by parts or reduced using lowerorder EOM.

The procedure described above was carried out in $[43,45,46,49,57]$ to NLO in the PN expansion and to quadratic order in the spins. In Appendix A we provide expressions for the acceleration and spin EOM in the $\mathrm{CM}$ frame to $3 \mathrm{PN}$ order.

\section{Multipole moments}

It is convenient, in order to compute the multipole moments, to first obtain their generic dependence on moments of the (pseudo-)stress-energy tensor, which includes contributions from potential modes, and subsequently read off the latter via a matching computation with the one-point function (see $[98,105]$ for details on this procedure). The necessary topologies to NLO are shown in Fig. 2 where, once again, the Feynman rules for the vertices follow from (1). In addition to the potential modes, which are integrated out, the wavy line represents the radiation field that propagates to infinity. ${ }^{5}$ Importantly, for the (momentum-conserving) interaction in the nonlinear cubic coupling, between an incoming potential mode with threemomentum $\boldsymbol{q}$ and an outgoing radiation field with threemomentum $\boldsymbol{k}$, in addition to the expansion of potentials in quasi-instantaneous interaction we alluded before in (9) we must also expand the outgoing potential mode as [56]

$$
\frac{i}{(\boldsymbol{k}+\boldsymbol{q})^{2}}=\frac{i}{\boldsymbol{q}^{2}}\left(1-2 \frac{\boldsymbol{q} \cdot \boldsymbol{k}}{\boldsymbol{q}^{2}}+\cdots\right)
$$

At the level of the action, the above is related to a Taylor expansion in (spatial) derivatives of the radiation field around the CM of the binary (placed at the origin) $[56,98]$

$$
\bar{h}_{\mu \nu}(t, \boldsymbol{x})=\bar{h}_{\mu \nu}(t, \mathbf{0})+\partial_{i} \bar{h}_{\mu \nu}(t, \mathbf{0}) \boldsymbol{x}^{i}+\cdots
$$

The procedure described here was carried out in $[93,94]$ to NLO in the PN expansion, and to linear and bilinear order in the spins. Together with the gravitational potential, the multipole moments are the last ingredient to compute all

\footnotetext{
${ }^{5}$ Notice that the leading contribution is simply from the mass coupling to the radiation field in (1). This means that the NLO correction entails only "tree-level" potential exchanges, unlike the derivation of the gravitational potential in Fig. 1, which requires "one-loop" diagrams.
}

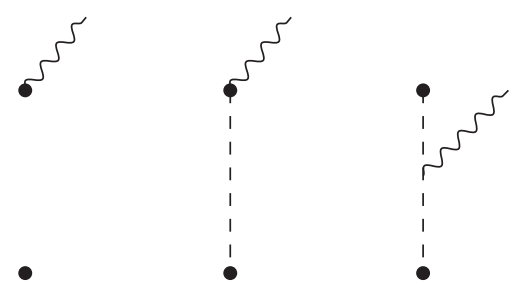

FIG. 2. Topologies needed to match the one-point function to NLO (see text).

the GW fluxes. For completeness, we summarize in Appendix A the resulting multipoles in the CM frame.

\section{ADIABATIC INVARIANTS}

Using the results for the gravitational potential it is straightforward to compute the conserved quantities of the system, which do not evolve in time when radiation fluxes are turned off. These are the objects, such as the binding energy and total angular momentum, that will be part of the balance equations in an adiabatic expansion, valid during the inspiral regime. It is somewhat convenient to express the values for these quantities in the CM frame. This is achieved by first computing the binary's CM, the same way we extract the multipoles, and then solving for the coordinates using the condition $\boldsymbol{X}^{i}=0$. Using the notation $\boldsymbol{r} \equiv \boldsymbol{x}_{1}-\boldsymbol{x}_{2}$ and $\boldsymbol{v} \equiv \dot{\boldsymbol{r}}$, we have solutions that can be PN expanded in the form

$$
\begin{aligned}
& \mathbf{x}_{1}^{i}=\frac{m_{2}}{m} \boldsymbol{r}^{i}+\delta \boldsymbol{r}_{1 \mathrm{PN}}^{i}+\delta \boldsymbol{r}_{\mathrm{LO}-\mathrm{SO}}^{i}+\delta \boldsymbol{r}_{\mathrm{LO}-\mathrm{SO}}^{i}+\cdots, \\
& \boldsymbol{v}_{1}^{i}=\frac{m_{2}}{m} \boldsymbol{v}^{i}+\delta \dot{\boldsymbol{r}}_{1 \mathrm{PN}}^{i}+\delta \dot{r}_{\mathrm{LO}-\mathrm{SO}}^{i}+\delta \dot{\boldsymbol{r}}_{\mathrm{LO}-\mathrm{SO}}^{i}+\cdots,
\end{aligned}
$$

with $m \equiv m_{1}+m_{2}$, and similarly for the companion. To the order we work in this paper we find the known values (e.g., $[42,111])$,

$$
\begin{gathered}
\delta \boldsymbol{r}_{1 \mathrm{PN}}^{i}=\frac{\nu \delta}{2} \boldsymbol{r}^{i}\left(\boldsymbol{v}^{2}-\frac{G m}{r}\right), \\
\delta \boldsymbol{r}_{\mathrm{LO}-\mathrm{SO}}^{i}=\frac{\nu}{m}(\boldsymbol{v} \times \boldsymbol{\Sigma})^{i}, \\
\delta \boldsymbol{r}_{\text {LO-SO }}^{i}=0,
\end{gathered}
$$

with $\nu \equiv \frac{m_{1} m_{2}}{m^{2}}, \delta \equiv \frac{m_{1}-m_{2}}{m}$, and

$$
\boldsymbol{\Sigma} \equiv m\left(\frac{\boldsymbol{S}_{2}}{m_{2}}-\frac{\boldsymbol{S}_{1}}{m_{1}}\right)
$$

while for the velocities we have

$$
\delta v_{1 \mathrm{PN}}^{i}=\frac{\delta \nu}{2} \boldsymbol{v}^{i}\left(\boldsymbol{v}^{2}-\frac{G m}{r}\right)-\frac{G m \delta \nu}{2 r} \dot{r} n^{i}
$$




$$
\delta v_{\mathrm{LO}-\mathrm{SO}}^{i}=-\frac{G \nu}{r^{2}}(\boldsymbol{n} \times \boldsymbol{\Sigma})^{i},
$$

with $\boldsymbol{n} \equiv \boldsymbol{r} / r$. The latter expressions follow by taking time derivatives of the position and using lower-order EOM.

For the remainder of the paper we will often quote the final results in terms of (13d) and the total spin

$$
S \equiv S_{1}+S_{2}
$$

For completeness, we also provide expressions for the 3PN correction at quadratic order in the spins in Sec. III C, which will be useful to express conserved quantities in the CM frame at higher orders. The NLO spin-orbit correction was computed in [99].

\section{A. Binding energy}

From the gravitational potential we can derive the binding energy following the standard Euler-Lagrange procedure, yielding

$$
\begin{aligned}
E= & E_{\mathrm{N}}+E_{1 \mathrm{PN}}+E_{\mathrm{LO}-\mathrm{SO}}+\left[E_{2 \mathrm{PN}}+E_{\mathrm{LO}-\mathrm{SS}}\right]+E_{\mathrm{NLO}-\mathrm{SO}} \\
& +\left[E_{3 \mathrm{PN}}+E_{\mathrm{NLO}-\mathrm{SS}}\right]+\cdots
\end{aligned}
$$

in a PN expansion. The computation is often lengthy but straightforward. Using the potential in [49], the spin-orbit binding energy in the CM at NLO was computed in [99], confirming the old result in [42]. We compute here, using the results first obtained in $[43,45,46]$, the contribution to the $\mathrm{CM}$ binding energy to $3 \mathrm{PN}$ and quadratic order in the spins. We find

$$
\begin{gathered}
E_{\mathrm{LO}-\mathrm{SS}}=\frac{G \nu}{r^{3}} \frac{1}{4} e_{4}^{0}, \\
E_{\mathrm{NLO}-\mathrm{SS}}=\frac{G \nu}{r^{3}}\left[\frac{1}{8} e_{6}^{0}+\frac{G m}{r} \frac{1}{4} e_{6}^{1}\right],
\end{gathered}
$$

where the $e_{4}^{0}, e_{6}^{0}, e_{6}^{1}$ coefficients are displayed in Appendix B. After rewriting the answer in terms of the conserved-norm spin variable, ${ }^{6}$

$$
\boldsymbol{S}_{c}=\left(1-\frac{\boldsymbol{v}^{2}}{2}\right) \boldsymbol{S}+\frac{1}{2}(\boldsymbol{v} \cdot \boldsymbol{S}) \boldsymbol{v}+\cdots,
$$

the NLO result in (16) is completely equivalent to the derivation in [95]. This is expected, since the results in [95] for the binding energy are obtained after confirming the equivalence with the value of the gravitational potential first computed in $[45,46])$.

\footnotetext{
${ }^{6} \mathrm{We}$ only quote the terms which are needed to match the multipoles. The reader should keep in mind, however, that higherorder corrections are necessary to achieve a precession form for the spin EOM; see, e.g., [45,46,49].
}

\section{Nonprecessing (quasi)circular orbits}

Many of the above expressions drastically simplify for the case of circular orbits, which we expect to provide a large fraction of the relevant sources of GWs once the binary enters the frequency band of present and future ground-based detectors. We proceed using the basis of vectors $\{\boldsymbol{n}, \boldsymbol{\lambda}, \boldsymbol{l}\}$, defined by

$$
\begin{aligned}
\ell & \equiv \frac{r \times v}{|r \times v|}, \\
\lambda & \equiv \ell \times n,
\end{aligned}
$$

with $\boldsymbol{n}$ as the radial unit vector. Following the nonspinning case, we seek (quasi)circular orbits obeying

$$
\begin{gathered}
\frac{d \boldsymbol{n}}{d t}=\omega(t) \lambda, \\
\frac{d \lambda}{d t}=-\omega(t) \boldsymbol{n}, \\
\frac{d \boldsymbol{\ell}}{d t}=0 .
\end{gathered}
$$

The reader will immediately notice that these conditions cannot be fulfilled when spin effects are included, since only the total angular momentum is conserved. ${ }^{7}$ Hence, we must enforce additional constraints in order to find (quasi) circular orbits. We use the "aligned-spin" simplifications

$$
\begin{gathered}
\boldsymbol{n} \cdot \boldsymbol{S}_{A}=0, \\
\lambda \cdot \boldsymbol{S}_{A}=0, \\
\mathbf{S}_{1} \times \mathbf{S}_{2}=0,
\end{gathered}
$$

to ensure the orbit is confined to the plane. In order to guarantee these are valid throughout the entire evolution of the binary, their time derivatives must also be consistent with the EOM to the desired PN order. The above conditions then imply that the spin vectors must be aligned with the orbital angular momentum,

$$
S_{A} \equiv S_{A} \ell
$$

and remain constant in time $\left(\dot{S}_{1,2}=0\right){ }^{8}$ Moreover, because the radius is also constant $(\dot{r}=0)$, there must be a direct

\footnotetext{
${ }^{7}$ When only spin-orbit corrections are included at leading order, orbits with $\dot{r}=0$ are still possible but not restricted to the plane.

${ }^{8}$ Notice that the covariant spin vector varies with time in generic orbits. However, since $\dot{\boldsymbol{v}}^{2}=0$ for (quasi)circular orbits when radiation-damping is omitted, the difference between $S$ and $\boldsymbol{S}_{c}$ in (17) is simply an overall rescaling. Therefore, both vectors remain constant in time when initially aligned with the orbital angular momentum.
} 
relationship between the latter and the orbital frequency, which also obeys $\dot{\omega}=0$ as long as fluxes are ignored. Introducing the PN parameter $x \equiv(G m \omega)^{2 / 3}$, we find

$$
\left(\frac{G m}{r}\right)_{\mathrm{N}+1 \mathrm{PN}+\mathrm{LO}-\mathrm{SO}}=x+x^{2}\left(1-\frac{\nu}{3}\right)+\frac{x^{5 / 2}}{G m^{2}}\left(\frac{5}{3} S+\delta \Sigma\right),
$$

and for the spin-spin contributions we have

$$
\begin{aligned}
\left(\frac{G m}{r}\right)_{\mathrm{SS}}= & \frac{x^{3}}{4 G^{2} m^{4}}\left[\left(-4-2 \kappa_{+}\right) S^{2}+\left(-4 \delta+2 \kappa_{-}-2 \delta \kappa_{+}\right)(S \Sigma)+\left(\delta \kappa_{-}-\kappa_{+}+\left(4+2 \kappa_{+}\right) \nu\right) \boldsymbol{\Sigma}^{2}\right] \\
& +\frac{x^{4}}{36 G^{2} m^{4}}\left[\left(74-42 \delta \kappa_{-}-24 \kappa_{+}+\left(-48-24 \kappa_{+}\right) \nu\right) S^{2}\right. \\
& +\left(78 \delta-18 \kappa_{-}+18 \delta \kappa_{+}+\left(-48 \delta+192 \kappa_{-}-24 \delta \kappa_{+}\right) \nu\right)(S \Sigma) \\
& \left.+\left(36-9 \delta \kappa_{-}+9 \kappa_{+}+\left(-90+54 \delta \kappa_{-}-72 \kappa_{+}\right) \nu+\left(48+24 \kappa_{+}\right) \nu^{2}\right) \boldsymbol{\Sigma}^{2}\right],
\end{aligned}
$$

where we use the notation $(A B) \equiv \boldsymbol{A} \cdot \boldsymbol{B}$ (for $\boldsymbol{A} \neq \boldsymbol{B}$ ) adopted in, e.g., [95]. We also introduced the finite-size parameters $\kappa_{ \pm} \equiv C_{E S^{2}}^{(1)} \pm C_{E S^{2}}^{(2)}$. Replacing the expression (23) into (16), we finally get the binding energy for (quasi)circular orbits as a function of the orbital frequency,

$$
\begin{aligned}
(E)_{\mathrm{SS}}= & \frac{x^{3} \nu}{4 G^{2} m^{3}}\left(\left(4+2 \kappa_{+}\right) S^{2}+\left(4 \delta-2 \kappa_{-}+2 \delta \kappa_{+}\right)(S \Sigma)+\left(-\delta \kappa_{-}+\kappa_{+}+\left(-4-2 \kappa_{+}\right) \nu\right)\right) \Sigma^{2} \\
& +\frac{x^{4} \nu}{72 G^{2} m^{3}}\left(\left(-236+78 \delta \kappa_{-}+132 \kappa_{+}+\left(12+6 \kappa_{+}\right) \nu\right) S^{2}\right. \\
& +\left(-336 \delta-54 \kappa_{-}+54 \delta \kappa_{+}+\left(12 \delta-318 \kappa_{-}+6 \delta \kappa_{+}\right) \nu\right)(S \Sigma) \\
& \left.+\left(-180-27 \delta \kappa_{-}+27 \kappa_{+}+\left(396-81 \delta \kappa_{-}+27 \kappa_{+}\right) \nu+\left(-12-6 \kappa_{+}\right) \nu^{2}\right) \Sigma^{2}\right) .
\end{aligned}
$$

We can also transform the above expression as function of the conserved-norm spin vector. Using (17) we have

$$
\begin{aligned}
(E)_{\mathrm{SS}}= & \frac{x^{3} \nu}{4 G^{2} m^{3}}\left[\left(4+2 \kappa_{+}\right) \boldsymbol{S}_{c}^{2}+\left(4 \delta-2 \kappa_{-}+2 \delta \kappa_{+}\right)\left(S_{c} \Sigma_{c}\right)+\left(-\delta \kappa_{-}+\kappa_{+}+\left(-4-2 \kappa_{+}\right) \nu\right) \boldsymbol{\Sigma}_{c}^{2}\right] \\
& +\frac{x^{4} \nu}{72 G^{2} m^{3}}\left[\left(-200+60 \delta \kappa_{-}+150 \kappa_{+}+\left(-60-30 \kappa_{+}\right) \nu\right) S_{c}^{2}\right. \\
& +\left(-300 \delta-90 \kappa_{-}+90 \delta \kappa_{+}+\left(-60 \delta-210 \kappa_{-}-30 \delta \kappa_{+}\right) \nu\right)\left(S_{c} \Sigma_{c}\right) \\
& \left.+\left(-180-45 \delta \kappa_{-}+45 \kappa_{+}+\left(360-45 \delta \kappa_{-}-45 \kappa_{+}\right) \nu+\left(60+30 \kappa_{+}\right) \nu^{2}\right) \boldsymbol{\Sigma}_{c}^{2}\right]
\end{aligned}
$$

which agrees with the result in [95].

\section{B. Orbital angular momentum}

The orbital angular momentum can be obtained either by matching the one-point function to the long-distance action in (8), or by obtaining the Noether current using the gravitational potential. Following the latter, we arrive at an expression that can likewise be PN expanded as

$$
\begin{aligned}
\boldsymbol{L}^{i}= & \boldsymbol{L}_{\mathrm{N}}^{i}+\boldsymbol{L}_{1 \mathrm{PN}}^{i}+\boldsymbol{L}_{\mathrm{LO}-\mathrm{SO}}^{i}+\left[\boldsymbol{L}_{\mathrm{2PN}}^{i}+\boldsymbol{L}_{\mathrm{LO}-\mathrm{SS}}^{i}\right]+\boldsymbol{L}_{\mathrm{NLO}-\mathrm{SO}}^{i} \\
& +\left[\boldsymbol{L}_{3 \mathrm{PN}}^{i}+\boldsymbol{L}_{\mathrm{NLO}-\mathrm{SS}}^{i}\right]+\cdots
\end{aligned}
$$

where

$$
\begin{gathered}
\boldsymbol{L}_{\mathrm{N}}^{i}=\nu m r(\boldsymbol{n} \times \boldsymbol{v})^{i}, \\
\boldsymbol{L}_{1 \mathrm{PN}}^{i}=\nu m r(\boldsymbol{n} \times \boldsymbol{v})^{i}\left(\frac{G m(3+\nu)}{r}+\frac{(1-3 \nu) \mathbf{v}^{2}}{2}\right),
\end{gathered}
$$

$$
\boldsymbol{L}_{\mathrm{LO}-\mathrm{SO}}^{i}=\frac{G m \nu}{r}\left(\boldsymbol{n}^{i}(3(n S)+\delta(n \Sigma))-3 \boldsymbol{S}^{i}-\delta \boldsymbol{\Sigma}^{i}\right)
$$

$$
\boldsymbol{L}_{\mathrm{LO}-\mathrm{SS}}^{i}=0
$$


At 2.5PN and 3PN order, respectively, we have

$$
\begin{gathered}
\boldsymbol{L}_{\mathrm{NLO}-\mathrm{SO}}^{i}=\frac{G m \nu}{2 r}\left[\ell_{5}^{0}+\frac{G m}{r} \ell_{5}^{1}\right], \\
\boldsymbol{L}_{\mathrm{NLO}-\mathrm{SS}}^{i}=\frac{G m \nu}{2 r^{2}} \ell_{6}^{0} .
\end{gathered}
$$

The lengthy expression for the $\ell_{5}^{0}, \ell_{5}^{1}$, and $\ell_{6}^{0}$ coefficients can be found in Appendix B. Notice that in the orbital angular momentum, there is no spin-spin contribution at 2PN order. The 3PN expression for the spin-spin orbital angular momentum is presented here for the first time. We have explicitly confirmed that the evolution of the total angular momentum obeys

$$
\frac{d}{d t}\left(\boldsymbol{L}^{i}+\boldsymbol{S}^{i}\right)=0
$$

using the EOM deriving from the gravitational potential to $3 \mathrm{PN}$ order $[43,45,46,49,57]$, which provides a nontrivial check of our result.

\section{Center-of-mass position}

Finally, we also compute the correction to the CM position. This is achieved, as we discussed in Sec. III, by matching the one-point function to the effective action in (8) and reading off the position of the $\mathrm{CM}, \boldsymbol{X}^{i}$, by expanding in the soft-radiation mode at linear order $\boldsymbol{k}$. We arrive at

$$
\left(\boldsymbol{X}^{i}\right)_{\mathrm{LO}-\mathrm{SS}}=0
$$

from which we find the correction to (12a) given by

$$
\begin{aligned}
\left(\delta \boldsymbol{r}^{i}\right)_{\mathrm{NLO}-\mathrm{SS}}= & \boldsymbol{S}^{i}\left(12 \kappa_{-}(n S)+\left(12+6 \delta \kappa_{-}-6 \kappa_{+}\right)(n \Sigma)\right)+\boldsymbol{\Sigma}^{i}\left[\left(-4+6 \delta \kappa_{-}-6 \kappa_{+}\right)(n S)+\left(-6 \delta \kappa_{+}+\kappa_{-}(6-12 \nu)\right)(n \Sigma)\right] \\
& +\mathbf{n}^{i}\left[\left(12 \delta+6 \delta \kappa_{+}\right)(n S)^{2}+\left(12 \delta^{2}-6 \delta \kappa_{-}+6 \delta^{2} \kappa_{+}\right)(n S)(n \Sigma)\right. \\
& +\left(-3 \delta^{2} \kappa_{-}+\delta \kappa_{+}(3-6 \nu)-12 \delta \nu\right)(n \Sigma)^{2}+\left(-4 \delta-4 \kappa_{-}-2 \delta \kappa_{+}\right) S^{2} \\
& \left.+\left(-2 \delta \kappa_{-}+4(-3+4 \nu)+\kappa_{+}(2+8 \nu)\right)(S \Sigma)+\left(-\kappa_{-}+4 \delta \nu+\kappa_{+}(\delta+2 \delta \nu)\right) \boldsymbol{\Sigma}^{2}\right] .
\end{aligned}
$$

\section{FLUX-BALANCE LAWS}

In this section we use the source multipole moments from [93] together with the EOM in $[43,45,46,49,57]$ to compute the flux-balance equations for the binding energy, linear momentum, and angular momentum to 3PN order. We do not include the contribution from the (spin-orbit) tail term, which corrects the radiative multipole moments [93,94], whose effect can be found in, e.g., [112]. The detailed expressions for generic orbits are lengthy and relegated to Appendix C. Explicit results are given in the main text for the case of nonprecessing (quasi) circular orbits.

\section{A. Binding energy}

For the computation of the energy flux we use the wellknown formula, e.g., [98]

$$
\begin{aligned}
\frac{d E}{d t}= & -\frac{G}{5}\left(I_{i j}^{(3)} I_{i j}^{(3)}+\frac{16}{9} J_{i j}^{(3)} J_{i j}^{(3)}+\frac{5}{189} I_{i j k}^{(4)} I_{i j k}^{(4)}\right. \\
& \left.+\frac{5}{84} J_{i j k}^{(4)} J_{i j k}^{(4)}+\cdots\right),
\end{aligned}
$$

in terms of the source multipole moments. The explicit expression for the latter are given in Appendix A in the CM frame. The time derivatives are order-reduced using the EOM, also found in Appendix A. The result can be expanded in the PN expansion,

$$
\begin{aligned}
\frac{d E}{d t}= & \left(\frac{d E}{d t}\right)_{\mathrm{N}}+\left(\frac{d E}{d t}\right)_{1 \mathrm{PN}}+\left(\frac{d E}{d t}\right)_{\text {LO-SO }} \\
& +\left[\left(\frac{d E}{d t}\right)_{2 \mathrm{PN}}+\left(\frac{d E}{d t}\right)_{\mathrm{LO}-\mathrm{SS}}\right]+\left(\frac{d E}{d t}\right)_{\mathrm{NLO}-\mathrm{SO}} \\
& +\left[\left(\frac{d E}{d t}\right)_{3 \mathrm{PN}}+\left(\frac{d E}{d t}\right)_{\mathrm{NLO}-\mathrm{SS}}\right]+\cdots,
\end{aligned}
$$


and keeping only spin-spin contributions to the leading order, we find

$$
\begin{aligned}
\left(\frac{d E}{d t}\right)_{\text {LO-SS }}= & \frac{G^{3} m^{2} \nu^{2}}{105 r^{6}}\left[\left(696+348 \kappa_{+}\right)(n S)(n v)(v S)\right. \\
& +\left(348 \delta-174 \kappa_{-}+174 \delta \kappa_{+}\right)(n v)(n \Sigma)(v S)+\left(-144-72 \kappa_{+}\right)(v S)^{2} \\
& +S^{2}\left(\left(312+156 \kappa_{+}\right)(n v)^{2}+\left(-288-144 \kappa_{+}\right) \boldsymbol{v}^{2}\right) \\
& +(n S)^{2}\left(\left(-1632-816 \kappa_{+}\right)(n v)^{2}+\left(1008+504 \kappa_{+}\right) \boldsymbol{v}^{2}\right) \\
& +(S \Sigma)\left(\left(312 \delta-156 \kappa_{-}+156 \delta \kappa_{+}\right)(n v)^{2}+\left(-288 \delta+144 \kappa_{-}-144 \delta \kappa_{+}\right) \boldsymbol{v}^{2}\right) \\
& +(n S)(n \Sigma)\left(\left(-1632 \delta+816 \kappa_{-}-816 \delta \kappa_{+}\right)(n v)^{2}+\left(1008 \delta-504 \kappa_{-}+504 \delta \kappa_{+}\right) \boldsymbol{v}^{2}\right) \\
& +(n \Sigma)^{2}\left[\left(-9+408 \delta \kappa_{-}+1632 \nu+408 \kappa_{+}(-1+2 \nu)\right)(n v)^{2}+\left(-252 \delta \kappa_{-}+\kappa_{+}(252-504 \nu)-1008 \nu\right) \boldsymbol{v}^{2}\right] \\
& +\left(348 \delta-174 \kappa_{-}+174 \delta \kappa_{+}\right)(n S)(n v)(v \Sigma)+\left(6-174 \delta \kappa_{-}+\kappa_{+}(174-348 \nu)-696 \nu\right)(n v)(n \Sigma)(v \Sigma) \\
& +\left(-144 \delta+72 \kappa_{-}-72 \delta \kappa_{+}\right)(v S)(v \Sigma)+\left(-1+36 \delta \kappa_{-}+144 \nu+\kappa_{+}(-36+72 \nu)\right)(v \Sigma)^{2} \\
& \left.+\left[\left(-9-78 \delta \kappa_{-}+\kappa_{+}(78-156 \nu)-312 \nu\right)(n v)^{2}+\left(-3+72 \delta \kappa_{-}+288 \nu+72 \kappa_{+}(-1+2 \nu)\right) \boldsymbol{v}^{2}\right] \Sigma^{2}\right] .
\end{aligned}
$$

At $3 \mathrm{PN}$ order, we have

$$
\left(\frac{d E}{d t}\right)_{\mathrm{NLO}-\mathrm{SS}}=\frac{G^{3} m^{2} \nu^{2}}{105 r^{6}}\left[f_{6}^{0}+\frac{G m}{r} f_{6}^{1}+\frac{G^{2} m^{2}}{r^{2}} f_{6}^{2}\right],
$$

with the value of the $f_{6}^{0}, f_{6}^{1}$, and $f_{6}^{2}$ coefficients given in Appendix C. After using the transformation in (17), we have checked that the above expression is equivalent to the one obtained in [95].

\section{Nonprecessing (quasi)circular orbits}

We consider here the case of aligned-spin (quasi)circular orbits. Since radiation-reaction effects first enter at $2.5 \mathrm{PN}$ order beyond the leading effects, namely at $4 \mathrm{PN}$ and $4.5 \mathrm{PN}$ for spin-orbit and spin-spin contributions, respectively, we can safely ignore them here while working to $3 \mathrm{PN}$ order. Hence, we can consistently replace $\dot{r}=0$ and $v^{2}=r^{2} \omega^{2}$ in (36) and (37) to obtain the emitted radiation. However, we must still face a radiation-reaction force which may induce the precession of the orbital plane. Even though the precession effects might be small, $d \ell / d t \leq \mathcal{O}\left(v^{8}\right)$, these may accumulate over time. Nonadiabatic methods, such as dynamical renormalization group, offer an alternative approach that remains valid over a longer timescale by resumming secular effects $[113,114]$. In this work, however, we restrict our results to timescales shorter than those induced by the secular evolution of the orbital plane. We find

$$
\begin{aligned}
\left(\frac{d E}{d t}\right)_{\mathrm{SS}}= & -\frac{32 x^{7} \nu^{2}}{5 G^{3} m^{4}}\left[\left(4+2 \kappa_{+}\right) S^{2}+\left(4 \delta-2 \kappa_{-}+2 \delta \kappa_{+}\right)(S \Sigma)+\left(\frac{1}{16}-\delta \kappa_{-}+\kappa_{+}+\left(-4-2 \kappa_{+}\right) \nu\right) \Sigma^{2}\right] \\
& -\frac{32 x^{8} \nu^{2}}{5 G^{3} m^{4}}\left[\left(-\frac{6247}{504}+\frac{57}{16} \delta \kappa_{-}-\frac{383}{112} \kappa_{+}+\left(-\frac{35}{2}-\frac{35}{4} \kappa_{+}\right) \nu\right) S^{2}\right. \\
& +\left(-\frac{1865}{112} \delta+\frac{391}{56} \kappa_{-}-\frac{391}{56} \delta \kappa_{+}+\left(-\frac{35}{2} \delta-\frac{11}{2} \kappa_{-}-\frac{35}{4} \delta \kappa_{+}\right) \nu\right)(S \Sigma) \\
& \left.+\left(-\frac{51}{16}+\frac{391}{112} \delta \kappa_{-}-\frac{391}{112} \kappa_{+}+\left(\frac{6239}{336}+\frac{13}{16} \delta \kappa_{-}+\frac{691}{112} \kappa_{+}\right) \nu+\left(\frac{35}{2}+\frac{35}{4} \kappa_{+}\right) \nu^{2}\right) \Sigma^{2}\right]
\end{aligned}
$$

or, in terms of the conserved-norm spin vectors,

$$
\begin{aligned}
\left(\frac{d E}{d t}\right)_{\mathrm{SS}}= & -\frac{32 x^{7} \nu^{2}}{5 G^{3} m^{4}}\left[\left(4+2 \kappa_{+}\right) S_{c}^{2}+\left(4 \delta-2 \kappa_{-}+2 \delta \kappa_{+}\right)\left(S_{c} \Sigma_{c}\right)+\left(\frac{1}{16}-\delta \kappa_{-}+\kappa_{+}+\left(-4-2 \kappa_{+}\right) \nu\right) \boldsymbol{\Sigma}_{c}^{2}\right] \\
& -\frac{32 x^{8} \nu^{2}}{5 G^{3} m^{4}}\left[\left(-\frac{5239}{504}+\frac{41}{16} \delta \kappa_{-}-\frac{271}{112} \kappa_{+}+\left(-\frac{43}{2}-\frac{43}{4} \kappa_{+}\right) \nu\right) S_{c}^{2}\right. \\
& +\left(-\frac{817}{56} \delta+\frac{279}{56} \kappa_{-}-\frac{279}{56} \delta \kappa_{+}+\left(-\frac{43}{2} \delta+\frac{1}{2} \kappa_{-}-\frac{43}{4} \delta \kappa_{+}\right) \nu\right)\left(S_{c} \Sigma_{c}\right) \\
& \left.+\left(-\frac{25}{8}+\frac{279}{112} \delta \kappa_{-}-\frac{279}{112} \kappa_{+}+\left(\frac{344}{21}+\frac{45}{16} \delta \kappa_{-}+\frac{243}{112} \kappa_{+}\right) \nu+\left(\frac{43}{2}+\frac{43}{4} \kappa_{+}\right) \nu^{2}\right) \boldsymbol{\Sigma}_{c}^{2}\right] .
\end{aligned}
$$




\section{B. Angular momentum}

The source multipoles allow us also to compute the radiated angular momentum via

$$
\begin{aligned}
\frac{d \boldsymbol{J}^{i}}{d t}= & -G \epsilon^{i a b}\left(\frac{2}{5} I_{a j}^{(2)} I_{b j}^{(3)}+\frac{32}{45} J_{a j}^{(2)} J_{b j}^{(3)}+\frac{1}{63} I_{a j k}^{(3)} I_{b j k}^{(4)}\right. \\
& \left.+\frac{1}{28} J_{a j k}^{(3)} J_{b j k}^{(4)}+\cdots\right),
\end{aligned}
$$

which may be also expanded into PN contributions as

$$
\begin{aligned}
\frac{d \boldsymbol{J}^{i}}{d t}= & \left(\frac{d \boldsymbol{J}^{i}}{d t}\right)_{\mathrm{N}}+\left(\frac{d \boldsymbol{J}^{i}}{d t}\right)_{\mathrm{1PN}}+\left(\frac{d \boldsymbol{J}^{i}}{d t}\right)_{\mathrm{LO}-\mathrm{SO}} \\
& +\left[\left(\frac{d \boldsymbol{J}^{i}}{d t}\right)_{2 \mathrm{PN}}+\left(\frac{d \boldsymbol{J}^{i}}{d t}\right)_{\mathrm{LO}-\mathrm{SS}}\right]+\left(\frac{d \boldsymbol{J}^{i}}{d t}\right)_{\mathrm{NLO}-\mathrm{SO}} \\
& +\left[\left(\frac{d \boldsymbol{J}^{i}}{d t}\right)_{3 \mathrm{PN}}+\left(\frac{d \boldsymbol{J}^{i}}{d t}\right)_{\mathrm{NLO}-\mathrm{SS}}\right]+\cdots
\end{aligned}
$$

For completeness, we include here both the spin-orbit and spin-spin terms to NLO in the PN expansion. The results can be written as

$$
\begin{gathered}
\left(\frac{d \boldsymbol{J}^{i}}{d t}\right)_{\text {LO-SO }}=-\frac{G^{2} m^{2} \nu^{2}}{15 r^{3}}\left[g_{3}^{0 i}+4 \frac{G m}{r} g_{3}^{1 i}+8 \frac{G^{2} m^{2}}{r^{2}} g_{3}^{2 i}\right] \\
\left(\frac{d \boldsymbol{J}^{i}}{d t}\right)_{\text {LO-SS }}=-\frac{G^{2} m \nu^{2}}{5 r^{4}}\left[g_{4}^{0 i}+\frac{G m}{r} g_{4}^{1 i}\right], \\
\left(\frac{d \boldsymbol{J}^{i}}{d t}\right)_{\mathrm{NLO}-\mathrm{SO}} \\
=-\frac{G^{2} m^{2} \nu^{2}}{105 r^{3}}\left[g_{5}^{0 i}+\frac{G m}{r} g_{5}^{1 i}+\frac{G^{2} m^{2}}{r^{2}} g_{5}^{2 i}+\frac{G^{3} m^{3}}{r^{3}} g_{5}^{3 i}\right], \\
\left(\frac{d \boldsymbol{J}^{i}}{d t}\right)_{\mathrm{NLO}-\mathrm{SS}}=-\frac{G^{2} m \nu^{2}}{35 r^{4}}\left[\frac{1}{2} g_{6}^{0 i}+\frac{G m}{r} \frac{1}{3} g_{6}^{1 i}+\frac{G^{2} m^{2}}{r^{2}} \frac{1}{3} g_{6}^{2 i}\right]
\end{gathered}
$$

with the $g_{3}^{(0,1,2) i}, g_{4}^{(0,1) i}, g_{5}^{(0,1,2,3) i}$, and $g_{6}^{(0,1,2) i}$ coefficients displayed in Appendix C. The leading order spin-orbit and spin-spin expressions agree with corresponding results in [100,111].

As a nontrivial check, we have verified that the relationship

$$
\frac{d J^{i}}{d t}=\frac{1}{\omega} \frac{d E}{d t} \boldsymbol{\ell}^{i},
$$

holds for spin effects to 3PN order for nonprecessing (quasi)circular orbits.

\section{Linear momentum and center of mass}

Finally, we can compute the flux associated with linear momentum and CM of the binary systems, which evolve due to the emission of GWs according to, e.g., [115],

$$
\begin{aligned}
\frac{d \boldsymbol{P}^{i}}{d t}= & -G\left(\frac{2}{63} I_{i j k}^{(4)} I_{j k}^{(3)}+\frac{16}{45} \epsilon_{i j k} I_{j l}^{(3)} J_{k l}^{(3)}+\frac{1}{126} \epsilon_{i j k} I_{j l m}^{(4)} J_{k l m}^{(4)}\right. \\
& \left.+\frac{4}{63} J_{i j k}^{(4)} J_{j k}^{(3)}+\cdots\right)
\end{aligned}
$$

and (with $\boldsymbol{G}^{i} \equiv m \boldsymbol{X}^{i}$ )

$$
\begin{aligned}
\frac{d \boldsymbol{G}^{i}}{d t}= & \boldsymbol{P}^{i}-G\left(\frac{1}{21}\left(I_{i j k}^{(3)} I_{j k}^{(3)}-I_{i j k}^{(4)} I_{j k}^{(2)}\right)\right. \\
& \left.+\frac{2}{21}\left(J_{i j k}^{(3)} J_{j k}^{(3)}-J_{i j k}^{(4)} J_{j k}^{(2)}\right)+\cdots\right),
\end{aligned}
$$

respectively. We can once again expand in various PN contributions,

$$
\begin{aligned}
\frac{d \boldsymbol{P}^{i}}{d t}= & \left(\frac{d \boldsymbol{P}^{i}}{d t}\right)_{1 \mathrm{PN}}+\left(\frac{d \boldsymbol{P}^{i}}{d t}\right)_{\text {LO-SO }} \\
& +\left(\frac{d \boldsymbol{P}^{i}}{d t}\right)_{2 \mathrm{PN}}+\left(\frac{d \boldsymbol{P}^{i}}{d t}\right)_{\text {NLO-SO }} \\
& +\left[\left(\frac{d \boldsymbol{P}^{i}}{d t}\right)_{3 \mathrm{PN}}+\left(\frac{d \boldsymbol{P}^{i}}{d t}\right)_{\text {LO-SS }}\right]+\cdots,
\end{aligned}
$$

and likewise,

$$
\begin{aligned}
\frac{d \boldsymbol{G}^{i}}{d t}= & \left(\frac{d \boldsymbol{G}^{i}}{d t}\right)_{1 \mathrm{PN}}+\left(\frac{d \boldsymbol{G}^{i}}{d t}\right)_{2 \mathrm{PN}}+\left(\frac{d \boldsymbol{G}^{i}}{d t}\right)_{\text {LO-SO }} \\
& +\left[\left(\frac{d \boldsymbol{G}^{i}}{d t}\right)_{3 \mathrm{PN}}+\left(\frac{d \boldsymbol{G}^{i}}{d t}\right)_{\text {LO-SS }}\right]+\cdots .
\end{aligned}
$$

Unfortunately, we do not have all the necessary source multipoles to complete the spin corrections to NLO in the $\mathrm{CM}$, notably missing the NLO corrections to $J_{i j k}$, and therefore for the purpose of this paper we will keep spinspin effects at leading order. We do, however, have all the information to compute the NLO spin-orbit contributions to the linear momentum. Inputing the source multipoles we find, in the CM frame, ${ }^{9}$

\footnotetext{
${ }^{9}$ Technically speaking, the condition $\boldsymbol{X}=0$ for the CM frame becomes more subtle once we allow for noninertial motion, due to $\mathrm{GW}$ emission. However, these effects can be ignored to 3PN order thanks to its nonsecular behavior, i.e., $\boldsymbol{X}=\mathcal{O}\left(x^{7 / 2}\right)$, see, e.g., [116].
} 


$$
\begin{gathered}
\left(\frac{d \boldsymbol{P}^{i}}{d t}\right)_{1 \mathrm{PN}}=-\frac{8 \delta G^{3} m^{4} \nu^{2}}{105 r^{4}}\left[\boldsymbol{v}^{i}\left(-\frac{8 G m}{r}+38(n v)^{2}-50 \boldsymbol{v}^{2}\right)+\boldsymbol{n}^{i}(n v)\left(\frac{12 G m}{r}-45(n v)^{2}+55 \boldsymbol{v}^{2}\right)\right], \\
\left(\frac{d \boldsymbol{P}^{i}}{d t}\right)_{\text {LO-SO }}=-\frac{8 G^{3} m^{3} \nu^{2}}{15 r^{5}}\left[4(n v)(\boldsymbol{v} \times \boldsymbol{\Sigma})^{i}-2(\boldsymbol{n} \times \boldsymbol{\Sigma})^{i} \boldsymbol{v}^{2}+(\boldsymbol{n} \times \boldsymbol{v})^{i}(-3(n v)(n \Sigma)-2(v \Sigma))\right], \\
\left(\frac{d \boldsymbol{P}^{i}}{d t}\right)_{\text {NLO-SO }}=-\frac{4 G^{3} m^{3} \nu^{2}}{945 r^{5}}\left[h_{5}^{0 i}+\frac{G m}{r} h_{5}^{1 i}+\frac{G^{2} m^{2}}{r^{2}} h_{5}^{2 i}\right], \\
\left(\frac{d \mathbf{P}^{i}}{d t}\right)_{\text {LO-SS }}=-\frac{2 G^{3} m^{2} \nu^{2}}{105 r^{6}}\left[h_{6}^{0 i}+4 \frac{G m}{r} h_{6}^{1 i}\right],
\end{gathered}
$$

whereas for the $\mathrm{CM}$ position we have

$$
\begin{gathered}
\left(\frac{d \boldsymbol{G}^{i}}{d t}\right)_{1 \mathrm{PN}}=-\boldsymbol{v}^{i}\left(\frac{24 \delta G^{3} m^{4} \nu^{2}(n v)}{35 r^{3}}+\frac{4 \delta G^{2} m^{3} \nu^{2}(n v)\left(15(n v)^{2}-29 \boldsymbol{v}^{2}\right)}{105 r^{2}}\right)-\boldsymbol{n}^{i}\left(-\frac{16 \delta G^{4} m^{5} \nu^{2}}{35 r^{4}}+\frac{4 \delta G^{3} m^{4} \nu^{2}\left(89(n v)^{2}-101 \boldsymbol{v}^{2}\right)}{105 r^{3}}\right. \\
\left.-\frac{2 \delta G^{2} m^{3} \nu^{2}\left(225(n v)^{4}-366(n v)^{2} \boldsymbol{v}^{2}+113\left(\boldsymbol{v}^{2}\right)^{2}\right)}{105 r^{2}}\right) \\
\left(\frac{d \boldsymbol{G}^{i}}{d t}\right)_{\text {LO-SO }}=-\frac{G^{2} m^{2} \nu^{2}}{105 r^{3}}\left[k_{5}^{0 i}+2 \frac{G m}{r} k_{5}^{1 i}+8 \frac{G^{2} m^{2}}{r^{2}} k_{5}^{2 i}\right] \\
\left(\frac{d \boldsymbol{G}^{i}}{d t}\right)_{\text {LO-SS }}=-\frac{G^{2} m \nu^{2}}{105 r^{4}}\left[k_{6}^{0 i}+\frac{G m}{r} k_{6}^{1 i}+\frac{G^{2} m^{2}}{r^{2}} k_{6}^{2 i}\right]
\end{gathered}
$$

The explicit expressions for the $h_{5}^{(0,1,2) i}, h_{6}^{(0,1) i}$ and $k_{5}^{(0,1,2) i}, k_{6}^{(0,1,2) i}$ coefficients are collected in Appendix C.

The calculation of the radiated linear momentum was also computed in [100], using the earlier results in [42,91]. After transforming from the locally flat and PN frames, through the relationship [46]

$$
\overline{\boldsymbol{S}}_{A}=\left(1-\frac{\boldsymbol{v}_{A}^{2}}{2}-\frac{G m_{B}}{r}\right) \boldsymbol{S}_{A}+\left(\boldsymbol{v}_{A} \cdot \boldsymbol{S}_{A}\right) \boldsymbol{v}_{A}+\cdots
$$

we find agreement for spin-orbit contributions to NLO in the PN expansion. However, we disagree on the spin-spin corrections for generic orbits. We have explicitly checked that the difference is due to the omission of the finite-size contributions to the current quadrupole and mass octupole moments.

\section{Nonprecessing (quasi)circular orbits}

The above expressions take on a much simpler form for the case of (quasi)circular orbits. Using the conserved-norm spin vector, they become

$$
\begin{aligned}
\frac{d \boldsymbol{P}^{i}}{d t}= & -\frac{\lambda^{i} \nu^{2}}{315 G}\left\{\left(-1392 \delta x^{11 / 2}+\cdots\right)+\frac{336 x^{6}\left(\Sigma_{c} \ell\right)}{G m^{2}}+x^{7}\left(\frac{7520 \delta\left(S_{c} \ell\right)}{G m^{2}}+\frac{(1608-9888 \nu)\left(\Sigma_{c} \ell\right)}{G m^{2}}\right)\right. \\
& +x^{15 / 2}\left[\left(-\frac{6960 \delta}{G^{2} m^{4}}-\frac{240 \kappa_{-}}{G^{2} m^{4}}-\frac{3480 \delta \kappa_{+}}{G^{2} m^{4}}\right) \boldsymbol{S}_{c}^{2}\right. \\
& +\left(\frac{3240 \delta \kappa_{-}}{G^{2} m^{4}}+\frac{120 \kappa_{+}(-27+116 \nu)}{G^{2} m^{4}}+\frac{48(-181+580 \nu)}{G^{2} m^{4}}\right)\left(S_{c} \Sigma_{c}\right) \\
& \left.\left.+\left(\frac{240 \delta(-3+29 \nu)}{G^{2} m^{4}}+\frac{60 \delta \kappa_{+}(-27+58 \nu)}{G^{2} m^{4}}-\frac{60 \kappa_{-}(-27+112 \nu)}{G^{2} m^{4}}\right) \Sigma_{c}^{2}\right]\right\}+\cdots,
\end{aligned}
$$

for the linear momentum including leading spin-spin and up to NLO spin-orbit effects, whereas for the center-of-mass position we have 


$$
\begin{aligned}
\frac{d \boldsymbol{G}^{i}}{d t}= & -\frac{\boldsymbol{n}^{i} \nu^{2} m}{640}\left\{\left(-4068 \delta x^{4}+\cdots\right)+x^{11 / 2}\left(\frac{21822 \delta\left(S_{c} \ell\right)}{G m^{2}}-\frac{18(-439+1441 \nu)\left(\Sigma_{c} \ell\right)}{G m^{2}}\right)\right. \\
& +x^{6}\left[\left(-\frac{20340 \delta}{G^{2} m^{4}}+\frac{72 \kappa_{-}}{G^{2} m^{4}}-\frac{10170 \delta \kappa_{+}}{G^{2} m^{4}}\right) \boldsymbol{S}_{c}^{2}+\left(\frac{10242 \delta \kappa_{-}}{G^{2} m^{4}}-\frac{180(117-452 \nu)}{G^{2} m^{4}}+\frac{18 \kappa_{+}(-569+2260 \nu)}{G^{2} m^{4}}\right)\left(S_{c} \Sigma_{c}\right)\right. \\
& \left.\left.+\left(-\frac{180 \delta(4-113 \nu)}{G^{2} m^{4}}+\frac{9 \delta \kappa_{+}(-569+1130 \nu)}{G^{2} m^{4}}-\frac{9 \kappa_{-}(-569+2268 \nu)}{G^{2} m^{4}}\right) \boldsymbol{\Sigma}_{c}^{2}\right]\right\}+\cdots
\end{aligned}
$$

\section{Kick velocity}

An application of the above formula for the linear momentum flux is the derivation of the kick velocity, $\boldsymbol{V}_{\text {kick}}^{i}$, obtained via

$$
\boldsymbol{V}_{\mathrm{kick}}^{i}=\frac{1}{m} \int_{-\infty}^{t} d t\left(\frac{d \boldsymbol{P}^{i}}{d t}\right)
$$

which we compute taking advantage of the relation in (19a). Performing the integration with the boundary condition $x=0$ at $t=-\infty$, we arrive at

$$
\begin{aligned}
\left(\boldsymbol{V}_{\text {kick }}^{i}\right)_{\text {Spin }}= & \boldsymbol{n}^{i}\left\{-\frac{16 x^{9 / 2} \nu^{2}\left(\Sigma_{c} \ell\right)}{15 G m^{2}}+\frac{8 x^{11 / 2} \nu^{2}\left(-940 \delta\left(S_{\mathrm{c}} \ell\right)+3(-67+412 \nu)\left(\Sigma_{\mathrm{c}} \ell\right)\right)}{315 G m^{2}}\right. \\
& +x^{6}\left[\frac{8\left(2 \kappa_{-}+29 \delta\left(2+\kappa_{+}\right)\right) \nu^{2} \boldsymbol{S}_{c}^{2}}{21 G^{2} m^{4}}-\frac{8 \nu^{2}\left(-362+135 \delta \kappa_{-}+1160 \nu+5 \kappa_{+}(-27+116 \nu)\right)\left(S_{c} \Sigma_{c}\right)}{105 G^{2} m^{4}}\right. \\
& \left.\left.+\frac{4 \nu^{2}\left[\delta\left(12+\kappa_{+}(27-58 \nu)-116 \nu\right)+\kappa_{-}(-27+112 \nu)\right] \boldsymbol{\Sigma}_{c}^{2}}{21 G^{2} m^{4}}\right]\right\},
\end{aligned}
$$

where we have written the final expression in terms of the conserved-norm spin vectors. A similar result is given in [100], with full agreement in the spin-orbit sector. Yet the disagreement for spin-spin contributions remains.

\section{ACCUMULATED PHASE}

Armed with the binding energy and radiated flux it is straightforward to compute the accumulated phase. Because, the norm of the covariant spin variables are not conserved during the radiation-reaction timescale, we provide here expressions in terms of the conserved-norm spin variable. We first obtain the evolution of the orbital frequency in time over a period,

$$
\begin{aligned}
\left(\frac{\dot{\omega}}{\omega^{2}}\right)_{\mathrm{SS}}= & \frac{x^{9 / 2}}{G^{2} m^{4}}\left[\left(192+96 \kappa_{+}\right) \nu \boldsymbol{S}_{c}^{2}+\left(192 \delta-96 \kappa_{-}+96 \delta \kappa_{+}\right) \nu\left(S_{c} \Sigma_{c}\right)+\left(\left(\frac{6}{5}-48 \delta \kappa_{-}+48 \kappa_{+}\right) \nu+\left(-192-96 \kappa_{+}\right) \nu^{2}\right) \boldsymbol{\Sigma}_{c}^{2}\right] \\
& +\frac{x^{11 / 2}}{G^{2} m^{4}}\left[\left(\left(\frac{102072}{35}+\frac{886}{5} \delta \kappa_{-}+\frac{10156}{35} \kappa_{+}\right) \nu+\left(-\frac{4128}{5}-\frac{2064}{5} \kappa_{+}\right) \nu^{2}\right) \boldsymbol{S}_{c}^{2}\right. \\
& +\left(\left(\frac{14120}{7} \delta-\frac{3282}{35} \kappa_{-}-\frac{96}{5} \delta^{2} \kappa_{-}+\frac{3954}{35} \delta \kappa_{+}\right) \nu+\left(-\frac{4128}{5} \delta-\frac{1864}{5} \kappa_{-}-\frac{2064}{5} \delta \kappa_{+}\right) \nu^{2}\right)\left(S_{c} \Sigma_{c}\right) \\
& \left.+\left(\left(\frac{789}{5}-\frac{1977}{35} \delta \kappa_{-}+\frac{1977}{35} \kappa_{+}\right) \nu+\left(-\frac{47269}{35}+\frac{146}{5} \delta \kappa_{-}-\frac{4976}{35} \kappa_{+}\right) \nu^{2}+\left(\frac{4128}{5}+\frac{2064}{5} \kappa_{+}\right) \nu^{3}\right) \boldsymbol{\Sigma}_{c}^{2}\right]
\end{aligned}
$$

Hence, performing the standard integration,

$$
\phi=\int d t \omega(t)=\int d \omega \frac{\omega(t)}{\dot{\omega}}
$$

we arrive at 


$$
\begin{aligned}
(\Delta \phi)_{\mathrm{SS}} & =\frac{x^{-5 / 2}}{32 \nu G^{2} m^{4}}\left\{x^{2}\left[\left(-50-25 \kappa_{+}\right) S_{c}^{2}+\left(-50 \delta+25 \kappa_{-}-25 \delta \kappa_{+}\right)\left(S_{c} \Sigma_{c}\right)+\left(-\frac{5}{16}+\frac{25}{2} \delta \kappa_{-}-\frac{25}{2} \kappa_{+}+\left(50+25 \kappa_{+}\right) \nu\right) \Sigma_{c}^{2}\right]\right. \\
& +x^{3}\left[\left(-\frac{31075}{126}+\frac{2215}{48} \delta \kappa_{-}+\frac{15635}{84} \kappa_{+}+\left(60+30 \kappa_{+}\right) \nu\right) S_{c}^{2}\right. \\
& +\left(-\frac{9775}{42} \delta-\frac{47035}{336} \kappa_{-}+\frac{47035}{336} \delta \kappa_{+}+\left(60 \delta-\frac{2575}{12} \kappa_{-}+30 \delta \kappa_{+}\right) \nu\right)\left(S_{c} \Sigma_{c}\right) \\
& \left.\left.+\left(-\frac{410825}{2688}-\frac{47035}{672} \delta \kappa_{-}+\frac{47035}{672} \kappa_{+}+\left(\frac{23535}{112}-\frac{2935}{48} \delta \kappa_{-}-\frac{4415}{56} \kappa_{+}\right) \nu+\left(-60-30 \kappa_{+}\right) \nu^{2}\right) \Sigma_{c}^{2}\right]\right\}
\end{aligned}
$$

which agrees with the result in [95].

\section{CONCLUSIONS}

Building upon the quadratic-in-spin conservative and dissipative results obtained using the EFT approach in $[43,45,46,93,94]$ to NLO in the PN expansion, in this paper we have completed the derivation of the EOM, adiabatic invariants and associated flux-balance equations for the energy and angular momentum of binary systems with spinning compact objects. We then used these results to compute the bilinear-in-spin evolution of the orbital frequency and accumulated phase for (quasi)circular orbits to 3PN order, including finite-size effects, finding agreement with the value presented in [95]. This is not surprising since, after all, the gravitational potential computed in [95] was shown to be equivalent to the ones previously obtained in the EFT $[43,45,46]$ and Arnowitt-Deser-Misner (ADM) [74,75] approaches; and moreover, as we demonstrated here, the source multipole moments in [95] are in complete agreement with those derived before in [93], after the latter are expressed in terms of conserved-norm spin variables.

Our results here include also the angular momentum flux for the first time, which is necessary to compute the phase evolution in ellipticlike orbits, allowing us to incorporate spin effects in the waveforms for generic configurations $[101,102]$, thus extending the validity of the PN approximation towards higher frequencies. This will be crucial for proper data analysis with spinning binaries, since eccentricity can rapidly deteriorate the accuracy of waveforms relying on (quasi)circular approximations [103]. Using the source multipoles and EOM we have also computed the radiated flux of linear momentum, with which we obtained the kick velocity for (quasi)circular orbits, including linear and bilinear spin effects to NLO and leading order, respectively. While perfect agreement is found with an earlier derivation in [100] for spin-orbit effects, unfortunately we disagree in the spin-spin sector even for the case of black holes (with $C_{E S^{2}}=1$ ). We trace the discrepancy to finite-size contributions in the current quadrupole and mass octupole moments which were not included in the derivation in [100]. As it turns out, only the quadratic-in-spin correction to $J_{i k l}$ at NLO is missing to complete the value of the kick velocity at the same order. We are currently performing this calculation. Moreover, building on the recent rederivation of the spin-independent radiated fluxes at $2 \mathrm{PN}$ in [117], it is straightforward to continue pushing forward at the next order for spin effects in the EFT approach. The derivation of GW observables to $\mathrm{N}^{2} \mathrm{LO}$ is underway.

\section{ACKNOWLEDGMENTS}

The work of G. C. and R. A. P. is supported by the ERC Consolidator Grant "Precision Gravity: From the LHC to LISA," provided by the European Research Council (ERC) under the European Union's H2020 research and innovation programme, Grant No. 817791 . G. C. and R. A. P. are also supported in part by the DFG under Germany's Excellence Strategy "Quantum Universe" (Grant No. 390833306). B. P. is support in part by the National Science Foundation under Grant No. PHY-1820760. We acknowledge extensive use of the xAct packages [118].

\section{APPENDIX A: EQUATIONS OF MOTION AND SOURCE MULTIPOLES}

We summarize here the values for the spin-spin contributions to the acceleration and spin dynamics as well as the source multipole moments in the covariant SSC, first computed to NLO in $[43,45,46,49]$ and [93] within the EFT approach, respectively. As in the main text we use the shorthand notation $(A B) \equiv \boldsymbol{A} \cdot \boldsymbol{B}$ whenever $\boldsymbol{A} \neq \boldsymbol{B}$, as well as $(A B C) \equiv \boldsymbol{A} \cdot(\boldsymbol{B} \times \boldsymbol{C})$, throughout the Appendixes.

\section{Acceleration}

Spin effects in the acceleration enter at different PN orders,

$$
\begin{aligned}
\boldsymbol{a}= & \boldsymbol{a}_{\mathrm{N}}+\boldsymbol{a}_{1 \mathrm{PN}}+\boldsymbol{a}_{\mathrm{LO}-\mathrm{SO}}+\left[\boldsymbol{a}_{2 \mathrm{PN}}+\boldsymbol{a}_{\mathrm{LO}-\mathrm{SS}}\right]+\boldsymbol{a}_{\mathrm{NLO}-\mathrm{SO}} \\
& +\left[\boldsymbol{a}_{3 \mathrm{PN}}+\boldsymbol{a}_{\mathrm{NLO}-\mathrm{SS}}\right]+\cdots,
\end{aligned}
$$

where at Newtonian and 1PN order we have 


$$
\begin{gathered}
\boldsymbol{a}_{\mathrm{N}}^{i}=-\frac{G m \boldsymbol{n}^{i}}{r^{2}}, \\
\boldsymbol{a}_{1 \mathrm{PN}}^{i}=-\frac{2 G m(-2+\nu)(n v) \boldsymbol{v}^{i}}{r^{2}}+\boldsymbol{n}^{i}\left[\frac{2 G^{2} m^{2}(2+\nu)}{r^{3}}+\frac{G m\left(3 \nu\left((n v)^{2}-2 v^{2}\right)-2 v^{2}\right)}{2 r^{2}}\right] .
\end{gathered}
$$

The leading order spin-spin acceleration is given by

$$
\begin{aligned}
\boldsymbol{a}_{\mathrm{LO}-\mathrm{SS}}^{i}= & \frac{G}{m r^{4}} \frac{3}{4}\left[\left(\left(-8-4 \kappa_{+}\right)(n S)+\left(-4 \delta+2 \kappa_{-}-2 \delta \kappa_{+}\right)(n \Sigma)\right) \boldsymbol{S}^{i}\right. \\
& +\left[\left(-4 \delta+2 \kappa_{-}-2 \delta \kappa_{+}\right)(n S)+\left(2 \delta \kappa_{-}+8 \nu+\kappa_{+}(-2+4 \nu)\right)(n \Sigma)\right] \boldsymbol{\Sigma}^{i} \\
& +\boldsymbol{n}^{i}\left[\left(20+10 \kappa_{+}\right)(n S)^{2}+\left(20 \delta-10 \kappa_{-}+10 \delta \kappa_{+}\right)(n S)(n \Sigma)+\left(-5 \delta \kappa_{-}+\kappa_{+}(5-10 \nu)-20 \nu\right)(n \Sigma)^{2}\right. \\
& \left.\left.+\left(-4-2 \kappa_{+}\right) \boldsymbol{S}^{2}+\left(-4 \delta+2 \kappa_{-}-2 \delta \kappa_{+}\right)(S \Sigma)+\left(\delta \kappa_{-}+4 \nu+\kappa_{+}(-1+2 \nu)\right) \boldsymbol{\Sigma}^{2}\right]\right]
\end{aligned}
$$

while we write the NLO correction as

$$
\boldsymbol{a}_{\mathrm{NLO}-\mathrm{SS}}^{i}=\frac{G}{m r^{4}}\left[\frac{1}{8} \boldsymbol{A}_{0}^{i}+\frac{G m}{r} \frac{1}{2} \boldsymbol{A}_{1}^{i}\right]
$$

with

$$
\begin{aligned}
& \boldsymbol{A}_{0}^{i}=\boldsymbol{S}^{i}\left\{\left(72+36 \delta \kappa_{-}-36 \kappa_{+}\right)(n v)(v S)+(n S)\left[\left(-60 \delta \kappa_{-}+120 \nu+60 \kappa_{+}(1+\nu)\right)(n v)^{2}\right.\right. \\
& \left.+\left(-24 \kappa_{+}(1+4 \nu)-24(1+8 \nu)\right) v^{2}\right]+(n \Sigma)\left[\left(60 \delta(-2+\nu)+30 \delta \kappa_{+}(2+\nu)\right.\right. \\
& \left.\left.+\kappa_{-}(-60+90 \nu)\right)(n v)^{2}+\left(-12 \delta \kappa_{+}(1+4 \nu)-12 \delta(-1+8 \nu)+\kappa_{-}(12+48 \nu)\right) \boldsymbol{v}^{2}\right] \\
& \left.+\left(120 \delta-36 \delta \kappa_{+}+\kappa_{-}(36-72 \nu)\right)(n v)(v \Sigma)\right\}+\Sigma^{i}\left\{\left(48 \delta-36 \delta \kappa_{+}+\kappa_{-}(36-72 \nu)\right)(n v)(v S)\right. \\
& +(n S)\left[\left(60 \delta \nu+30 \delta \kappa_{+}(2+\nu)+\kappa_{-}(-60+90 \nu)\right)(n v)^{2}\right. \\
& \left.+\left(-12 \delta \kappa_{+}(1+4 \nu)-12 \delta(1+8 \nu)+\kappa_{-}(12+48 \nu)\right) v^{2}\right]+(n \Sigma)\left[\left(30 \delta \kappa_{-}(-2+\nu)-120(-1+\nu)^{2}\right.\right. \\
& \left.\left.-30 \kappa_{+}\left(-2+5 \nu+2 \nu^{2}\right)\right)(n v)^{2}+\left(12 \delta \kappa_{-}(1+4 \nu)+12 \kappa_{+}(-1+2 \nu)(1+4 \nu)+24\left(1-\nu+8 \nu^{2}\right)\right) v^{2}\right] \\
& \left.+\left(96-36 \delta \kappa_{-}(-1+\nu)-264 \nu+36 \kappa_{+}(-1+3 \nu)\right)(n v)(v \Sigma)\right\}+\boldsymbol{v}^{i}\left\{\left(120 \kappa_{+}(-2+\nu)+240 \nu\right)(n S)^{2}(n v)\right. \\
& +\left(-120 \kappa_{-}(-2+\nu)+120 \delta \kappa_{+}(-2+\nu)+240 \delta(1+\nu)\right)(n S)(n v)(n \Sigma) \\
& +\left(-60 \delta \kappa_{-}(-2+\nu)-60 \kappa_{+}(-2+\nu)(-1+2 \nu)-240\left(-1+2 \nu+\nu^{2}\right)\right)(n v)(n \Sigma)^{2} \\
& +\left(-12 \delta \kappa_{-}+\kappa_{+}(60-24 \nu)-48(1+\nu)\right)(n v) S^{2} \\
& +\left(-24 \delta \kappa_{+}(-3+\nu)+72 \kappa_{-}(-1+\nu)-48 \delta(3+\nu)\right)(n v)(S \Sigma) \\
& +\left(72+12 \delta \kappa_{-}+\kappa_{+}(84-24 \nu)-48 \nu\right)(n S)(v S)+\left(-12 \delta \kappa_{+}(-3+\nu)-24 \delta \nu-12 \kappa_{-}(3+\nu)\right)(n \Sigma)(v S) \\
& +\left(-12 \delta \kappa_{+}(-3+\nu)-24 \delta(-1+\nu)-12 \kappa_{-}(3+\nu)\right)(n S)(v \Sigma) \\
& +\left(-36 \delta \kappa_{-}+12 \kappa_{+}\left(3-6 \nu+2 \nu^{2}\right)+24\left(-2+\nu+2 \nu^{2}\right)\right)(n \Sigma)(v \Sigma) \\
& \left.+\left(12 \delta \kappa_{-}(-3+2 \nu)+48\left(-2+5 \nu+\nu^{2}\right)+12 \kappa_{+}\left(3-8 \nu+2 \nu^{2}\right)\right)(n v) \boldsymbol{\Sigma}^{2}\right\} \\
& +\boldsymbol{n}^{i}\left\{\left(-360-60 \delta \kappa_{-}+60 \kappa_{+}\right)(n S)(n v)(v S)+\left(-240 \delta+60 \delta \kappa_{+}+60 \kappa_{-}(-1+2 \nu)\right)(n v)(n \Sigma)(v S)\right. \\
& +\left(12 \delta \kappa_{-}+12 \kappa_{+}(-1+\nu)+24(3+\nu)\right)(v S)^{2}+(n S)(n \Sigma)\left[\left(-420 \delta \nu+210 \kappa_{-} \nu-210 \delta \kappa_{+} \nu\right)(n v)^{2}\right. \\
& \left.+\left(-360 \kappa_{-} \nu+240 \delta \kappa_{+} \nu+60 \delta(1+8 \nu)\right) v^{2}\right]+(n S)^{2}\left[\left(-420 \nu-210 \kappa_{+} \nu\right)(n v)^{2}+\left(30 \delta \kappa_{-}+60(1+8 \nu)\right.\right. \\
& \left.\left.+30 \kappa_{+}(1+8 \nu)\right) \boldsymbol{v}^{2}\right]+(n \Sigma)^{2}\left[\left(105 \delta \kappa_{-} \nu+420 \nu^{2}+105 \kappa_{+} \nu(-1+2 \nu)\right)(n v)^{2}\right. \\
& \left.+\left(-150 \delta \kappa_{-} \nu-30 \kappa_{+} \nu(-5+8 \nu)-60 \nu(1+8 \nu)\right) v^{2}\right]+S^{2}\left[\left(30 \delta \kappa_{-}+30 \kappa_{+}(-1+\nu)+60(2+\nu)\right)(n v)^{2}\right. \\
& \left.+\left(-12 \delta \kappa_{-}-48 \kappa_{+} \nu-12(3+8 \nu)\right) v^{2}\right]+(S \Sigma)\left[\left(\kappa_{-}(60-150 \nu)+30 \delta \kappa_{+}(-2+\nu)+60 \delta(4+\nu)\right)(n v)^{2}\right. \\
& \left.+\left(12 \delta^{3} \kappa_{+}-12 \delta(5+8 \nu)+\kappa_{-}(-12+96 \nu)\right) v^{2}\right]+\left(-360 \delta+60 \delta \kappa_{+}+60 \kappa_{-}(-1+2 \nu)\right)(n S)(n v)(v \Sigma)
\end{aligned}
$$




$$
\begin{aligned}
& +\left(\kappa_{+}(60-180 \nu)+60 \delta \kappa_{-}(-1+\nu)+120(-2+7 \nu)\right)(n v)(n \Sigma)(v \Sigma) \\
& +\left(\kappa_{-}(24-60 \nu)+12 \delta \kappa_{+}(-2+\nu)+24 \delta(5+\nu)\right)(v S)(v \Sigma) \\
& +\left(-6 \delta \kappa_{-}(-2+3 \nu)-24\left(-2+7 \nu+\nu^{2}\right)-6 \kappa_{+}\left(2-7 \nu+2 \nu^{2}\right)\right)(v \Sigma)^{2} \\
& +\left[\left(-15 \delta \kappa_{-}(-2+3 \nu)-60\left(-2+6 \nu+\nu^{2}\right)-15 \kappa_{+}\left(2-7 \nu+2 \nu^{2}\right)\right)(n v)^{2}\right. \\
& \left.\left.+\left(6 \delta \kappa_{-}(-1+6 \nu)+6 \kappa_{+}\left(1-8 \nu+8 \nu^{2}\right)+12\left(-2+7 \nu+8 \nu^{2}\right)\right) \nu^{2}\right] \Sigma^{2}\right\}
\end{aligned}
$$

$$
\begin{aligned}
A_{1}^{i}= & S^{i}\left[\left(82-12 \delta \kappa_{-}+36 \nu+18 \kappa_{+}(2+\nu)\right)(n S)+\left(18 \delta(2+\nu)+3 \delta \kappa_{+}(8+3 \nu)+3 \kappa_{-}(-8+5 \nu)\right)(n \Sigma)\right] \\
& +\boldsymbol{\Sigma}^{i}\left[\left(6 \delta(7+3 \nu)+3 \delta \kappa_{+}(8+3 \nu)+3 \kappa_{-}(-8+5 \nu)\right)(n S)\right. \\
& \left.+\left(3 \delta \kappa_{-}(-8+\nu)-2 \nu(37+18 \nu)-3 \kappa_{+}\left(-8+17 \nu+6 \nu^{2}\right)\right)(n \Sigma)\right] \\
& +\boldsymbol{n}^{i}\left[\left(24 \delta \kappa_{-}-48 \kappa_{+}(2+\nu)-6(35+16 \nu)\right)(n S)^{2}\right. \\
& +\left(\kappa_{-}(120-48 \nu)-24 \delta \kappa_{+}(5+2 \nu)-6 \delta(33+16 \nu)\right)(n S)(n \Sigma) \\
& +\left(60 \delta \kappa_{-}+6 \nu(31+16 \nu)+12 \kappa_{+}\left(-5+10 \nu+4 \nu^{2}\right)\right)(n \Sigma)^{2}+\left(-4 \delta \kappa_{-}+10 \kappa_{+}(2+\nu)+4(9+5 \nu)\right) S^{2} \\
& +\left(6 \kappa_{-}(-4+\nu)+4 \delta(9+5 \nu)+2 \delta \kappa_{+}(12+5 \nu)\right)(S \Sigma) \\
& \left.+\left(-\delta \kappa_{-}(12+\nu)-4 \nu(9+5 \nu)+\kappa_{+}\left(12-23 \nu-10 \nu^{2}\right)\right) \Sigma^{2}\right] .
\end{aligned}
$$

\section{Spin dynamics}

In terms of the covariant spin vector, without yet transforming to a conserved-norm spin, the evolution equation is given by

$$
\begin{aligned}
\frac{d \boldsymbol{S}_{1}^{i}}{d t}= & {\left[-\frac{G m_{2}^{2}(n v) \boldsymbol{S}_{1}^{i}}{m r^{2}}+\frac{2 G m_{1} m_{2}\left(n S_{1}\right) \boldsymbol{v}^{i}}{m r^{2}}+\frac{2 G m_{2}^{2}\left(n S_{1}\right) \boldsymbol{v}^{i}}{m r^{2}}-\frac{2 G m_{1} m_{2} \boldsymbol{n}^{i}\left(v S_{1}\right)}{m r^{2}}-\frac{G m_{2}^{2} \boldsymbol{n}^{i}\left(v S_{1}\right)}{m r^{2}}\right]_{\mathrm{LOSO}} } \\
& +\left[\frac{3 C_{E S^{2}}^{(1)} G m_{2}\left(\boldsymbol{n} \times \boldsymbol{S}_{1}\right)^{i}\left(n S_{1}\right)}{m_{1} r^{3}}+\frac{3 G\left(\boldsymbol{n} \times \boldsymbol{S}_{1}\right)^{i}\left(n S_{2}\right)}{r^{3}}+\frac{G\left(\boldsymbol{S}_{1} \times \boldsymbol{S}_{2}\right)^{i}}{r^{3}}\right]_{\mathrm{LOSS}} \\
& +\left[-\frac{\delta G^{2} m m_{2} \nu \boldsymbol{n}^{i}\left(n S_{1}\right)(n v)}{2 r^{3}}+\frac{G^{2} m_{1} m_{2}^{2}(n v) \boldsymbol{S}_{1}^{i}}{m r^{3}}+\frac{\delta G^{2} m m_{2} \nu(n v) \boldsymbol{S}_{1}^{i}}{r^{3}}+\frac{3 G m_{1}^{2} m_{2}^{2}(n v)^{3} \boldsymbol{S}_{1}^{i}}{2 m^{3} r^{2}}\right. \\
& -\frac{G^{2} m_{1}^{2} m_{2}\left(n S_{1}\right) \boldsymbol{v}^{i}}{m r^{3}}-\frac{3 G^{2} m_{1} m_{2}^{2}\left(n S_{1}\right) \boldsymbol{v}^{i}}{2 m r^{3}}-\frac{G^{2} m_{2}^{3}\left(n S_{1}\right) \boldsymbol{v}^{i}}{2 m r^{3}}-\frac{3 G m_{1}^{3} m_{2}\left(n S_{1}\right)(n v)^{2} \boldsymbol{v}^{i}}{m^{3} r^{2}} \\
& -\frac{3 G m_{1}^{2} m_{2}^{2}\left(n S_{1}\right)(n v)^{2} \boldsymbol{v}^{i}}{m^{3} r^{2}}-\frac{G m_{1}^{2} m_{2}^{2}(n v) \boldsymbol{S}_{1}^{i} \boldsymbol{v}^{2}}{m^{3} r^{2}}-\frac{G m_{1} m_{2}^{3}(n v) \boldsymbol{S}_{1}^{i} \boldsymbol{v}^{2}}{m^{3} r^{2}}-\frac{\delta G m_{2} \nu(n v) \boldsymbol{S}_{1}^{i} \boldsymbol{v}^{2}}{2 r^{2}} \\
& +\frac{2 G m_{1}^{3} m_{2}\left(n S_{1}\right) \boldsymbol{v}^{i} \boldsymbol{v}^{2}}{m^{3} r^{2}}+\frac{4 G m_{1}^{2} m_{2}^{2}\left(n S_{1}\right) \boldsymbol{v}^{i} \boldsymbol{v}^{2}}{m^{3} r^{2}}+\frac{2 G m_{1} m_{2}^{3}\left(n S_{1}\right) \boldsymbol{v}^{i} \boldsymbol{v}^{2}}{m^{3} r^{2}}+\frac{G^{2} m_{1}^{2} m_{2} \boldsymbol{n}^{i}\left(v S_{1}\right)}{m r^{3}} \\
& +\frac{G^{2} m_{1} m_{2}^{2} \boldsymbol{n}^{i}\left(v S_{1}\right)}{2 m^{3}}+\frac{G^{2} m_{2}^{3} \boldsymbol{n}^{i}\left(v S_{1}\right)}{2 m r^{3}}-\frac{\delta G^{2} m m_{2} \nu \boldsymbol{n}^{i}\left(v S_{1}\right)}{2 r^{3}}+\frac{3 G m_{1}^{3} m_{2} \boldsymbol{n}^{i}(n v)^{2}\left(v S_{1}\right)}{m^{3} r^{2}} \\
& +\frac{3 G m_{1}^{2} m_{2}^{2} \boldsymbol{n}^{i}(n v)^{2}\left(v S_{1}\right)}{2 m^{3} r^{2}}-\frac{G m_{1}^{2} m_{2}^{2}(n v) \boldsymbol{v}^{i}\left(v S_{1}\right)}{m^{3} r^{2}}-\frac{G m_{1} m_{2}^{3}(n v) \boldsymbol{v}^{i}\left(v S_{1}\right)}{m^{3} r^{2}}-\frac{2 G m_{1}^{3} m_{2} \boldsymbol{n}^{i} \boldsymbol{v}^{2}\left(v S_{1}\right)}{m^{3} r^{2}} \\
& -\frac{2 G m_{1}^{2} m_{2}^{2} \boldsymbol{n}^{i} \boldsymbol{v}^{2}\left(v S_{1}\right)}{\mathrm{m} r^{2}}+\frac{\delta m_{2} \nu \boldsymbol{n}^{i} \boldsymbol{v}^{2}\left(v S_{1}\right)}{2 r^{2}}
\end{aligned}
$$




$$
\begin{aligned}
& +\left[-\frac{G^{2} m_{2} \boldsymbol{n}^{i}\left(n S_{1} S_{2}\right)}{r^{4}}+\frac{G^{2} m_{1} \nu \boldsymbol{n}^{i}\left(n S_{1} S_{2}\right)}{r^{4}}+\frac{G^{2} m_{2} \nu \boldsymbol{n}^{i}\left(n S_{1} S_{2}\right)}{r^{4}}-\frac{2 G^{2} m_{2}\left(\boldsymbol{n} \times \boldsymbol{S}_{1}\right)^{i}\left(n S_{1}\right)}{r^{4}}\right. \\
& -\frac{3 C_{E S^{2}}^{(1)} G^{2} m_{2}\left(\boldsymbol{n} \times \boldsymbol{S}_{1}\right)^{i}\left(n S_{1}\right)}{r^{4}}-\frac{12 C_{E S^{2}}^{(1)} G^{2} m_{2}^{2}\left(\boldsymbol{n} \times \boldsymbol{S}_{1}\right)^{i}\left(n S_{1}\right)}{m_{1} r^{4}}-\frac{9 G^{2} m\left(\boldsymbol{n} \times \boldsymbol{S}_{1}\right)^{i}\left(n S_{2}\right)}{r^{4}} \\
& +\frac{G^{2} m_{2}\left(\boldsymbol{n} \times \boldsymbol{S}_{1}\right)^{i}\left(n S_{2}\right)}{r^{4}}+\frac{15 C_{E S^{2}}^{(1)} G m_{2}^{2}\left(\boldsymbol{n} \times \boldsymbol{S}_{1}\right)^{i}\left(n S_{1}\right)(n v)^{2}}{2 m^{2} r^{3}}+\frac{15 G m_{1} m_{2}\left(\boldsymbol{n} \times \boldsymbol{S}_{1}\right)^{i}\left(n S_{2}\right)(n v)^{2}}{2 m^{2} r^{3}} \\
& -\frac{3 G^{2} m\left(\boldsymbol{S}_{1} \times \boldsymbol{S}_{2}\right)^{i}}{r^{4}}+\frac{G^{2} m_{2}\left(\boldsymbol{S}_{1} \times \boldsymbol{S}_{2}\right)^{i}}{r^{4}}-\frac{3 G m_{1}^{2}(n v)^{2}\left(\boldsymbol{S}_{1} \times \boldsymbol{S}_{2}\right)^{i}}{m^{2} r^{3}}-\frac{3 G m_{1} m_{2}(n v)^{2}\left(\boldsymbol{S}_{1} \times \boldsymbol{S}_{2}\right)^{i}}{2 m^{2} r^{3}} \\
& +\frac{3 G m_{2}^{2}(n v) \boldsymbol{S}_{1}^{i}\left(S_{1} n v\right)}{m m_{1} r^{3}}+\frac{3 G m_{1} m_{2}(n v) \boldsymbol{S}_{1}^{i}\left(S_{2} n v\right)}{m^{2} r^{3}}+\frac{3 G m_{2}^{2}(n v) \boldsymbol{S}_{1}^{i}\left(S_{2} n v\right)}{m^{2} r^{3}} \\
& +\frac{3 G m_{1} m_{2}\left(n S_{1} S_{2}\right)(n v) \boldsymbol{v}^{i}}{m^{2} r^{3}}+\frac{3 C_{E S^{2}}^{(1)} G m_{2}^{3}\left(n S_{1}\right)\left(S_{1} n v\right) \boldsymbol{v}^{i}}{m^{2} m_{1} r^{3}}-\frac{6 G m_{1} m_{2}\left(n S_{1}\right)\left(S_{2} n v\right) \boldsymbol{v}^{i}}{m^{2} r^{3}}-\frac{3 G m_{2}^{2}\left(n S_{1}\right)\left(S_{2} n v\right) \boldsymbol{v}^{i}}{m^{2} r^{3}} \\
& +\frac{9 C_{E S^{2}}^{(1)} G m_{1} m_{2}\left(\boldsymbol{n} \times \boldsymbol{S}_{1}\right)^{i}\left(n S_{1}\right) \boldsymbol{v}^{2}}{2 m^{2} r^{3}}+\frac{21 C_{E S^{2}}^{(1)} G m_{2}^{2}\left(\boldsymbol{n} \times \boldsymbol{S}_{1}\right)^{i}\left(n S_{1}\right) \boldsymbol{v}^{2}}{2 m^{2} r^{3}}+\frac{9 C_{E S^{2}}^{(1)} G m_{2}^{3}\left(\boldsymbol{n} \times \boldsymbol{S}_{1}\right)^{i}\left(n S_{1}\right) \boldsymbol{v}^{2}}{2 m^{2} m_{1} r^{3}} \\
& +\frac{3 G m_{1}^{2}\left(\boldsymbol{n} \times \boldsymbol{S}_{1}\right)^{i}\left(n S_{2}\right) \boldsymbol{v}^{2}}{m^{2} r^{3}}+\frac{9 G m_{1} m_{2}\left(\boldsymbol{n} \times \boldsymbol{S}_{1}\right)^{i}\left(n \boldsymbol{S}_{2}\right) \boldsymbol{v}^{2}}{2 m^{2} r^{3}}+\frac{2 G m_{1}^{2}\left(\boldsymbol{S}_{1} \times \boldsymbol{S}_{2}\right)^{i} \boldsymbol{v}^{2}}{m^{2} r^{3}}+\frac{5 G m_{1} m_{2}\left(\boldsymbol{S}_{1} \times \boldsymbol{S}_{2}\right)^{i} \boldsymbol{v}^{2}}{2 m^{2} r^{3}} \\
& -\frac{6 C_{E S^{2}}^{(1)} G m_{2}^{2}\left(n S_{1}\right)(n v)\left(\boldsymbol{v} \times \boldsymbol{S}_{1}\right)^{i}}{m^{2} r^{3}}-\frac{6 C_{E S^{2}}^{(1)} G m_{2}^{3}\left(n S_{1}\right)(n v)\left(\boldsymbol{v} \times \boldsymbol{S}_{1}\right)^{i}}{m^{2} m_{1} r^{3}}-\frac{3 G m_{1}^{2}\left(n S_{2}\right)(n v)\left(\boldsymbol{v} \times \boldsymbol{S}_{1}\right)^{i}}{m^{2} r^{3}} \\
& -\frac{3 G m_{1} m_{2}\left(n S_{2}\right)(n v)\left(\boldsymbol{v} \times \boldsymbol{S}_{1}\right)^{i}}{m^{2} r^{3}}-\frac{3 G m_{1} m_{2} \boldsymbol{v}^{i}\left(v S_{1} S_{2}\right)}{m^{2} r^{3}}-\frac{G m_{2}^{2} \boldsymbol{v}^{i}\left(v S_{1} S_{2}\right)}{m^{2} r^{3}}-\frac{6 C_{E S^{2}}^{(1)} G m_{2}^{2}\left(\boldsymbol{n} \times \boldsymbol{S}_{1}\right)^{i}(n v)\left(v S_{1}\right)}{m^{2} r^{3}} \\
& -\frac{3 G m_{2}^{2}\left(\boldsymbol{n} \times \boldsymbol{S}_{1}\right)^{i}(n v)\left(v S_{1}\right)}{m m_{1} r^{3}}-\frac{3 C_{E S^{2}}^{(1)} G m_{2}^{3}\left(\boldsymbol{n} \times \boldsymbol{S}_{1}\right)^{i}(n v)\left(v S_{1}\right)}{m^{2} m_{1} r^{3}}-\frac{6 G m_{2}^{2} \boldsymbol{n}^{i}\left(S_{1} n v\right)\left(v S_{1}\right)}{m m_{1} r^{3}} \\
& +\frac{3 C_{E S^{2}}^{(1)} G m_{2}^{2}\left(\boldsymbol{v} \times \boldsymbol{S}_{1}\right)^{i}\left(v S_{1}\right)}{m^{2} r^{3}}+\frac{3 G m_{2}^{2}\left(\boldsymbol{v} \times \boldsymbol{S}_{1}\right)^{i}\left(v S_{1}\right)}{m m_{1} r^{3}}+\frac{3 C_{E S^{2}}^{(1)} G m_{2}^{3}\left(\boldsymbol{v} \times \boldsymbol{S}_{1}\right)^{i}\left(v S_{1}\right)}{m^{2} m_{1} r^{3}} \\
& -\frac{3 G m_{1}^{2}\left(\boldsymbol{n} \times \boldsymbol{S}_{1}\right)^{i}(n v)\left(v S_{2}\right)}{m^{2} r^{3}}-\frac{6 G m_{1} m_{2}\left(\boldsymbol{n} \times \boldsymbol{S}_{1}\right)^{i}(n v)\left(v S_{2}\right)}{m^{2} r^{3}}+\frac{2 G m_{1}^{2}\left(\boldsymbol{v} \times \boldsymbol{S}_{1}\right)^{i}\left(v S_{2}\right)}{m^{2} r^{3}} \\
& \left.+\frac{2 G m_{1} m_{2}\left(\boldsymbol{v} \times S_{1}\right)^{i}\left(v S_{2}\right)}{m^{2} r^{3}}\right]_{\text {NLOSS }}+\cdots
\end{aligned}
$$

and similarly for particle 2 after exchanging $1 \leftrightarrow 2$.

\section{Source multipoles}

Using the results in [93] and translating to CM coordinates, without yet taking the symmetric trace-free part, we find the following expressions for the source multipoles needed to $3 \mathrm{PN}$ :

$$
I_{(2 \mathrm{PN})}^{i j}=\frac{1}{2 m}\left\{\left(-\delta \kappa_{-}-\kappa_{+}\right) \boldsymbol{S}^{i} \boldsymbol{S}^{j}+4 \kappa_{-} \nu \boldsymbol{S}^{i} \boldsymbol{\Sigma}^{j}+\left(\delta \kappa_{-} \nu-\kappa_{+} \nu\right) \boldsymbol{\Sigma}^{i} \boldsymbol{\Sigma}^{j}\right\}
$$




$$
\begin{aligned}
& I_{(3 \mathrm{PN})}^{i j}=\frac{\nu}{84 m}\left\{\boldsymbol { S } ^ { i } \boldsymbol { n } ^ { j } \left[(n S)\left(\frac{84 \delta G \kappa_{-} m}{r}-\frac{12 G m(11+23 \nu)}{r}-\frac{6 G \kappa_{+} m(25+23 \nu)}{r}\right)\right.\right. \\
& \left.+(n \Sigma)\left(-\frac{46 \delta G m(-1+3 \nu)}{r}-\frac{9 G \kappa_{-} m(-13+11 \nu)}{r}-\frac{3 \delta G \kappa_{+} m(39+23 \nu)}{r}\right)\right] \\
& +\boldsymbol{n}^{i} \boldsymbol{S}^{j}\left[(n \Sigma)\left(\frac{66 \delta G m(-1+3 \nu)}{r}+\frac{3 G \kappa_{-} m(-9+7 \nu)}{r}+\frac{9 \delta G \kappa_{+} m(3+11 \nu)}{r}\right)\right. \\
& \left.+(n S)\left(-\frac{60 \delta G \kappa_{-} m}{r}+\frac{132 G m(-1+3 \nu)}{r}+\frac{6 G \kappa_{+} m(-1+33 \nu)}{r}\right)\right] \\
& +\boldsymbol{S}^{i} \boldsymbol{S}^{j}\left(\frac{34 \delta G \kappa_{-} m}{r}+\frac{218 G \kappa_{+} m}{r}+\left(-13 \delta \kappa_{-}+13 \kappa_{+}\right) \mathbf{v}^{2}\right) \\
& +\boldsymbol{v}^{i} \boldsymbol{S}^{j}\left[\left(-24 \delta \kappa_{-}+24 \kappa_{+}\right)(v S)+\left(24 \delta \kappa_{+}+\kappa_{-}(-24+48 \nu)\right)(v \Sigma)\right] \\
& +\boldsymbol{\Sigma}^{i} \boldsymbol{n}^{j}\left[(n S)\left(-\frac{9 G \kappa_{-} m(-13+11 \nu)}{r}-\frac{3 \delta G \kappa_{+} m(39+23 \nu)}{r}-\frac{2 \delta G m(89+69 \nu)}{r}\right)\right. \\
& \left.+(n \Sigma)\left(-\frac{3 \delta G \kappa_{-} m(-39+5 \nu)}{r}+\frac{4 G m \nu(61+69 \nu)}{r}+\frac{3 G \kappa_{+} m\left(-39+83 \nu+46 \nu^{2}\right)}{r}\right)\right] \\
& +\boldsymbol{\Sigma}^{i} \boldsymbol{S}^{j}\left[\frac{92 \delta G \kappa_{+} m}{r}-\frac{4 G \kappa_{-} m(23+17 \nu)}{r}+\left(13 \delta \kappa_{+}+\kappa_{-}(-13+26 \nu)\right) \boldsymbol{v}^{2}\right] \\
& -\boldsymbol{\Sigma}^{i} \boldsymbol{v}^{j} 28 \nu(v \Sigma) \boldsymbol{n}^{i} \boldsymbol{\Sigma}^{j}\left[(n S)\left(\frac{66 \delta G m(-1+3 \nu)}{r}+\frac{3 G \kappa_{-} m(-9+7 \nu)}{r}+\frac{9 \delta G \kappa_{+} m(3+11 \nu)}{r}\right)\right. \\
& \left.+(n \Sigma)\left(-\frac{3 \delta G \kappa_{-} m(9+13 \nu)}{r}-\frac{3 G \kappa_{+} m(-1+3 \nu)(9+22 \nu)}{r}-\frac{4 G m \nu(-5+99 \nu)}{r}\right)\right] \\
& +\boldsymbol{S}^{i} \boldsymbol{\Sigma}^{j}\left[\frac{92 \delta G \kappa_{+} m}{r}-\frac{4 G \kappa_{-} m(23+17 \nu)}{r}+\left(13 \delta \kappa_{+}+\kappa_{-}(-13+26 \nu)\right) \boldsymbol{v}^{2}\right] \\
& +\boldsymbol{v}^{i} \boldsymbol{\Sigma}^{j}\left[\left(24 \delta \kappa_{+}+\kappa_{-}(-24+48 \nu)\right)(v S)+\left(\kappa_{+}(24-72 \nu)+24 \delta \kappa_{-}(-1+\nu)-140 \nu\right)(v \Sigma)\right] \\
& +\boldsymbol{\Sigma}^{i} \boldsymbol{\Sigma}^{j}\left[\frac{112 G m \nu}{r}-\frac{2 \delta G \kappa_{-} m(46+17 \nu)}{r}-\frac{2 G \kappa_{+} m(-46+75 \nu)}{r}+\left(\kappa_{+}(13-39 \nu)+13 \delta \kappa_{-}(-1+\nu)+140 \nu\right) \boldsymbol{v}^{2}\right] \\
& +\boldsymbol{v}^{i} \boldsymbol{v}^{j}\left[\left(22 \delta \kappa_{-}-22 \kappa_{+}\right) \boldsymbol{S}^{2}+\left(-44 \delta \kappa_{+}+\kappa_{-}(44-88 \nu)\right)(S \Sigma)+\left(-22 \delta \kappa_{-}(-1+\nu)+28 \nu+\kappa_{+}(-22+66 \nu)\right) \boldsymbol{\Sigma}^{2}\right] \\
& +\boldsymbol{n}^{i} \boldsymbol{n}^{j}\left[(n S)^{2}\left(\frac{42 \delta G \kappa_{-} m}{r}-\frac{24 G m(-9+20 \nu)}{r}-\frac{12 G \kappa_{+} m(-9+20 \nu)}{r}\right)\right. \\
& +(n S)(n \Sigma)\left(\frac{6 G \kappa_{-} m(-11+12 \nu)}{r}-\frac{24 \delta G m(-9+20 \nu)}{r}-\frac{6 \delta G \kappa_{+} m(-11+40 \nu)}{r}\right) \\
& +(n \Sigma)^{2}\left(\frac{24 G m \nu(-9+20 \nu)}{r}+\frac{3 \delta G \kappa_{-} m(-11+26 \nu)}{r}+\frac{3 G \kappa_{+} m\left(11-48 \nu+80 \nu^{2}\right)}{r}\right) \\
& +\left(-\frac{22 \delta G \kappa_{-} m}{r}+\frac{24 G m(-4+5 \nu)}{r}+\frac{4 G \kappa_{+} m(4+15 \nu)}{r}\right) S^{2} \\
& +\left(\frac{24 \delta G m(-4+5 \nu)}{r}+\frac{2 G \kappa_{-} m(-19+14 \nu)}{r}+\frac{2 \delta G \kappa_{+} m(19+30 \nu)}{r}\right)(S \Sigma) \\
& \left.+\left(-\frac{\delta G \kappa_{-} m(19+8 \nu)}{r}-\frac{8 G m \nu(2+15 \nu)}{r}-\frac{G \kappa_{+} m\left(-19+30 \nu+60 \nu^{2}\right)}{r}\right) \boldsymbol{\Sigma}^{2}\right] \\
& \left.+\delta^{i j}\left(\frac{112 G m \nu(n \Sigma)^{2}}{r}+140 \nu(v \Sigma)^{2}+\left(-\frac{112 G m \nu}{r}-140 \nu v^{2}\right) \Sigma^{2}\right)\right\},
\end{aligned}
$$$$
I_{(2 \mathrm{PN})}^{i j k}=\frac{3 \nu}{2 m}\left\{-2 \kappa_{-} \boldsymbol{S}^{i} \boldsymbol{S}^{j} \boldsymbol{r}^{k}+\left(-\delta \kappa_{-}+\kappa_{+}\right) \boldsymbol{\Sigma}^{i} \boldsymbol{S}^{j} \boldsymbol{r}^{k}+\left(-\delta \kappa_{-}+\kappa_{+}\right) \boldsymbol{S}^{i} \boldsymbol{\Sigma}^{j} \boldsymbol{r}^{k}+\left(\delta \kappa_{+}+\kappa_{-}(-1+2 \nu)\right) \boldsymbol{\Sigma}^{i} \boldsymbol{\Sigma}^{j} \boldsymbol{r}^{k}\right\},
$$ 


$$
\begin{aligned}
J_{(2 \mathrm{PN})}^{i j}= & \frac{\nu}{2 m}\left\{2 \kappa_{-}(\boldsymbol{v} \times \boldsymbol{S})^{i} \boldsymbol{S}^{j}+\left(\delta \kappa_{-}-\kappa_{+}\right)(\boldsymbol{v} \times \boldsymbol{\Sigma})^{i} \boldsymbol{S}^{j}+3 \boldsymbol{S}^{i}(\boldsymbol{v} \times \boldsymbol{\Sigma})^{j}+\left(\delta \kappa_{-}-\kappa_{+}\right)(\boldsymbol{v} \times \boldsymbol{S})^{i} \boldsymbol{\Sigma}^{j}\right. \\
& \left.+(\boldsymbol{v} \times \mathbf{\Sigma})^{i} \boldsymbol{\Sigma}^{j}\left(-\delta \kappa_{+}+\kappa_{-}(1-2 \nu)\right)\right\} .
\end{aligned}
$$

\section{APPENDIX B: CONSERVATIVE SECTOR}

\section{Binding energy}

The coefficients of the binding energy in the CM in (16) are given by

$$
\begin{aligned}
& e_{4}^{0}=\left(12+6 \kappa_{+}\right)(n S)^{2}+\left(12 \delta-6 \kappa_{-}+6 \delta \kappa_{+}\right)(n S)(n \Sigma)+\left(-3 \delta \kappa_{-}+\kappa_{+}(3-6 \nu)-12 \nu\right)(n \Sigma)^{2} \\
& +\left(-4-2 \kappa_{+}\right) S^{2}+\left(-4 \delta+2 \kappa_{-}-2 \delta \kappa_{+}\right)(S \Sigma)+\left(\delta \kappa_{-}+4 \nu+\kappa_{+}(-1+2 \nu)\right) \Sigma^{2}, \\
& e_{6}^{0}=\left(-72 \delta-24 \kappa_{-}+24 \delta \kappa_{+}\right)(n v)(n \Sigma)(v S)+48 \kappa_{-} \nu(n v)(n \Sigma)(v S)+\left(8 \delta \kappa_{-}+4 \kappa_{+}(-2+\nu)+8(3+\nu)\right)(v S)^{2} \\
& +\left(30 \kappa_{-}+6 \delta \kappa_{+}(-5+\nu)+12 \delta(8+\nu)\right)(n S)(n \Sigma) \boldsymbol{v}^{2}+(n S)^{2}\left[\left(-60 \nu-30 \kappa_{+} \nu\right)(n v)^{2}\right. \\
& \left.+\left(6 \delta \kappa_{-}+6 \kappa_{+}(-4+\nu)+12(4+\nu)\right) v^{2}\right]+S^{2}\left[\left(6 \delta \kappa_{-}+6 \kappa_{+}(-1+\nu)+12(2+\nu)\right)(n v)^{2}\right. \\
& \left.+\left(-4 \delta \kappa_{-}-2 \kappa_{+}(-5+\nu)-4(6+\nu)\right) \boldsymbol{v}^{2}\right]+(S \Sigma)\left[\left(\kappa_{-}(12-30 \nu)+6 \delta \kappa_{+}(-2+\nu)\right.\right. \\
& \left.+12 \delta(4+\nu))(n v)^{2}+\left(-2 \delta \kappa_{+}(-7+\nu)-4 \delta(12+\nu)+2 \kappa_{-}(-7+9 \nu)\right) \boldsymbol{\nu}^{2}\right] \\
& +(n \Sigma)^{2}\left[\left(15 \delta \kappa_{-} \nu+60 \nu^{2}+15 \kappa_{+} \nu(-1+2 \nu)\right)(n v)^{2}+\left(-3 \delta \kappa_{-}(-5+3 \nu)+\kappa_{+}\left(-15+39 \nu-6 \nu^{2}\right)\right.\right. \\
& \left.\left.-12\left(-4+12 \nu+\nu^{2}\right)\right) v^{2}\right]+\left(\kappa_{+}(24-72 \nu)+24 \delta \kappa_{-}(-1+\nu)+72(-1+3 \nu)\right)(n v)(n \Sigma)(v \Sigma) \\
& +\left(\kappa_{-}(16-36 \nu)+4 \delta \kappa_{+}(-4+\nu)+8 \delta(6+\nu)\right)(v S)(v \Sigma)+\left(-2 \delta \kappa_{-}(-4+5 \nu)+\kappa_{+}\left(-8+26 \nu-4 \nu^{2}\right)\right. \\
& \left.-8\left(-3+9 \nu+\nu^{2}\right)\right)(v \Sigma)^{2}+(n S)\left[\left(-72-24 \delta \kappa_{-}+24 \kappa_{+}\right)(n v)(v S)+(n \Sigma)\left(\left(-60 \delta \nu+30 \kappa_{-} \nu\right.\right.\right. \\
& \left.\left.\left.-30 \delta \kappa_{+} \nu\right)(n v)^{2}-30 \kappa_{-} \nu v^{2}\right)+\left(-72 \delta+24 \delta \kappa_{+}+\kappa_{-}(-24+48 \nu)\right)(n v)(v \Sigma)\right] \\
& +\left[\left(\delta \kappa_{-}(6-9 \nu)+\kappa_{+}\left(-6+21 \nu-6 \nu^{2}\right)-12\left(-2+6 \nu+\nu^{2}\right)\right)(n v)^{2}+\left(\delta \kappa_{-}(-7+5 \nu)+4\left(-6+18 \nu+\nu^{2}\right)\right.\right. \\
& \left.\left.+\kappa_{+}\left(7-19 \nu+2 \nu^{2}\right)\right) \boldsymbol{v}^{2}\right] \boldsymbol{\Sigma}^{2}, \\
& e_{6}^{1}=\left(-36+9 \delta \kappa_{-}-15 \kappa_{+}\right)(n S)^{2}+\left(-32 \delta-24 \delta \kappa_{+}+\kappa_{-}(24-36 \nu)\right)(n S)(n \Sigma) \\
& +\left(30 \nu-3 \delta \kappa_{-}(-4+3 \nu)+\kappa_{+}(-12+33 \nu)\right)(n \Sigma)^{2}+\left(8-3 \delta \kappa_{-}+5 \kappa_{+}\right) S^{2} \\
& +\left(8 \delta+8 \delta \kappa_{+}+\kappa_{-}(-8+12 \nu)\right)(S \Sigma)+\left(\kappa_{+}(4-11 \nu)-10 \nu+\delta \kappa_{-}(-4+3 \nu)\right) \Sigma^{2} .
\end{aligned}
$$

\section{Angular momentum}

The coefficients of the angular momentum in the CM in (28) and (29) are given by

$$
\begin{gathered}
\ell_{5}^{0}=\boldsymbol{S}^{i}\left((4+5 \nu)(n v)^{2}-6 v^{2}\right)+\boldsymbol{v}^{i}((-6+12 \nu)(n S)(n v)+4 \delta \nu(n v)(n \Sigma)+4(v S)+2 \delta \nu(v \Sigma)) \\
+\boldsymbol{n}^{i}\left((-2-8 \nu)(n v)(v S)+(n S)\left(-9 \nu(n v)^{2}+6 v^{2}\right)+(n \Sigma)\left(-6 \delta \nu(n v)^{2}+3 \delta \nu v^{2}\right)-6 \delta \nu(n v)(v \Sigma)\right), \\
\ell_{5}^{1}=\boldsymbol{n}^{i}((-1-4 \nu)(n S)-(\delta+3 \delta \nu)(n \Sigma))+\boldsymbol{S}^{i}(1+4 \nu)+\boldsymbol{\Sigma}^{i}(\delta+3 \delta \nu),
\end{gathered}
$$




$$
\begin{aligned}
\ell_{6}^{0}= & {\left[\left(6+\delta \kappa_{-}+\kappa_{+}(-1+2 \nu)\right)(n S)+\left(6 \delta+\kappa_{-}(1-3 \nu)+\delta \kappa_{+}(-1+\nu)-2 \nu\right)(n \Sigma)\right](\boldsymbol{v} \times \boldsymbol{S})^{i} } \\
& -6 \delta(S n \Sigma) \boldsymbol{v}^{i}+\left[\left(\kappa_{-}(1-3 \nu)+\delta \kappa_{+}(-1+\nu)+2(3 \delta+\nu)\right)(n S)\right. \\
& \left.+\left(6-16 \nu+\kappa_{-}(\delta-2 \delta \nu)+\kappa_{+}\left(-1+4 \nu-2 \nu^{2}\right)\right)(n \Sigma)\right](\boldsymbol{v} \times \boldsymbol{\Sigma})^{i} \\
& +\boldsymbol{S}^{i}\left[\left(-\delta \kappa_{-}+\kappa_{+}(1-2 \nu)+6(2+\nu)\right)(S n v)+\left(\kappa_{-}(1-3 \nu)+\delta \kappa_{+}(-1+\nu)-\nu-3 \delta(2+\nu)\right)(v n \Sigma)\right] \\
& +\boldsymbol{n}^{i}\left\{6 \delta(n v)(S n \Sigma)+(n \Sigma)\left[(3 \nu-3 \delta(6+\nu))(S n v)-6\left(-2+7 \nu+\nu^{2}\right)(v n \Sigma)\right]\right. \\
& +(n S)[-6(3+\nu)(S n v)+3(\nu+\delta(4+\nu))(v n \Sigma)]\} \\
& +(\boldsymbol{n} \times \boldsymbol{S})^{i}\left\{\left(-3 \delta \kappa_{-}+6(-3+\nu)+\kappa_{+}(3+6 \nu)\right)(n S)(n v)\right. \\
& +\left[3 \kappa_{-}(-1+\nu)+3 \delta \kappa_{+}(1+\nu)+3(\delta(-6+\nu)+\nu)\right](n v)(n \Sigma)+\left(14+2 \delta \kappa_{-}+6 \nu-2 \kappa_{+}(1+\nu)\right)(v S) \\
& \left.+\left(\kappa_{-}(2-3 \nu)-\nu-\delta \kappa_{+}(2+\nu)+3 \delta(4+\nu)\right)(v \Sigma)\right\} \\
& +(\boldsymbol{n} \times \boldsymbol{\Sigma})^{i}\left[\left(3 \delta(-6+\nu)+3 \kappa_{-}(-1+\nu)-3 \nu+3 \delta \kappa_{+}(1+\nu)\right)(n S)(n v)\right. \\
& +\left(-3 \delta \kappa_{-}+\kappa_{+}\left(3-6 \nu-6 \nu^{2}\right)-6\left(3-9 \nu+\nu^{2}\right)\right)(n v)(n \Sigma) \\
& +\left(\kappa_{-}(2-3 \nu)+\nu-\delta \kappa_{+}(2+\nu)+\delta(14+3 \nu)\right)(v S) \\
& \left.+\left(12-\delta \kappa_{-}(-2+\nu)-40 \nu-6 \nu^{2}+\kappa_{+}\left(-2+5 \nu+2 \nu^{2}\right)\right)(v \Sigma)\right] \\
& +\left[\left(-\nu+3 \delta(4+\nu)+\kappa_{-}(-1+3 \nu)+\kappa_{+}(\delta-\delta \nu)\right)(S n v)\right. \\
& \left.+\left(-6+26 \nu+6 \nu^{2}+\kappa_{-}(\delta-2 \delta \nu)+\kappa_{+}\left(-1+4 \nu-2 \nu^{2}\right)\right)(v n \Sigma)\right] \boldsymbol{\Sigma}^{i} \\
& +(\boldsymbol{n} \times \boldsymbol{v})^{i}\left[\left(24-3 \kappa_{+}(3+\nu)\right)(n S)^{2}+\left(48 \delta+3 \kappa_{-}(3+\nu)-3 \delta \kappa_{+}(3+\nu)\right)(n S)(n \Sigma)\right. \\
& +\left(24-72 \nu+\frac{3}{2} \delta \kappa_{-}(3+\nu)+\frac{3}{2} \kappa_{+}(3+\nu)(-1+2 \nu)\right)(n \Sigma)^{2}+\left(3 \kappa_{+}(1+\nu)-2(7+2 \nu)\right) S^{2} \\
& +\left(-3 \kappa_{-}(1+\nu)+3 \delta \kappa_{+}(1+\nu)-2 \delta(13+2 \nu)\right)(S \Sigma) \\
& \left.+\left(-\frac{3}{2} \delta \kappa_{-}(1+\nu)-\frac{3}{2} \kappa_{+}(1+\nu)(-1+2 \nu)+4\left(-3+10 \nu+\nu^{2}\right)\right) \boldsymbol{\Sigma}^{2}\right] .
\end{aligned}
$$

\section{APPENDIX C: RADIATION SECTOR}

\section{Energy flux}

The coefficients of the radiated energy in (37) are given by

$$
\begin{aligned}
f_{6}^{0}= & (v S)^{2}\left[\left(-8688 \delta \kappa_{-}+\kappa_{+}(11424-9720 \nu)-6(529+3240 \nu)\right)(n v)^{2}\right. \\
& \left.+\left(4462+2676 \delta \kappa_{-}+3792 \nu+\kappa_{+}(-684+1896 \nu)\right) \boldsymbol{v}^{2}\right] \\
& +(n S)(v S)\left[\left(25464 \delta \kappa_{-}+3384 \kappa_{+}(-17+12 \nu)+48(-581+1692 \nu)\right)(n v)^{3}\right. \\
& \left.+\left(4800-11832 \delta \kappa_{-}+\kappa_{+}(22200-8712 \nu)-17424 \nu\right)(n v) \boldsymbol{v}^{2}\right] \\
& +(n \Sigma)(v S)\left[\left(\kappa_{-}(41496-71232 \nu)+24 \delta \kappa_{+}(-1729+846 \nu)+12 \delta(1273+3384 \nu)\right)(n v)^{3}\right. \\
& \left.+\left(-66 \delta(161+132 \nu)-12 \delta \kappa_{+}(-1418+363 \nu)+\kappa_{-}(-17016+28020 \nu)\right)(n v) \boldsymbol{v}^{2}\right] \\
& +S^{2}\left[\left(-35436-2274 \delta \kappa_{-}+28224 \nu+6 \kappa_{+}(-2153+2352 \nu)\right)(n v)^{4}\right. \\
& +\left(49800+3684 \delta \kappa_{-}+\kappa_{+}(16452-15744 \nu)-31488 \nu\right)(n v)^{2} \boldsymbol{v}^{2} \\
& \left.+\left(-16260-1374 \delta \kappa_{-}+5136 \nu+6 \kappa_{+}(-671+428 \nu)\right)\left(\boldsymbol{v}^{2}\right)^{2}\right] \\
& +(n S)(n \Sigma)\left[\left(-60 \delta \kappa_{+}(-1725+1192 \nu)-120 \delta(-591+1192 \nu)+60 \kappa_{-}(-1725+2054 \nu)\right)(n v)^{4}\right. \\
& +\left(-2448 \kappa_{-}(-32+25 \nu)+288 \delta \kappa_{+}(-272+179 \nu)+6 \delta(-10043+17184 \nu)\right)(n v)^{2} \boldsymbol{v}^{2} \\
& \left.+\left(-12 \kappa_{-}(691+438 \nu)-12 \delta \kappa_{+}(-691+548 \nu)-24 \delta(-67+548 \nu)\right)\left(\boldsymbol{v}^{2}\right)^{2}\right]
\end{aligned}
$$




$$
\begin{aligned}
& +(n S)^{2}\left[\left(81570-12930 \delta \kappa_{-}+\kappa_{+}(90570-71520 \nu)-143040 \nu\right)(n v)^{4}\right. \\
& +\left(2412 \delta \kappa_{-}+12(-4483+8592 \nu)+\kappa_{+}(-75924+51552 \nu)\right)(n v)^{2} v^{2} \\
& \left.+\left(2958 \delta \kappa_{-}+\kappa_{+}(11250-6576 \nu)-6(85+2192 \nu)\right)\left(v^{2}\right)^{2}\right] \\
& +(S \Sigma)\left[\left(\kappa_{-}(10644-5016 \nu)+252 \delta(-201+112 \nu)+12 \delta \kappa_{+}(-887+1176 \nu)\right)(n v)^{4}\right. \\
& +\left(336 \kappa_{-}(-38+3 \nu)-96 \delta \kappa_{+}(-133+164 \nu)-6 \delta(-11269+5248 \nu)\right)(n v)^{2} v^{2} \\
& \left.+\left(12 \delta \kappa_{+}(-221+214 \nu)+2 \delta(-9377+2568 \nu)+\kappa_{-}(2652+2928 \nu)\right)\left(v^{2}\right)^{2}\right] \\
& +(n \Sigma)^{2}\left[\left(90 \delta \kappa_{-}(-575+541 \nu)+30 \kappa_{+}\left(1725-5073 \nu+2384 \nu^{2}\right)+60\left(-307-1016 \nu+2384 \nu^{2}\right)\right)(n v)^{4}\right. \\
& +\left(8238+67806 \nu-103104 \nu^{2}-36 \delta \kappa_{-}(-1088+783 \nu)-36 \kappa_{+}\left(1088-2959 \nu+1432 \nu^{2}\right)\right)(n v)^{2} v^{2} \\
& \left.+\left(6 \delta \kappa_{-}(-691+55 \nu)+6\left(-515-638 \nu+2192 \nu^{2}\right)+\kappa_{+}\left(4146-8622 \nu+6576 \nu^{2}\right)\right)\left(v^{2}\right)^{2}\right] \\
& +(v S)\left[\left(-24 \delta \kappa_{+}(-838+405 \nu)-12 \delta(1543+1620 \nu)+24 \kappa_{-}(-838+1853 \nu)\right)(n v)^{2}\right. \\
& \left.+\left(-840 \kappa_{-}(-4+15 \nu)+24 \delta \kappa_{+}(-140+79 \nu)+2 \delta(3065+1896 \nu)\right) v^{2}\right](v \Sigma) \\
& +(n S)\left[\left(\kappa_{-}(41496-71232 \nu)+24 \delta \kappa_{+}(-1729+846 \nu)+48 \delta(-233+846 \nu)\right)(n v)^{3}\right. \\
& \left.+\left(-12 \delta \kappa_{+}(-1418+363 \nu)-6 \delta(-2023+1452 \nu)+\kappa_{-}(-17016+28020 \nu)\right)(n v) v^{2}\right](v \Sigma) \\
& +(n \Sigma)\left[\left(-24 \delta \kappa_{-}(-1729+1907 \nu)-192\left(-174+193 \nu+423 \nu^{2}\right)-24 \kappa_{+}\left(1729-5365 \nu+1692 \nu^{2}\right)\right)(n v)^{3}\right. \\
& \left.+\left(12 \delta \kappa_{-}(-1418+1349 \nu)+12 \kappa_{+}\left(1418-4185 \nu+726 \nu^{2}\right)+12\left(-652+275 \nu+1452 \nu^{2}\right)\right)(n v) v^{2}\right](v \Sigma) \\
& +\left[\left(12 \delta \kappa_{-}(-838+1129 \nu)+48\left(-296+668 \nu+405 \nu^{2}\right)+12 \kappa_{+}\left(838-2805 \nu+810 \nu^{2}\right)\right)(n v)^{2}\right. \\
& \left.+\left(2200-7646 \nu-3792 \nu^{2}-24 \delta \kappa_{-}(-70+151 \nu)-24 \kappa_{+}\left(70-291 \nu+79 \nu^{2}\right)\right) v^{2}\right](v \Sigma)^{2} \\
& +\left[\left(-9714+64788 \nu-28224 \nu^{2}-6 \delta \kappa_{-}(-887+797 \nu)-6 \kappa_{+}\left(887-2571 \nu+2352 \nu^{2}\right)\right)(n v)^{4}\right. \\
& +\left(12 \delta \kappa_{-}(-532+349 \nu)+12 \kappa_{+}\left(532-1413 \nu+1312 \nu^{2}\right)+6\left(2402-14085 \nu+5248 \nu^{2}\right)\right)(n v)^{2} v^{2} \\
& \left.+\left(-3754+21194 \nu-5136 \nu^{2}+6 \delta \kappa_{-}(221+15 \nu)-6 \kappa_{+}\left(221-427 \nu+428 \nu^{2}\right)\right)\left(v^{2}\right)^{2}\right] \Sigma^{2},
\end{aligned}
$$

$$
\begin{aligned}
f_{6}^{1}= & \left(20472 \delta \kappa_{-}-24(5125+161 \nu)-12 \kappa_{+}(5338+161 \nu)\right)(n S)(n v)(v S) \\
& +\left(\kappa_{-}(42264-39978 \nu)-6 \delta \kappa_{+}(7044+161 \nu)-4 \delta(11608+483 \nu)\right)(n v)(n \Sigma)(v S) \\
& +\left(27240-4664 \delta \kappa_{-}+336 \nu+\kappa_{+}(14652+168 \nu)\right)(v S)^{2} \\
& +S^{2}\left[\left(2772 \delta \kappa_{-}+8(-6109+330 \nu)+\kappa_{+}(-23844+1320 \nu)\right)(n v)^{2}\right. \\
& \left.+\left(-2572 \delta \kappa_{-}+\kappa_{+}(21028-720 \nu)-72(-581+20 \nu)\right) v^{2}\right] \\
& +(n S)^{2}\left[\left(300528-28788 \delta \kappa_{-}+\kappa_{+}(135588-2028 \nu)-4056 \nu\right)(n v)^{2}\right. \\
& \left.+\left(-182752+12380 \delta \kappa_{-}+3984 \nu+24 \kappa_{+}(-3239+83 \nu)\right) v^{2}\right] \\
& +(S \Sigma)\left[\left(\kappa_{-}(26616-12408 \nu)+264 \delta(-209+10 \nu)+24 \delta \kappa_{+}(-1109+55 \nu)\right)(n v)^{2}\right. \\
& \left.+\left(-80 \delta \kappa_{+}(-295+9 \nu)-8 \delta(-6109+180 \nu)+16 \kappa_{-}(-1475+688 \nu)\right) v^{2}\right] \\
& +(n S)(n \Sigma)\left[\left(-12 \delta \kappa_{+}(-13698+169 \nu)-12 \delta(-23537+338 \nu)+108 \kappa_{-}(-1522+1085 \nu)\right)(n v)^{2}\right. \\
& \left.+\left(\kappa_{-}(90116-51512 \nu)+4 \delta \kappa_{+}(-22529+498 \nu)+4 \delta(-45929+996 \nu)\right) v^{2}\right] \\
& +(n \Sigma)^{2}\left[\left(6 \delta \kappa_{-}(-13698+4967 \nu)+6 \kappa_{+}\left(13698-32363 \nu+338 \nu^{2}\right)+12\left(467-22056 \nu+338 \nu^{2}\right)\right)(n v)^{2}\right. \\
& \left.+\left(-2 \delta \kappa_{-}(-22529+6688 \nu)-2 \kappa_{+}\left(22529-51746 \nu+996 \nu^{2}\right)-4\left(2425-46383 \nu+996 \nu^{2}\right)\right) v^{2}\right]
\end{aligned}
$$




$$
\begin{aligned}
+ & \left(\kappa_{-}(42264-39978 \nu)-6 \delta \kappa_{+}(7044+161 \nu)-4 \delta(12686+483 \nu)\right)(n S)(n v)(v \Sigma) \\
+ & \left(9808+69804 \nu+3864 \nu^{2}-6 \delta \kappa_{-}(-7044+3251 \nu)+6 \kappa_{+}\left(-7044+17339 \nu+322 \nu^{2}\right)\right)(n v)(n \Sigma)(v \Sigma) \\
+ & \left(336 \delta(55+\nu)+4 \delta \kappa_{+}(4829+42 \nu)+4 \kappa_{-}(-4829+4622 \nu)\right)(v S)(v \Sigma) \\
+ & \left(2 \delta \kappa_{-}(-4829+2290 \nu)+\kappa_{+}\left(9658-23896 \nu-168 \nu^{2}\right)-16\left(299+613 \nu+21 \nu^{2}\right)\right)(v \Sigma)^{2} \\
+ & {\left[\left(-12 \delta \kappa_{-}(-1109+286 \nu)-12 \kappa_{+}\left(1109-2504 \nu+110 \nu^{2}\right)-8\left(151-7717 \nu+330 \nu^{2}\right)\right)(n v)^{2}\right.} \\
+ & \left.\left(4 \delta \kappa_{-}(-2950+733 \nu)+8\left(551-7036 \nu+180 \nu^{2}\right)+4 \kappa_{+}\left(2950-6633 \nu+180 \nu^{2}\right)\right) \nu^{2}\right] \Sigma^{2}, \\
f_{6}^{2}= & \left(936+48 \delta \kappa_{-}+432 \delta^{2} \kappa_{+}-3456 \nu\right)(n S)^{2}+\left(-384 \delta^{2} \kappa_{-}-192 \delta \kappa_{+}(-2+9 \nu)-16 \delta(-49+216 \nu)\right)(n S)(n \Sigma) \\
& +\left(-48 \delta^{2} \kappa_{+}(-4+9 \nu)+48 \delta \kappa_{-}(-4+17 \nu)-16 \delta^{2}(-1+54 \nu)\right)(n \Sigma)^{2} \\
& +\left(-16 \delta \kappa_{-}-144 \delta^{2} \kappa_{+}+16(-23+72 \nu)\right) S^{2}+\left(128 \delta^{2} \kappa_{-}+64 \delta \kappa_{+}(-2+9 \nu)+32 \delta(-7+36 \nu)\right)(S \Sigma) \\
& +\left(16 \delta^{2} \kappa_{+}(-4+9 \nu)+24 \delta^{2}(-1+12 \nu)-16 \delta \kappa_{-}(-4+17 \nu)\right) \Sigma^{2} .
\end{aligned}
$$

\section{Angular momentum flux}

The coefficients for the radiated angular momentum in (42)-(45) are given by

$$
\begin{aligned}
& g_{3}^{0 i}=\boldsymbol{S}^{i}\left(-120(n v)^{4}+264(n v)^{2} \boldsymbol{v}^{2}-160\left(\boldsymbol{v}^{2}\right)^{2}\right)+\boldsymbol{v}^{i}\left((v S)\left(-348(n v)^{2}+160 \boldsymbol{v}^{2}\right)+(n S)\left(780(n v)^{3}-444(n v) \boldsymbol{v}^{2}\right)\right. \\
& \left.+(n \Sigma)\left(420 \delta(n v)^{3}-228 \delta(n v) v^{2}\right)+\left(-204 \delta(n v)^{2}+88 \delta v^{2}\right)(v \Sigma)\right) \\
& +\boldsymbol{n}^{i}\left((v S)\left(120(n v)^{3}+84(n v) \boldsymbol{v}^{2}\right)+(n S)\left(-780(n v)^{2} \boldsymbol{v}^{2}+444\left(\boldsymbol{v}^{2}\right)^{2}\right)\right. \\
& \left.+(n \Sigma)\left(-420 \delta(n v)^{2} \boldsymbol{v}^{2}+228 \delta\left(\boldsymbol{v}^{2}\right)^{2}\right)+\left(120 \delta(n v)^{3}+12 \delta(n v) \boldsymbol{v}^{2}\right)(v \Sigma)\right) \\
& +\left(-120 \delta(n v)^{4}+192 \delta(n v)^{2} \boldsymbol{v}^{2}-88 \delta\left(v^{2}\right)^{2}\right) \Sigma^{i} \\
& g_{3}^{1 i}=S^{i}\left(76(n v)^{2}-72 v^{2}\right)+v^{i}(-175(n S)(n v)-76 \delta(n v)(n \Sigma)+113(v S)+44 \delta(v \Sigma)) \\
& +\boldsymbol{n}^{i}\left(-109(n v)(v S)+(n S)\left(45(n v)^{2}+122 \boldsymbol{v}^{2}\right)+(n \Sigma)\left(27 \delta(n v)^{2}+47 \delta v^{2}\right)-46 \delta(n v)(v \Sigma)\right) \\
& +\left(26 \delta(n v)^{2}-22 \delta v^{2}\right) \Sigma^{i} \\
& g_{3}^{2 i}=\boldsymbol{n}^{i}(2(n S)-\delta(n \Sigma))-2 \boldsymbol{S}^{i}+\delta \boldsymbol{\Sigma}^{i}, \\
& g_{4}^{0 i}=(\boldsymbol{v} \times \boldsymbol{S})^{i}\left[\left(-24-12 \kappa_{+}\right)(n v)(v S)+(n S)\left(\left(120+60 \kappa_{+}\right)(n v)^{2}+\left(-72-36 \kappa_{+}\right) \boldsymbol{v}^{2}\right)\right. \\
& \left.+(n \Sigma)\left(\left(60 \delta-30 \kappa_{-}+30 \delta \kappa_{+}\right)(n v)^{2}+\left(-36 \delta+18 \kappa_{-}-18 \delta \kappa_{+}\right) v^{2}\right)+\left(-12 \delta+6 \kappa_{-}-6 \delta \kappa_{+}\right)(n v)(v \Sigma)\right] \\
& +(\boldsymbol{v} \times \boldsymbol{\Sigma})^{i}\left\{\left(-12 \delta+6 \kappa_{-}-6 \delta \kappa_{+}\right)(n v)(v S)+(n S)\left(\left(60 \delta-30 \kappa_{-}+30 \delta \kappa_{+}\right)(n v)^{2}\right.\right. \\
& \left.+\left(-36 \delta+18 \kappa_{-}-18 \delta \kappa_{+}\right) \boldsymbol{v}^{2}\right)+(n \Sigma)\left[\left(-30 \delta \kappa_{-}+\kappa_{+}(30-60 \nu)-120 \nu\right)(n v)^{2}\right. \\
& \left.\left.+\left(18 \delta \kappa_{-}+72 \nu+\kappa_{+}(-18+36 \nu)\right) v^{2}\right]+\left(6 \delta \kappa_{-}+24 \nu+\kappa_{+}(-6+12 \nu)\right)(n v)(v \Sigma)\right\} \\
& +(\boldsymbol{n} \times \boldsymbol{v})^{i}\left\{\left(-360-180 \kappa_{+}\right)(n S)(n v)(v S)+\left(-180 \delta+90 \kappa_{-}-90 \delta \kappa_{+}\right)(n v)(n \Sigma)(v S)\right. \\
& +\left(24+12 \kappa_{+}\right)(v S)^{2}+(n S)^{2}\left(\left(840+420 \kappa_{+}\right)(n v)^{2}+\left(-240-120 \kappa_{+}\right) v^{2}\right)+S^{2}\left(\left(-120-60 \kappa_{+}\right)(n v)^{2}\right. \\
& \left.+\left(48+24 \kappa_{+}\right) \boldsymbol{v}^{2}\right)+(n S)(n \Sigma)\left(\left(840 \delta-420 \kappa_{-}+420 \delta \kappa_{+}\right)(n v)^{2}+\left(-240 \delta+120 \kappa_{-}-120 \delta \kappa_{+}\right) \boldsymbol{v}^{2}\right) \\
& +(S \Sigma)\left(\left(-120 \delta+60 \kappa_{-}-60 \delta \kappa_{+}\right)(n v)^{2}+\left(48 \delta-24 \kappa_{-}+24 \delta \kappa_{+}\right) \boldsymbol{v}^{2}\right) \\
& +(n \Sigma)^{2}\left[\left(-210 \delta \kappa_{-}+\kappa_{+}(210-420 \nu)-840 \nu\right)(n v)^{2}\right. \\
& \left.+\left(60 \delta \kappa_{-}+240 \nu+60 \kappa_{+}(-1+2 \nu)\right) v^{2}\right]+\left(-180 \delta+90 \kappa_{-}-90 \delta \kappa_{+}\right)(n S)(n v)(v \Sigma) \\
& +\left(90 \delta \kappa_{-}+360 \nu+90 \kappa_{+}(-1+2 \nu)\right)(n v)(n \Sigma)(v \Sigma)+\left(24 \delta-12 \kappa_{-}+12 \delta \kappa_{+}\right)(v S)(v \Sigma) \\
& +\left(-6 \delta \kappa_{-}+\kappa_{+}(6-12 \nu)-24 \nu\right)(v \Sigma)^{2}+\left[\left(30 \delta \kappa_{-}+120 \nu+\kappa_{+}(-30+60 \nu)\right)(n v)^{2}\right. \\
& \left.\left.+\left(-12 \delta \kappa_{-}+\kappa_{+}(12-24 \nu)-48 \nu\right) \boldsymbol{v}^{2}\right] \boldsymbol{\Sigma}^{2}\right\}
\end{aligned}
$$




$$
\begin{aligned}
& g_{4}^{1 i}=\left(\left(-48-24 \kappa_{+}\right)(n S)+\left(-24 \delta+12 \kappa_{-}-12 \delta \kappa_{+}\right)(n \Sigma)\right)(\boldsymbol{v} \times \boldsymbol{S})^{i} \\
& +\left[\left(-24 \delta+12 \kappa_{-}-12 \delta \kappa_{+}\right)(n S)+\left(-2+12 \delta \kappa_{-}+48 \nu+\kappa_{+}(-12+24 \nu)\right)(n \Sigma)\right](\boldsymbol{v} \times \boldsymbol{\Sigma})^{i} \\
& +(\boldsymbol{n} \times \boldsymbol{S})^{i}\left(\left(-24-12 \kappa_{+}\right)(n S)(n v)+\left(-12 \delta+6 \kappa_{-}-6 \delta \kappa_{+}\right)(n v)(n \Sigma)\right. \\
& \left.+\left(24+12 \kappa_{+}\right)(v S)+\left(12 \delta-6 \kappa_{-}+6 \delta \kappa_{+}\right)(v \Sigma)\right) \\
& +(\boldsymbol{n} \times \Sigma)^{i}\left[\left(-12 \delta+6 \kappa_{-}-6 \delta \kappa_{+}\right)(n S)(n v)+\left(6 \delta \kappa_{-}+24 \nu+\kappa_{+}(-6+12 \nu)\right)(n v)(n \Sigma)\right. \\
& \left.+\left(12 \delta-6 \kappa_{-}+6 \delta \kappa_{+}\right)(v S)+\left(2-6 \delta \kappa_{-}+\kappa_{+}(6-12 \nu)-24 \nu\right)(v \Sigma)\right] \\
& +(\boldsymbol{n} \times \boldsymbol{v})^{i}\left[\left(-360-180 \kappa_{+}\right)(n S)^{2}+\left(-360 \delta+180 \kappa_{-}-180 \delta \kappa_{+}\right)(n S)(n \Sigma)\right. \\
& +\left(90 \delta \kappa_{-}+360 \nu+90 \kappa_{+}(-1+2 \nu)\right)(n \Sigma)^{2}+\left(96+48 \kappa_{+}\right) S^{2}+\left(96 \delta-48 \kappa_{-}+48 \delta \kappa_{+}\right)(S \Sigma) \\
& \left.+\left(2-24 \delta \kappa_{-}+\kappa_{+}(24-48 \nu)-96 \nu\right) \Sigma^{2}\right] \\
& g_{5}^{0 i}=\boldsymbol{S}^{i}\left(-105(1+40 \nu)(n v)^{6}+15(-43+716 \nu)(n v)^{4} \boldsymbol{v}^{2}+(2589-10608 \nu)(n v)^{2}\left(\boldsymbol{v}^{2}\right)^{2}+(-1607+4004 \nu)\left(\boldsymbol{v}^{2}\right)^{3}\right) \\
& +v^{i}\left[(v S)\left((2145-7740 \nu)(n v)^{4}+30(-65+334 \nu)(n v)^{2} v^{2}+(1607-4004 \nu)\left(v^{2}\right)^{2}\right)\right. \\
& +(n \Sigma)\left(210 \delta(-4+35 \nu)(n v)^{5}-30 \delta(-49+388 \nu)(n v)^{3} v^{2}+6 \delta(-217+787 \nu)(n v)\left(v^{2}\right)^{2}\right) \\
& +(n S)\left(1260(-1+6 \nu)(n v)^{5}-30(-53+496 \nu)(n v)^{3} v^{2}+(-2874+8376 \nu)(n v)\left(v^{2}\right)^{2}\right) \\
& \left.+\left(-15 \delta(-79+455 \nu)(n v)^{4}+6 \delta(-147+1427 \nu)(n v)^{2} v^{2}+\delta(563-2477 \nu)\left(v^{2}\right)^{2}\right)(v \Sigma)\right] \\
& +\boldsymbol{n}^{i}\left[(v S)\left(105(1+40 \nu)(n v)^{5}-1500(1+2 \nu)(n v)^{3} \boldsymbol{v}^{2}+(-639+588 \nu)(n v)\left(\boldsymbol{v}^{2}\right)^{2}\right)\right. \\
& +(n S)\left(-1260(-1+6 \nu)(n v)^{4} \boldsymbol{v}^{2}+30(-53+496 \nu)(n v)^{2}\left(\boldsymbol{v}^{2}\right)^{2}+(2874-8376 \nu)\left(\boldsymbol{v}^{2}\right)^{3}\right) \\
& +(n \Sigma)\left(-210 \delta(-4+35 \nu)(n v)^{4} \boldsymbol{v}^{2}+30 \delta(-49+388 \nu)(n v)^{2}\left(\boldsymbol{v}^{2}\right)^{2}-6 \delta(-217+787 \nu)\left(v^{2}\right)^{3}\right) \\
& \left.+\left(525 \delta(5+13 \nu)(n v)^{5}-270 \delta(20+29 \nu)(n v)^{3} v^{2}+3 \delta(559+571 \nu)(n v)\left(v^{2}\right)^{2}\right)(v \Sigma)\right] \\
& +\boldsymbol{\Sigma}^{i}\left(-525 \delta(5+13 \nu)(n v)^{6}+15 \delta(281+977 \nu)(n v)^{4} v^{2}\right. \\
& \left.-15 \delta(53+685 \nu)(n v)^{2}\left(v^{2}\right)^{2}+\delta(-563+2477 \nu)\left(v^{2}\right)^{3}\right), \\
& g_{5}^{1 i}=\boldsymbol{S}^{i}\left((-8577+7320 \nu)(n v)^{4}+(7022-15068 \nu)(n v)^{2} \boldsymbol{v}^{2}+7(-155+972 \nu)\left(\boldsymbol{v}^{2}\right)^{2}\right) \\
& +\boldsymbol{v}^{i}\left[(v S)\left((-588+10982 \nu)(n v)^{2}+(3138-8238 \nu) \boldsymbol{v}^{2}\right)\right. \\
& +(n \Sigma)\left(-9 \delta(1519+216 \nu)(n v)^{3}+\delta(4789+1994 \nu)(n v) v^{2}\right) \\
& +(n S)\left(-6(2750+1699 \nu)(n v)^{3}+(3542+6802 \nu)(n v) \boldsymbol{v}^{2}\right) \\
& \left.+\left(\delta(4313+1486 \nu)(n v)^{2}-17 \delta(39+112 \nu) v^{2}\right)(v \Sigma)\right] \\
& +\boldsymbol{n}^{i}\left[(v S)\left((12570-10914 \nu)(n v)^{3}+(-12836+9162 \nu)(n v) \boldsymbol{v}^{2}\right)\right. \\
& +(n S)\left(-3(1381+574 \nu)(n v)^{4}+6(3919+2760 \nu)(n v)^{2} v^{2}+(-6057-11494 \nu)\left(\boldsymbol{v}^{2}\right)^{2}\right) \\
& +(n \Sigma)\left(3 \delta(-807+\nu)(n v)^{4}+3 \delta(5909+1242 \nu)(n v)^{2} v^{2}-\delta(6364+3679 \nu)\left(\boldsymbol{v}^{2}\right)^{2}\right) \\
& \left.+\left(-3 \delta(-759+296 \nu)(n v)^{3}+3 \delta(-1729+674 \nu)(n v) v^{2}\right)(v \Sigma)\right] \\
& +\boldsymbol{\Sigma}^{i}\left(-3 \delta(-179+361 \nu)(n v)^{4}-10 \delta(336+85 \nu)(n v)^{2} \boldsymbol{v}^{2}+\delta(2023+1117 \nu)\left(\boldsymbol{v}^{2}\right)^{2}\right) \\
& g_{5}^{2 i}=\boldsymbol{S}^{i}\left(-2(7711+272 \nu)(n v)^{2}+(16218+2296 \nu) \mathbf{v}^{2}\right) \\
& +\boldsymbol{v}^{i}(8(6227+386 \nu)(n S)(n v)+36 \delta(685+36 \nu)(n v)(n \Sigma)-2(14701+728 \nu)(v S)-10 \delta(1501+54 \nu)(v \Sigma)) \\
& +\boldsymbol{n}^{i}\left[(27954+536 \nu)(n v)(v S)+(n \Sigma)\left(-2 \delta(5157+847 \nu)(n v)^{2}-2 \delta(7151+163 \nu) \boldsymbol{v}^{2}\right)\right. \\
& \left.+(n S)\left(-4(5115+413 \nu)(n v)^{2}-12(2392+189 \nu) v^{2}\right)-2 \delta(-7561+96 \nu)(n v)(v \Sigma)\right] \\
& +\boldsymbol{\Sigma}^{i}\left(2 \delta(-4040+541 \nu)(n v)^{2}+2 \delta(3962+187 \nu) \boldsymbol{v}^{2}\right), \\
& g_{5}^{3 i}=\boldsymbol{n}^{i}(-8(265+31 \nu)(n S)-20 \delta(-12+7 \nu)(n \Sigma))+8(265+31 \nu) \boldsymbol{S}^{i}+20 \delta(-12+7 \nu) \boldsymbol{\Sigma}^{i},
\end{aligned}
$$




$$
\begin{aligned}
& g_{6}^{0 i}=(\boldsymbol{v} \times \boldsymbol{S})^{i}\left\{( v S ) \left[\left(-3020 \delta \kappa_{-}-280 \kappa_{+}(1+\nu)-80(-52+7 \nu)\right)(n v)^{3}\right.\right. \\
& \left.+\left(1916 \delta \kappa_{-}+\kappa_{+}(680-252 \nu)-56(68+9 \nu)\right)(n v) v^{2}\right] \\
& +(n \Sigma)\left[\left(140 \delta \kappa_{+}(-23+2 \nu)+280 \delta(-1+2 \nu)-140 \kappa_{-}(-23+88 \nu)\right)(n v)^{4}\right. \\
& +\left(-10 \delta \kappa_{+}(-258+19 \nu)-20 \delta(-169+19 \nu)+30 \kappa_{-}(-86+349 \nu)\right)(n v)^{2} v^{2} \\
& \left.+\left(\kappa_{-}(504-954 \nu)+14 \delta \kappa_{+}(-36+19 \nu)+4 \delta(-337+133 \nu)\right)\left(v^{2}\right)^{2}\right] \\
& +(n S)\left[\left(6020 \delta \kappa_{-}+1120(-11+\nu)+140 \kappa_{+}(-3+4 \nu)\right)(n v)^{4}+\left(16280-5140 \delta \kappa_{-}\right.\right. \\
& \left.\left.+\kappa_{+}(20-380 \nu)-760 \nu\right)(n v)^{2} v^{2}+\left(344 \delta \kappa_{-}+8(-449+133 \nu)+\kappa_{+}(-664+532 \nu)\right)\left(v^{2}\right)^{2}\right] \\
& +\left[\left(-10 \delta \kappa_{+}(-137+14 \nu)-20 \delta(57+14 \nu)+\kappa_{-}(-1370+6180 \nu)\right)(n v)^{3}\right. \\
& \left.\left.+\left(\kappa_{-}(618-3706 \nu)-252 \delta \nu-6 \delta \kappa_{+}(103+21 \nu)\right)(n v) v^{2}\right](v \Sigma)\right\} \\
& +(\boldsymbol{v} \times \boldsymbol{\Sigma})^{i}\left\{( v S ) \left[\left(-20 \delta(-223+14 \nu)-10 \delta \kappa_{+}(-137+14 \nu)\right.\right.\right. \\
& \left.\left.+\kappa_{-}(-1370+6180 \nu)\right)(n v)^{3}+\left(\kappa_{-}(618-3706 \nu)-84 \delta(46+3 \nu)-6 \delta \kappa_{+}(103+21 \nu)\right)(n v) v^{2}\right] \\
& +(n S)\left[\left(560 \delta(-25+\nu)+140 \delta \kappa_{+}(-23+2 \nu)-140 \kappa_{-}(-23+88 \nu)\right)(n v)^{4}\right. \\
& +\left(-20 \delta(-813+19 \nu)-10 \delta \kappa_{+}(-258+19 \nu)+30 \kappa_{-}(-86+349 \nu)\right)(n v)^{2} v^{2} \\
& \left.+\left(\kappa_{-}(504-954 \nu)+14 \delta \kappa_{+}(-36+19 \nu)+4 \delta(-687+133 \nu)\right)\left(v^{2}\right)^{2}\right] \\
& +(n \Sigma)\left[\left(-140 \delta \kappa_{-}(-23+45 \nu)-140 \kappa_{+}\left(23-91 \nu+4 \nu^{2}\right)-280\left(7-58 \nu+4 \nu^{2}\right)\right)(n v)^{4}\right. \\
& +\left(10 \delta \kappa_{-}(-258+533 \nu)+40\left(84-568 \nu+19 \nu^{2}\right)+10 \kappa_{+}\left(258-1049 \nu+38 \nu^{2}\right)\right)(n v)^{2} v^{2} \\
& \left.+\left(\delta \kappa_{-}(504-610 \nu)+\kappa_{+}\left(-504+1618 \nu-532 \nu^{2}\right)-8\left(63-568 \nu+133 \nu^{2}\right)\right)\left(v^{2}\right)^{2}\right] \\
& +\left[\left(10 \delta \kappa_{-}(-137+316 \nu)+40\left(-21-83 \nu+14 \nu^{2}\right)+10 \kappa_{+}\left(137-590 \nu+28 \nu^{2}\right)\right)(n v)^{3}\right. \\
& \left.\left.+\left(-2 \delta \kappa_{-}(-309+895 \nu)+56\left(-1+77 \nu+9 \nu^{2}\right)+\kappa_{+}\left(-618+3026 \nu+252 \nu^{2}\right)\right)(n v) \boldsymbol{v}^{2}\right](v \Sigma)\right\} \\
& +(\boldsymbol{n} \times \boldsymbol{v})^{i}\left\{( v S ) ^ { 2 } \left[\left(2280 \delta \kappa_{-}+40(-3+142 \nu)+\kappa_{+}(-2100+2840 \nu)\right)(n v)^{2}\right.\right. \\
& \left.+\left(-512 \delta \kappa_{-}+8(-99+19 \nu)+\kappa_{+}(-100+76 \nu)\right) v^{2}\right] \\
& +(n \Sigma)(v S)\left[\left(-70 \delta \kappa_{+}(-113+128 \nu)-140 \delta(1+128 \nu)\right.\right. \\
& \left.+70 \kappa_{-}(-113+412 \nu)\right)(n v)^{3}+\left(\kappa_{-}(1730-10090 \nu)+10 \delta \kappa_{+}(-173+157 \nu)\right. \\
& \left.+20 \delta(36+157 \nu))(n v) \boldsymbol{v}^{2}\right]+(n S)(v S)\left[\left(7560-9940 \delta \kappa_{-}-35840 \nu-280 \kappa_{+}(-21+64 \nu)\right)(n v)^{3}\right. \\
& \left.+\left(40+4260 \delta \kappa_{-}+6280 \nu+\kappa_{+}(800+3140 \nu)\right)(n v) v^{2}\right] \\
& +S^{2}\left[\left(4060+1400 \delta \kappa_{-}-8400 \nu-70 \kappa_{+}(-23+60 \nu)\right)(n v)^{4}\right. \\
& +\left(-1420 \delta \kappa_{-}+40(-90+181 \nu)+20 \kappa_{+}(-69+181 \nu)\right)(n v)^{2} v^{2} \\
& \left.+\left(1068+248 \delta \kappa_{-}+\kappa_{+}(486-616 \nu)-1232 \nu\right)\left(v^{2}\right)^{2}\right] \\
& +(n S)^{2}\left[\left(8820 \delta \kappa_{-}+1260(-13+40 \nu)+630 \kappa_{+}(-13+40 \nu)\right)(n v)^{4}+\left(-4900 \delta \kappa_{-}\right.\right. \\
& \left.\left.-420 \kappa_{+}(-7+29 \nu)-280(-49+87 \nu)\right)(n v)^{2} \boldsymbol{v}^{2}+\left(-4660-140 \delta \kappa_{-}+3600 \nu+30 \kappa_{+}(-59+60 \nu)\right)\left(\boldsymbol{v}^{2}\right)^{2}\right] \\
& +(n S)(n \Sigma)\left[\left(-1890 \kappa_{-}(-9+32 \nu)+630 \delta \kappa_{+}(-27+40 \nu)+1260 \delta(-13+40 \nu)\right)(n v)^{4}\right. \\
& +\left(-840 \delta(-21+29 \nu)-140 \delta \kappa_{+}(-56+87 \nu)+140 \kappa_{-}(-56+227 \nu)\right)(n v)^{2} v^{2} \\
& \left.+\left(\kappa_{-}(1630-1240 \nu)+180 \delta(-29+20 \nu)+10 \delta \kappa_{+}(-163+180 \nu)\right)\left(v^{2}\right)^{2}\right] \\
& +(S \Sigma)\left[\left(-420 \delta(-19+20 \nu)-210 \delta \kappa_{+}(-1+20 \nu)-70 \kappa_{-}(3+20 \nu)\right)(n v)^{4}\right. \\
& +\left(40 \delta(-174+181 \nu)+20 \delta \kappa_{+}(2+181 \nu)+\kappa_{-}(-40+2060 \nu)\right)(n v)^{2} v^{2} \\
& \left.+\left(\kappa_{-}(-238-376 \nu)-14 \delta \kappa_{+}(-17+44 \nu)-4 \delta(-351+308 \nu)\right)\left(v^{2}\right)^{2}\right]
\end{aligned}
$$




$$
\begin{aligned}
& +(n \Sigma)^{2}\left[\left(-1260 \nu(-13+40 \nu)-315 \delta \kappa_{-}(-27+68 \nu)-315 \kappa_{+}\left(27-122 \nu+80 \nu^{2}\right)\right)(n v)^{4}\right. \\
& +\left(70 \delta \kappa_{-}(-56+157 \nu)+280\left(14-77 \nu+87 \nu^{2}\right)+70 \kappa_{+}\left(56-269 \nu+174 \nu^{2}\right)\right)(n v)^{2} \boldsymbol{v}^{2} \\
& \left.+\left(\delta \kappa_{-}(815-760 \nu)-20\left(28-289 \nu+180 \nu^{2}\right)-5 \kappa_{+}\left(163-478 \nu+360 \nu^{2}\right)\right)\left(v^{2}\right)^{2}\right] \\
& +(v S)\left[\left(\kappa_{-}(4380-11960 \nu)+80 \delta(23+71 \nu)+20 \delta \kappa_{+}(-219+142 \nu)\right)(n v)^{2}\right. \\
& \left.+\left(8 \delta(-134+19 \nu)+4 \delta \kappa_{+}(103+19 \nu)+\kappa_{-}(-412+1972 \nu)\right) v^{2}\right](v \Sigma) \\
& +(n S)\left[\left(-70 \delta \kappa_{+}(-113+128 \nu)-140 \delta(-13+128 \nu)+70 \kappa_{-}(-113+412 \nu)\right)(n v)^{3}\right. \\
& \left.+\left(\kappa_{-}(1730-10090 \nu)+10 \delta \kappa_{+}(-173+157 \nu)+20 \delta(8+157 \nu)\right)(n v) v^{2}\right](v \Sigma) \\
& +(n \Sigma)\left[\left(70 \delta \kappa_{-}(-113+270 \nu)+280\left(-21+15 \nu+128 \nu^{2}\right)+70 \kappa_{+}\left(113-496 \nu+256 \nu^{2}\right)\right)(n v)^{3}\right. \\
& \left.+\left(-10 \delta \kappa_{-}(-173+583 \nu)-40\left(-21+43 \nu+157 \nu^{2}\right)-10 \kappa_{+}\left(173-929 \nu+314 \nu^{2}\right)\right)(n v) \boldsymbol{v}^{2}\right](v \Sigma) \\
& +\left[\left(-10 \delta \kappa_{-}(-219+370 \nu)-40\left(-49+88 \nu+142 \nu^{2}\right)-10 \kappa_{+}\left(219-808 \nu+284 \nu^{2}\right)\right)(n v)^{2}\right. \\
& \left.+\left(2 \delta \kappa_{-}(-103+237 \nu)+\kappa_{+}\left(206-886 \nu-76 \nu^{2}\right)-8\left(35-162 \nu+19 \nu^{2}\right)\right) v^{2}\right](v \Sigma)^{2} \\
& +\left[\left(35 \delta \kappa_{-}(-3+20 \nu)+140\left(28-85 \nu+60 \nu^{2}\right)+35 \kappa_{+}\left(3-26 \nu+120 \nu^{2}\right)\right)(n v)^{4}\right. \\
& +\left(-10 \delta \kappa_{-}(2+39 \nu)+\kappa_{+}\left(20+350 \nu-3620 \nu^{2}\right)-40\left(84-251 \nu+181 \nu^{2}\right)\right)(n v)^{2} \boldsymbol{v}^{2} \\
& \left.\left.+\left(\delta \kappa_{-}(-119+60 \nu)+4\left(84-421 \nu+308 \nu^{2}\right)+\kappa_{+}\left(119-298 \nu+616 \nu^{2}\right)\right)\left(\boldsymbol{v}^{2}\right)^{2}\right] \boldsymbol{\Sigma}^{2}\right\}, \\
& g_{6}^{1 i}=(\boldsymbol{S} \times \boldsymbol{\Sigma})^{i}\left(-792 \delta(n v)^{3}+904 \delta(n v) \boldsymbol{v}^{2}\right)+(\boldsymbol{v} \times \boldsymbol{S})^{i}\left\{\left(6648-1492 \delta \kappa_{-}+48 \nu+\kappa_{+}(-92+24 \nu)\right)(n v)(v S)\right. \\
& +(n S)\left[\left(5772 \delta \kappa_{-}-144 \kappa_{+}(54+11 \nu)-36(647+88 \nu)\right)(n v)^{2}\right. \\
& \left.+\left(-2824 \delta \kappa_{-}+4(2530+279 \nu)+\kappa_{+}(3838+558 \nu)\right) v^{2}\right] \\
& +(n \Sigma)\left[\left(\kappa_{-}(6774-10752 \nu)-12 \delta(877+132 \nu)-6 \delta \kappa_{+}(1129+132 \nu)\right)(n v)^{2}\right. \\
& \left.+\left(\delta \kappa_{+}(3331+279 \nu)+\delta(3779+558 \nu)+\kappa_{-}(-3331+5369 \nu)\right) v^{2}\right] \\
& \left.+\left(4 \delta \kappa_{+}(175+3 \nu)+8 \delta(536+3 \nu)+\kappa_{-}(-700+2972 \nu)\right)(n v)(v \Sigma)\right\} \\
& +(\boldsymbol{v} \times \boldsymbol{\Sigma})^{i}\left\{\left(4 \delta \kappa_{+}(175+3 \nu)+2 \delta(1963+12 \nu)+\kappa_{-}(-700+2972 \nu)\right)(n v)(v S)\right. \\
& +(n S)\left[\left(\kappa_{-}(6774-10752 \nu)-72 \delta(235+22 \nu)-6 \delta \kappa_{+}(1129+132 \nu)\right)(n v)^{2}\right. \\
& \left.+\left(\delta \kappa_{+}(3331+279 \nu)+\delta(9419+558 \nu)+\kappa_{-}(-3331+5369 \nu)\right) v^{2}\right] \\
& +(n \Sigma)\left[\left(-6 \delta \kappa_{-}(-1129+830 \nu)+6 \kappa_{+}\left(-1129+3088 \nu+264 \nu^{2}\right)+6(-447+5230 \nu\right.\right. \\
& \left.\left.+528 \nu^{2}\right)\right)(n v)^{2}+\left(\delta \kappa_{-}(-3331+2545 \nu)+\kappa_{+}\left(3331-9207 \nu-558 \nu^{2}\right)-4(-381+3974 \nu\right. \\
& \left.\left.\left.+279 \nu^{2}\right)\right) \boldsymbol{v}^{2}\right]+\left(20 \delta \kappa_{-}(-35+74 \nu)-4 \kappa_{+}\left(-175+720 \nu+6 \nu^{2}\right)\right. \\
& \left.\left.-6\left(-205+1695 \nu+8 \nu^{2}\right)\right)(n v)(v \Sigma)\right\}+(\boldsymbol{n} \times \boldsymbol{S})^{i}\left\{( v S ) \left[\left(-8016+2238 \delta \kappa_{-}\right.\right.\right. \\
& \left.\left.+1884 \nu+\kappa_{+}(-966+942 \nu)\right)(n v)^{2}+\left(8012-862 \delta \kappa_{-}+168 \nu+\kappa_{+}(448+84 \nu)\right) v^{2}\right] \\
& +(n \Sigma)\left[\left(6 \delta(399+103 \nu)+3 \delta \kappa_{+}(1135+103 \nu)+\kappa_{-}(-3405+8943 \nu)\right)(n v)^{3}+\left(\kappa_{-}(2295-5601 \nu)\right.\right. \\
& \left.\left.-15 \delta \kappa_{+}(153+65 \nu)-6 \delta(782+325 \nu)\right)(n v) v^{2}\right]+(n S)\left[\left(13632-4626 \delta \kappa_{-}\right.\right. \\
& \left.\left.+1236 \nu+\kappa_{+}(2184+618 \nu)\right)(n v)^{3}+\left(3288 \delta \kappa_{-}-6 \kappa_{+}(217+325 \nu)-12(1371+325 \nu)\right)(n v) \boldsymbol{v}^{2}\right] \\
& +\left[\left(\kappa_{-}(1602-4947 \nu)+3 \delta \kappa_{+}(-534+157 \nu)+6 \delta(-448+157 \nu)\right)(n v)^{2}+(3 \delta(1133+28 \nu)\right. \\
& \left.\left.\left.+\delta \kappa_{+}(655+42 \nu)+\kappa_{-}(-655+1682 \nu)\right) v^{2}\right](v \Sigma)\right\} \\
& +(\boldsymbol{n} \times \boldsymbol{\Sigma})^{i}\left\{( v S ) \left[\left(\kappa_{-}(1602-4947 \nu)+6 \delta(-940+157 \nu)+3 \delta \kappa_{+}(-534+157 \nu)\right)(n v)^{2}\right.\right. \\
& \left.+\left(\delta \kappa_{+}(655+42 \nu)+\delta(4769+84 \nu)+\kappa_{-}(-655+1682 \nu)\right) v^{2}\right]+(n S)\left[\left(3 \delta \kappa_{+}(1135+103 \nu)\right.\right. \\
& \left.+6 \delta(2493+103 \nu)+\kappa_{-}(-3405+8943 \nu)\right)(n v)^{3}+\left(\kappa_{-}(2295-5601 \nu)-15 \delta \kappa_{+}(153+65 \nu)\right.
\end{aligned}
$$




$$
\begin{aligned}
& \left.-6 \delta(2468+325 \nu))(n v) \boldsymbol{v}^{2}\right]+(n \Sigma)\left[\left(3 \delta \kappa_{-}(-1135+1439 \nu)+\kappa_{+}\left(3405-11127 \nu-618 \nu^{2}\right)\right.\right. \\
& \left.-12\left(-380+1779 \nu+103 \nu^{2}\right)\right)(n v)^{3}+\left(-9 \delta \kappa_{-}(-255+257 \nu)+12\left(-394+1839 \nu+325 \nu^{2}\right)\right. \\
& \left.\left.+3 \kappa_{+}\left(-765+2301 \nu+650 \nu^{2}\right)\right)(n v) \boldsymbol{v}^{2}\right]+\left[\left(-9 \delta \kappa_{-}(-178+301 \nu)+\kappa_{+}\left(-1602+5913 \nu-942 \nu^{2}\right)\right.\right. \\
& \left.-6\left(269-1702 \nu+314 \nu^{2}\right)\right)(n v)^{2}+\left(5 \delta \kappa_{-}(-131+164 \nu)+\kappa_{+}\left(655-2130 \nu-84 \nu^{2}\right)\right. \\
& \left.\left.\left.-2\left(-855+4373 \nu+84 \nu^{2}\right)\right) \boldsymbol{v}^{2}\right](v \Sigma)\right\}+(\boldsymbol{n} \times \boldsymbol{v})^{i}\left\{\left(-11784 \delta \kappa_{-}+36 \kappa_{+}(767+243 \nu)\right.\right. \\
& +24(3610+729 \nu))(n S)(n v)(v S)+\left(42 \kappa_{-}(-469+457 \nu)+12 \delta(3049+729 \nu)\right. \\
& \left.+6 \delta \kappa_{+}(3283+729 \nu)\right)(n v)(n \Sigma)(v S)+\left(1290 \delta \kappa_{-}-6 \kappa_{+}(598+333 \nu)\right. \\
& -4(2587+999 \nu))(v S)^{2}+(S \Sigma)\left[\left(60 \delta \kappa_{+}(135+53 \nu)\right.\right. \\
& \left.+24 \delta(574+265 \nu)+\kappa_{-}(-8100+5316 \nu)\right)(n v)^{2}+\left(\kappa_{-}(1476-2656 \nu)-36 \delta \kappa_{+}(41+50 \nu)\right. \\
& \left.-50 \delta(115+72 \nu)) \boldsymbol{v}^{2}\right]+S^{2}\left[\left(-2124 \delta \kappa_{-}+24(543+265 \nu)+\kappa_{+}(5976+3180 \nu)\right)(n v)^{2}\right. \\
& \left.+\left(1114 \delta \kappa_{-}-2 \kappa_{+}(181+900 \nu)-4(521+900 \nu)\right) v^{2}\right]+(n S)(n \Sigma)\left[\left(\kappa_{-}(78072-78042 \nu)\right.\right. \\
& \left.-6 \delta \kappa_{+}(13012+2797 \nu)-12 \delta(13956+2797 \nu)\right)(n v)^{2}+\left(6 \delta \kappa_{+}(2653+1102 \nu)+12 \delta(4023+1102 \nu)\right. \\
& \left.\left.+6 \kappa_{-}(-2653+3714 \nu)\right) v^{2}\right]+(n S)^{2}\left[\left(23706 \delta \kappa_{-}-6 \kappa_{+}(9061+2797 \nu)-6(29683+5594 \nu)\right)(n v)^{2}\right. \\
& \left.+\left(-7224 \delta \kappa_{-}+6(7003+2204 \nu)+\kappa_{+}(8694+6612 \nu)\right) \boldsymbol{v}^{2}\right]+(n \Sigma)^{2}\left[\left(-3 \delta \kappa_{-}(-13012+5105 \nu)\right.\right. \\
& \left.+6\left(-1869+26316 \nu+5594 \nu^{2}\right)+\kappa_{+}\left(-39036+93387 \nu+16782 \nu^{2}\right)\right)(n v)^{2}+\left(3 \delta \kappa_{-}(-2653+1306 \nu)\right. \\
& \left.\left.+\kappa_{+}\left(7959-19836 \nu-6612 \nu^{2}\right)-6\left(-1519+9138 \nu+2204 \nu^{2}\right)\right) \nu^{2}\right] \\
& +\left(36 \delta(923+243 \nu)+42 \kappa_{-}(-469+457 \nu)+6 \delta \kappa_{+}(3283+729 \nu)\right)(n S)(n v)(v \Sigma) \\
& +\left(6 \delta \kappa_{-}(-3283+1235 \nu)-24\left(29+2235 \nu+729 \nu^{2}\right)-6 \kappa_{+}\left(-3283+7801 \nu+1458 \nu^{2}\right)\right)(n v)(n \Sigma)(v \Sigma) \\
& +\left(\kappa_{-}(4878-3162 \nu)-18 \delta \kappa_{+}(271+111 \nu)-2 \delta(2533+1998 \nu)\right)(v S)(v \Sigma) \\
& +\left(1922-38 \nu+3996 \nu^{2}-3 \delta \kappa_{-}(-813+97 \nu)+3 \kappa_{+}\left(-813+1723 \nu+666 \nu^{2}\right)\right)(v \Sigma)^{2} \\
& +\left[\left(786-15180 \nu-6360 \nu^{2}+6 \delta \kappa_{-}(-675+89 \nu)-6 \kappa_{+}\left(-675+1439 \nu+530 \nu^{2}\right)\right)(n v)^{2}\right. \\
& \left.\left.+\left(\delta \kappa_{-}(738-214 \nu)+24\left(-95+401 \nu+150 \nu^{2}\right)+2 \kappa_{+}\left(-369+845 \nu+900 \nu^{2}\right)\right) \boldsymbol{\nu}^{2}\right] \boldsymbol{\Sigma}^{2}\right\}, \\
& g_{6}^{2 i}=(\boldsymbol{v} \times \boldsymbol{S})^{i}\left[\left(-1268 \delta \kappa_{-}+12(941+30 \nu)+\kappa_{+}(6122+180 \nu)\right)(n S)\right. \\
& \left.+\left(5 \delta \kappa_{+}(739+18 \nu)+2 \delta(2543+90 \nu)+\kappa_{-}(-3695+2446 \nu)\right)(n \Sigma)\right] \\
& +(\boldsymbol{v} \times \boldsymbol{\Sigma})^{i}\left[\left(60 \delta(104+3 \nu)+5 \delta \kappa_{+}(739+18 \nu)+\kappa_{-}(-3695+2446 \nu)\right)(n S)\right. \\
& \left.+\left(\delta \kappa_{-}(-3695+1178 \nu)+\kappa_{+}\left(3695-8568 \nu-180 \nu^{2}\right)-4\left(-54+2831 \nu+90 \nu^{2}\right)\right)(n \Sigma)\right] \\
& +(\boldsymbol{n} \times \boldsymbol{S})^{i}\left[\left(-2522 \delta \kappa_{-}+8 \kappa_{+}(703+90 \nu)+8(1303+180 \nu)\right)(n S)(n v)+(18 \delta(159+40 \nu)\right. \\
& \left.+\delta \kappa_{+}(4073+360 \nu)+\kappa_{-}(-4073+4684 \nu)\right)(n v)(n \Sigma) \\
& +\left(1318 \delta \kappa_{-}+8(-926+27 \nu)+4 \kappa_{+}(-901+27 \nu)\right)(v S) \\
& \left.+\left(\kappa_{-}(2461-2690 \nu)+\delta \kappa_{+}(-2461+54 \nu)+2 \delta(-1111+54 \nu)\right)(v \Sigma)\right] \\
& +(\boldsymbol{n} \times \boldsymbol{\Sigma})^{i}\left[\left(24 \delta(187+30 \nu)+\delta \kappa_{+}(4073+360 \nu)+\kappa_{-}(-4073+4684 \nu)\right)(n S)(n v)\right. \\
& +\left(\delta \kappa_{-}(-4073+2162 \nu)+\kappa_{+}\left(4073-10308 \nu-720 \nu^{2}\right)-6\left(195+779 \nu+240 \nu^{2}\right)\right)(n v)(n \Sigma) \\
& +\left(\kappa_{-}(2461-2690 \nu)+\delta \kappa_{+}(-2461+54 \nu)+2 \delta(-1661+54 \nu)\right)(v S) \\
& \left.+\left(954+\delta \kappa_{-}(2461-1372 \nu)+4322 \nu-216 \nu^{2}+\kappa_{+}\left(-2461+6294 \nu-108 \nu^{2}\right)\right)(v \Sigma)\right] \\
& +(\boldsymbol{n} \times \boldsymbol{v})^{i}\left[\left(98500-6486 \delta \kappa_{-}+72 \nu+6 \kappa_{+}(7469+6 \nu)\right)(n S)^{2}\right. \\
& +\left(36 \delta \kappa_{+}(1425+\nu)+2 \delta(48905+36 \nu)+\kappa_{-}(-51300+25908 \nu)\right)(n S)(n \Sigma)
\end{aligned}
$$




$$
\begin{aligned}
& +\left(2558-96542 \nu-72 \nu^{2}+6 \delta \kappa_{-}(-4275+1078 \nu)-6 \kappa_{+}\left(-4275+9628 \nu+6 \nu^{2}\right)\right)(n \Sigma)^{2} \\
& +\left(1300 \delta \kappa_{-}+4 \kappa_{+}(-2924+3 \nu)+8(-2891+3 \nu)\right) S^{2}+\left(\kappa_{-}(12996-5212 \nu)\right. \\
& \left.+12 \delta \kappa_{+}(-1083+\nu)+8 \delta(-3124+3 \nu)\right)(S \Sigma)+\left(-2 \delta \kappa_{-}(-3249+653 \nu)\right. \\
& \left.\left.-6\left(201-4355 \nu+4 \nu^{2}\right)-2 \kappa_{+}\left(3249-7151 \nu+6 \nu^{2}\right)\right) \Sigma^{2}\right] .
\end{aligned}
$$

\section{Linear momentum and center-of-mass fluxes}

The coefficients for the expressions in Sec. IV C are given by

$$
\begin{aligned}
& h_{5}^{0 i}=(\boldsymbol{v} \times \boldsymbol{S})^{i}\left(-669 \delta(n v)^{3}+251 \delta(n v) \boldsymbol{v}^{2}\right)+(\boldsymbol{n} \times \boldsymbol{S})^{i}\left(2940 \delta(n v)^{4}-5307 \delta(n v)^{2} \boldsymbol{v}^{2}+2659 \delta\left(\boldsymbol{v}^{2}\right)^{2}\right) \\
& +(\boldsymbol{v} \times \boldsymbol{\Sigma})^{i}\left(-5331(n v)^{3}+2513(n v) \boldsymbol{v}^{2}+\nu\left(5952(n v)^{3}-3464(n v) \boldsymbol{v}^{2}\right)\right) \\
& +(\boldsymbol{n} \times \boldsymbol{\Sigma})^{i}\left(9195(n v)^{4}-9987(n v)^{2} \boldsymbol{v}^{2}+2260\left(\boldsymbol{v}^{2}\right)^{2}+\nu\left(-5442(n v)^{4}+8610(n v)^{2} \boldsymbol{v}^{2}-4594\left(\boldsymbol{v}^{2}\right)^{2}\right)\right) \\
& +(\boldsymbol{n} \times \boldsymbol{v})^{i}\left(-12(n v)^{3}(1541 \delta(n S)+431(n \Sigma))+3(n v)(5287 \delta(n S)+1087(n \Sigma)) \boldsymbol{v}^{2}\right. \\
& -2 v^{2}(2252 \delta(v S)+1481(v \Sigma))+(n v)^{2}(6348 \delta(v S)+6024(v \Sigma)) \\
& \left.+\nu\left(32658(n v)^{3}(n \Sigma)-27615(n v)(n \Sigma) v^{2}-15717(n v)^{2}(v \Sigma)+9247 v^{2}(v \Sigma)\right)\right) \\
& +\boldsymbol{v}^{i}\left(-21(n v)^{2}(463 \delta(S n v)+274(\Sigma n v))+\boldsymbol{v}^{2}(9049 \delta(\operatorname{Snv})\right. \\
& \left.+4780(\Sigma n v))+\nu\left(22089(n v)^{2}(\Sigma n v)-18265 v^{2}(\Sigma n v)\right)\right) \\
& +\boldsymbol{n}^{i}\left(-1056(n v) \boldsymbol{v}^{2}(13 \delta(S n v)+7(\Sigma n v))+(n v)^{3}(14604 \delta(S n v)\right. \\
& \left.+8304(\Sigma n v))+\nu\left(-31146(n v)^{3}(\Sigma n v)+27534(n v) \boldsymbol{v}^{2}(\Sigma n v)\right)\right) \text {, } \\
& h_{5}^{1 i}=496 \delta(n v)(\boldsymbol{v} \times \boldsymbol{S})^{i}+(-6422(n v)-40 \nu(n v))(\boldsymbol{v} \times \boldsymbol{\Sigma})^{i} \\
& +(\boldsymbol{n} \times \boldsymbol{\Sigma})^{i}\left(3557(n v)^{2}+\nu\left(4618(n v)^{2}-3372 \boldsymbol{v}^{2}\right)+1389 \boldsymbol{v}^{2}\right)+(\boldsymbol{n} \times \boldsymbol{S})^{i}\left(-2092 \delta(n v)^{2}+1056 \delta \boldsymbol{v}^{2}\right) \\
& +(\boldsymbol{n} \times \boldsymbol{v})^{i}((n v)(-1886 \delta(n S)+4930(n \Sigma))+\nu(2807(n v)(n \Sigma)-759(v \Sigma)) \\
& -120(\delta(v S)-5(v \Sigma)))+v^{i}(2568 \delta(S n v)+669(\Sigma n v)-2391 \nu(\Sigma n v)) \\
& +\boldsymbol{n}^{i}((n v)(-3460 \delta(S n v)-1537(\Sigma n v))+5563 \nu(n v)(\Sigma n v)) \text {, } \\
& h_{5}^{2 i}=-72 \delta(\boldsymbol{n} \times \boldsymbol{S})^{i}, \\
& h_{6}^{0 i}=\boldsymbol{S}^{i}\left\{(n v)^{3}\left[\left(1920 \delta+4032 \kappa_{-}+960 \delta \kappa_{+}\right)(n S)+\left(1536 \delta \kappa_{-}-384 \kappa_{+}(4+5 \nu)-60(-45+64 \nu)\right)(n \Sigma)\right]\right. \\
& +(v S)\left(228 \delta v^{2}+1104 \kappa_{-} v^{2}+114 \delta \kappa_{+} v^{2}\right)+(n v)\left[(n S)\left(-1776 \delta v^{2}-3192 \kappa_{-} v^{2}-888 \delta \kappa_{+} v^{2}\right)\right. \\
& \left.+(n \Sigma)\left(-1152 \delta \kappa_{-} v^{2}+48 \kappa_{+}(24+37 \nu) v^{2}+12(-103+296 \nu) v^{2}\right)\right] \\
& +\left(495 \delta \kappa_{-} v^{2}-6(-329+76 \nu) \boldsymbol{v}^{2}-3 \kappa_{+}(165+76 \nu) v^{2}\right)(v \Sigma)+(n v)^{2}\left[\left(-312 \delta-1962 \kappa_{-}-156 \delta \kappa_{+}\right)(v S)\right. \\
& \left.\left.+\left(-903 \delta \kappa_{-}+6(-549+104 \nu)+3 \kappa_{+}(301+104 \nu)\right)(v \Sigma)\right]\right\} \\
& +\left\{( n v ) ^ { 3 } \left[\left(1536 \delta \kappa_{-}-384 \kappa_{+}(4+5 \nu)-12(251+320 \nu)\right)(n S)\right.\right. \\
& \left.+\left(-192 \delta \kappa_{+}(8+5 \nu)-192 \kappa_{-}(-8+11 \nu)-60 \delta(19+32 \nu)\right)(n \Sigma)\right] \\
& +(v S)\left(495 \delta \kappa_{-} v^{2}-8(70+57 \nu) \boldsymbol{v}^{2}-3 \kappa_{+}(165+76 \nu) \boldsymbol{v}^{2}\right) \\
& +(n v)\left[(n \Sigma)\left(24 \delta \kappa_{+}(48+37 \nu) \boldsymbol{v}^{2}+24 \kappa_{-}(-48+59 \nu) \boldsymbol{v}^{2}+12 \delta(131+148 \nu) \boldsymbol{v}^{2}\right)\right. \\
& \left.+(n S)\left(-1152 \delta \kappa_{-} \boldsymbol{v}^{2}+48 \kappa_{+}(24+37 \nu) \boldsymbol{v}^{2}+12(219+296 \nu) \boldsymbol{v}^{2}\right)\right] \\
& +\left(-3 \delta \kappa_{+}(165+38 \nu) \boldsymbol{v}^{2}-2 \delta(-299+114 \nu) \boldsymbol{v}^{2}-3 \kappa_{-}(-165+292 \nu) \boldsymbol{v}^{2}\right)(v \Sigma) \\
& +(n v)^{2}\left[\left(-903 \delta \kappa_{-}+156(5+4 \nu)+3 \kappa_{+}(301+104 \nu)\right)(v S)\right. \\
& \left.\left.+\left(6 \delta(-185+52 \nu)+3 \delta \kappa_{+}(301+52 \nu)+3 \kappa_{-}(-301+550 \nu)\right)(v \Sigma)\right]\right\} \Sigma^{i} \\
& +\boldsymbol{v}^{i}\left(\left(-888 \delta-588 \kappa_{-}-444 \delta \kappa_{+}\right)(v S)^{2}+S^{2}\left(-1248 \delta \boldsymbol{v}^{2}-120 \kappa_{-} \boldsymbol{v}^{2}-624 \delta \kappa_{+} \boldsymbol{v}^{2}\right)\right.
\end{aligned}
$$




$$
\begin{aligned}
& +(n S)^{2}\left(4404 \delta \boldsymbol{v}^{2}-156 \kappa_{-} \boldsymbol{v}^{2}+2202 \delta \kappa_{+} \boldsymbol{v}^{2}\right)+(S \Sigma)\left(504 \delta \kappa_{-} \boldsymbol{v}^{2}+24 \kappa_{+}(-21+104 \nu) \boldsymbol{v}^{2}+4(-449+1248 \nu) \boldsymbol{v}^{2}\right) \\
& +(n \Sigma)^{2}\left(-3 \delta \kappa_{+}(-393+734 \nu) \boldsymbol{v}^{2}-6 \delta(-71+734 \nu) \boldsymbol{v}^{2}+3 \kappa_{-}(-393+1520 \nu) \boldsymbol{v}^{2}\right) \\
& +(n S)(n \Sigma)\left(-2358 \delta \kappa_{-} \boldsymbol{v}^{2}-6 \kappa_{+}(-393+1468 \nu) \boldsymbol{v}^{2}-6(-875+2936 \nu) \boldsymbol{v}^{2}\right) \\
& +\left(-144 \delta \kappa_{-}+48 \kappa_{+}(3+37 \nu)+2(-953+1776 \nu)\right)(v S)(v \Sigma) \\
& +\left(-12 \kappa_{-}(6+25 \nu)+12 \delta \kappa_{+}(6+37 \nu)+2 \delta(-257+444 \nu)\right)(v \Sigma)^{2} \\
& +(n v)\left\{\left[\left(5268 \delta+2190 \kappa_{-}+2634 \delta \kappa_{+}\right)(n S)+\left(-222 \delta \kappa_{-}-6 \kappa_{+}(-37+878 \nu)-6(-127+1756 \nu)\right)(n \Sigma)\right](v S)\right. \\
& +\left[\left(-222 \delta \kappa_{-}-24(-93+439 \nu)-6 \kappa_{+}(-37+878 \nu)\right)(n S)\right. \\
& \left.\left.+\left(-6 \delta \kappa_{+}(-37+439 \nu)+6 \kappa_{-}(-37+513 \nu)-6 \delta(183+878 \nu)\right)(n \Sigma)\right](v \Sigma)\right\} \\
& +\left(12 \delta \kappa_{+}(-21+52 \nu) \boldsymbol{v}^{2}-12 \kappa_{-}(-21+94 \nu) \boldsymbol{v}^{2}+8 \delta(-37+156 \nu) \boldsymbol{v}^{2}\right) \boldsymbol{\Sigma}^{2} \\
& +(n v)^{2}\left[\left(-8988 \delta-1632 \kappa_{-}-4494 \delta \kappa_{+}\right)(n S)^{2}+\left(2862 \delta \kappa_{-}\right.\right. \\
& \left.+6 \kappa_{+}(-477+2996 \nu)+6(-989+5992 \nu)\right)(n S)(n \Sigma) \\
& +\left(3 \delta \kappa_{+}(-477+1498 \nu)+6 \delta(243+1498 \nu)-3 \kappa_{-}(-477+2452 \nu)\right)(n \Sigma)^{2} \\
& +\left(1344 \delta+468 \kappa_{-}+672 \delta \kappa_{+}\right) S^{2}+\left(-204 \delta \kappa_{-}-12 \kappa_{+}(-17+224 \nu)-12(-211+448 \nu)\right)(S \Sigma) \\
& \left.\left.+\left(-24 \delta(-25+56 \nu)-6 \delta \kappa_{+}(-17+112 \nu)+6 \kappa_{-}(-17+146 \nu)\right) \boldsymbol{\Sigma}^{2}\right]\right) \\
& +\boldsymbol{n}^{i}\left(( v S ) \left[(n S)\left(1992 \delta \boldsymbol{v}^{2}-564 \kappa_{-} \boldsymbol{v}^{2}+996 \delta \kappa_{+} \boldsymbol{v}^{2}\right)\right.\right. \\
& \left.+(n \Sigma)\left(-780 \delta \kappa_{-} \boldsymbol{v}^{2}-24(-89+166 \nu) \boldsymbol{v}^{2}-12 \kappa_{+}(-65+166 \nu) \boldsymbol{v}^{2}\right)\right] \\
& +\left[(n \Sigma)\left(-12 \delta \kappa_{+}(-65+83 \nu) \boldsymbol{v}^{2}-12 \delta(69+166 \nu) \boldsymbol{v}^{2}+12 \kappa_{-}(-65+213 \nu) \boldsymbol{v}^{2}\right)\right. \\
& \left.+(n S)\left(-780 \delta \kappa_{-} \boldsymbol{v}^{2}-12 \kappa_{+}(-65+166 \nu) \boldsymbol{v}^{2}-12(151+332 \nu) \boldsymbol{v}^{2}\right)\right](v \Sigma) \\
& +(n v)^{2}\left\{\left[\left(-8472 \delta-2172 \kappa_{-}-4236 \delta \kappa_{+}\right)(n S)+\left(1032 \delta \kappa_{-}+24 \kappa_{+}(-43+353 \nu)\right.\right.\right. \\
& +6(-1023+2824 \nu))(n \Sigma)](v S)+\left[\left(1032 \delta \kappa_{-}+24 \kappa_{+}(-43+353 \nu)+12(-151+1412 \nu)\right)(n S)\right. \\
& \left.\left.+\left(12 \delta \kappa_{+}(-86+353 \nu)-12 \kappa_{-}(-86+525 \nu)+6 \delta(31+1412 \nu)\right)(n \Sigma)\right](v \Sigma)\right\} \\
& +(n v)^{3}\left[\left(12600 \delta-1230 \kappa_{-}+6300 \delta \kappa_{+}\right)(n S)^{2}+\left(-7530 \delta \kappa_{-}-90(-143+560 \nu)\right.\right. \\
& \left.-30 \kappa_{+}(-251+840 \nu)\right)(n S)(n \Sigma)+\left(-90 \delta(-3+140 \nu)-15 \delta \kappa_{+}(-251+420 \nu)\right. \\
& \left.+15 \kappa_{-}(-251+922 \nu)\right)(n \Sigma)^{2}+\left(-2016 \delta-210 \kappa_{-}-1008 \delta \kappa_{+}\right) S^{2} \\
& +\left(798 \delta \kappa_{-}+42 \kappa_{+}(-19+96 \nu)+48(-25+168 \nu)\right)(S \Sigma) \\
& \left.+\left(21 \delta \kappa_{+}(-19+48 \nu)+36 \delta(11+56 \nu)-21 \kappa_{-}(-19+86 \nu)\right) \boldsymbol{\Sigma}^{2}\right] \\
& +(n v)\left[\left(1464 \delta+1200 \kappa_{-}+732 \delta \kappa_{+}\right)(v S)^{2}+(n S)^{2}\left(-7512 \delta \boldsymbol{v}^{2}+2934 \kappa_{-} \boldsymbol{v}^{2}\right.\right. \\
& \left.-3756 \delta \kappa_{+} v^{2}\right)+S^{2}\left(1944 \delta v^{2}-126 \kappa_{-} v^{2}+972 \delta \kappa_{+} v^{2}\right)+(S \Sigma)\left(-1098 \delta \kappa_{-} v^{2}\right. \\
& \left.-72(-7+108 \nu) \boldsymbol{v}^{2}-18 \kappa_{+}(-61+216 \nu) \boldsymbol{v}^{2}\right)+(n \Sigma)^{2}\left(3 \delta \kappa_{+}(-1115+1252 \nu) \boldsymbol{v}^{2}\right. \\
& \left.+6 \delta(-233+1252 \nu) v^{2}-3 \kappa_{-}(-1115+3482 \nu) v^{2}\right) \\
& +(n S)(n \Sigma)\left(6690 \delta \kappa_{-} v^{2}+6 \kappa_{+}(-1115+2504 \nu) v^{2}+6(-1709+5008 \nu) \boldsymbol{v}^{2}\right) \\
& +\left(468 \delta \kappa_{-}-12 \kappa_{+}(39+244 \nu)-6(-751+976 \nu)\right)(v S)(v \Sigma)+\left(6 \kappa_{-}(39+44 \nu)-6 \delta \kappa_{+}(39+122 \nu)\right. \\
& \left.\left.-6 \delta(-255+244 \nu))(v \Sigma)^{2}+\left(-36 \delta(19+54 \nu) \boldsymbol{v}^{2}-9 \delta \kappa_{+}(-61+108 \nu) \boldsymbol{v}^{2}+9 \kappa_{-}(-61+230 \nu) \boldsymbol{v}^{2}\right) \boldsymbol{\Sigma}^{2}\right]\right) \text {, }
\end{aligned}
$$




$$
\begin{aligned}
h_{6}^{1 i}= & S^{i}\left\{(n v)\left[\left(60 \delta+32 \kappa_{-}+30 \delta \kappa_{+}\right)(n S)+\left(\delta \kappa_{-}+\kappa_{+}(-1-60 \nu)-5(1+24 \nu)\right)(n \Sigma)\right]\right. \\
& \left.+\left(-12 \delta-44 \kappa_{-}-6 \delta \kappa_{+}\right)(v S)+\left(59-19 \delta \kappa_{-}+24 \nu+\kappa_{+}(19+12 \nu)\right)(v \Sigma)\right\} \\
& +\left\{( n v ) \left[\left(79+\delta \kappa_{-}+\kappa_{+}(-1-60 \nu)-120 \nu\right)(n S)+\left(\kappa_{-}(1+28 \nu)-\delta \kappa_{+}(1+30 \nu)\right.\right.\right. \\
& -\delta(7+60 \nu))(n \Sigma)]+\left(-67-19 \delta \kappa_{-}+24 \nu+\kappa_{+}(19+12 \nu)\right)(v S) \\
& \left.+\left(-3 \delta^{3}+\delta \kappa_{+}(19+6 \nu)+\kappa_{-}(-19+32 \nu)\right)(v \Sigma)\right\} \boldsymbol{\Sigma}^{i} \\
& +\boldsymbol{v}^{i}\left[\left(192 \delta-16 \kappa_{-}+96 \delta \kappa_{+}\right)(n S)^{2}+\left(-112 \delta \kappa_{-}-16 \kappa_{+}(-7+24 \nu)-4(-49+192 \nu)\right)(n S)(n \Sigma)\right. \\
& +\left(-8 \delta \kappa_{+}(-7+12 \nu)+8 \kappa_{-}(-7+26 \nu)-3 \delta(1+64 \nu)\right)(n \Sigma)^{2}+\left(-60 \delta+20 \kappa_{-}-30 \delta \kappa_{+}\right) S^{2} \\
& +\left(50 \delta \kappa_{-}+10 \kappa_{+}(-5+12 \nu)+5(-13+48 \nu)\right)(S \Sigma) \\
& \left.+\left(5 \delta \kappa_{+}(-5+6 \nu)-5 \kappa_{-}(-5+16 \nu)+2 \delta(1+30 \nu)\right) \boldsymbol{\Sigma}^{2}\right]+\boldsymbol{n}^{i}\left\{\left[\left(24 \delta+52 \kappa_{-}+12 \delta \kappa_{+}\right)(n S)\right.\right. \\
& \left.+\left(20 \delta \kappa_{-}-4 \kappa_{+}(5+6 \nu)-3(-17+16 \nu)\right)(n \Sigma)\right](v S)+\left[\left(20 \delta \kappa_{-}-4 \kappa_{+}(5+6 \nu)-2(13+24 \nu)\right)(n S)\right. \\
& \left.+\left(-4 \delta \kappa_{+}(5+3 \nu)-4 \kappa_{-}(-5+7 \nu)-\delta(-1+24 \nu)\right)(n \Sigma)\right](v \Sigma)+(n v)\left[\left(-282 \delta-27 \kappa_{-}-141 \delta \kappa_{+}\right)(n S)^{2}\right. \\
& +\left(114 \delta \kappa_{-}+6 \kappa_{+}(-19+94 \nu)+3(-99+376 \nu)\right)(n S)(n \Sigma) \\
& +\left(3 \delta \kappa_{+}(-19+47 \nu)+6 \delta(1+47 \nu)-3 \kappa_{-}(-19+85 \nu)\right)(n \Sigma)^{2}+\left(66 \delta-19 \kappa_{-}+33 \delta \kappa_{+}\right) S^{2} \\
& \left.\left.+\left(59-52 \delta \kappa_{-}-264 \nu-4 \kappa_{+}(-13+33 \nu)\right)(S \Sigma)+\left(-66 \delta \nu-\delta \kappa_{+}(-26+33 \nu)+\kappa_{-}(-26+85 \nu)\right) \Sigma^{2}\right]\right\},
\end{aligned}
$$

$$
\begin{aligned}
& k_{5}^{0 i}=(\boldsymbol{v} \times \boldsymbol{S})^{i}\left(-2475 \delta(n v)^{4}+3726 \delta(n v)^{2} \boldsymbol{v}^{2}-1135 \delta\left(\boldsymbol{v}^{2}\right)^{2}\right) \\
& +(\boldsymbol{v} \times \boldsymbol{\Sigma})^{i}\left(75(-17+71 \nu)(n v)^{4}-6(-313+1315 \nu)(n v)^{2} \boldsymbol{v}^{2}+(-559+2325 \nu)\left(\boldsymbol{v}^{2}\right)^{2}\right) \\
& +(\boldsymbol{n} \times \boldsymbol{S})^{i}\left(3675 \delta(n v)^{5}-6210 \delta(n v)^{3} \boldsymbol{v}^{2}+2451 \delta(n v)\left(\boldsymbol{v}^{2}\right)^{2}\right) \\
& +(\boldsymbol{n} \times \boldsymbol{\Sigma})^{i}\left(-525(-3+13 \nu)(n v)^{5}+30(-87+379 \nu)(n v)^{3} \boldsymbol{v}^{2}-3(-341+1483 \nu)(n v)\left(\boldsymbol{v}^{2}\right)^{2}\right) \\
& +\boldsymbol{v}^{i}\left[(n v)^{3}(-1680 \delta(\operatorname{Snv})+60(-14+47 \nu)(\Sigma n v))+(n v)\left(1716 \delta(S n v) \boldsymbol{v}^{2}-12(-73+253 \nu) \boldsymbol{v}^{2}(\Sigma n v)\right)\right] \\
& +\boldsymbol{n}^{i}\left[1878 \delta(\operatorname{Snv})\left(\boldsymbol{v}^{2}\right)^{2}-6(-167+645 \nu)\left(\boldsymbol{v}^{2}\right)^{2}(\Sigma n v)+(n v)^{4}(5250 \delta(\operatorname{Snv})-1050(-3+11 \nu)(\Sigma n v))\right. \\
& \left.+(n v)^{2}\left(-7320 \delta(\operatorname{Snv}) \boldsymbol{v}^{2}+60(-70+261 \nu) \boldsymbol{v}^{2}\left(\sum n v\right)\right)\right], \\
& k_{5}^{1 i}=(\boldsymbol{v} \times \boldsymbol{S})^{i}\left(792 \delta(n v)^{2}-778 \delta \boldsymbol{v}^{2}\right)+(\boldsymbol{v} \times \mathbf{\Sigma})^{i}\left(-3(-136+567 \nu)(n v)^{2}+3(-134+567 \nu) \boldsymbol{v}^{2}\right) \\
& +(\boldsymbol{n} \times \boldsymbol{S})^{i}\left(-920 \delta(n v)^{3}+806 \delta(n v) \boldsymbol{v}^{2}\right)+(\boldsymbol{n} \times \boldsymbol{\Sigma})^{i}\left((-404+1763 \nu)(n v)^{3}-(-354+1559 \nu)(n v) \boldsymbol{v}^{2}\right) \\
& +(\boldsymbol{n} \times \boldsymbol{v})^{i}\left[(n v)^{2}(-16 \delta(n S)-2(-7+13 \nu)(n \Sigma))+12 \delta(n S) \boldsymbol{v}^{2}+2(-7+15 \nu)(n \Sigma) \boldsymbol{v}^{2}\right. \\
& +(n v)(4 \delta(v S)-4 \nu(v \Sigma))]+(n v) \boldsymbol{v}^{i}(-48 \delta(S n v)+4(-21+116 \nu)(\Sigma n v)) \\
& +\boldsymbol{n}^{i}\left[2025 \delta(S n v) \boldsymbol{v}^{2}-5(-175+662 \nu) \boldsymbol{v}^{2}\left(\sum n v\right)+(n v)^{2}(-1955 \delta(S n v)+(-833+2978 \nu)(\Sigma n v))\right] \text {, } \\
& k_{5}^{2 i}=59 \delta(\boldsymbol{n} \times \boldsymbol{S})^{i}(n v)+(21-95 \nu)(\boldsymbol{n} \times \boldsymbol{\Sigma})^{i}(n v)+(\boldsymbol{n} \times \boldsymbol{v})^{i}(\delta(n S)-\nu(n \Sigma))-35 \delta(\boldsymbol{v} \times \boldsymbol{S})^{i} \\
& +5(-3+13 \nu)(\boldsymbol{v} \times \Sigma)^{i}+\boldsymbol{n}^{i}(115 \delta(\operatorname{Snv})-2(-12+41 \nu)(\Sigma n v)), \\
& k_{6}^{0 i}=S^{i}\left\{(n v)^{4}\left(\left(-2100 \delta-1050 \delta \kappa_{+}\right)(n S)+\left(-1050 \delta^{2}+525 \delta \kappa_{-}-525 \delta^{2} \kappa_{+}\right)(n \Sigma)\right)\right. \\
& +(n S)\left(-852 \delta\left(\boldsymbol{v}^{2}\right)^{2}-12 \kappa_{-}\left(\boldsymbol{v}^{2}\right)^{2}-426 \delta \kappa_{+}\left(\boldsymbol{v}^{2}\right)^{2}\right)+(n \Sigma)\left(-426 \delta^{2}\left(\boldsymbol{v}^{2}\right)^{2}+207 \delta \kappa_{-}\left(\boldsymbol{v}^{2}\right)^{2}\right. \\
& \left.+3 \kappa_{+}(-69+284 \nu)\left(v^{2}\right)^{2}\right)+(n v)^{2}\left[(n S)\left(3000 \delta v^{2}+60 \kappa_{-} v^{2}+1500 \delta \kappa_{+} v^{2}\right)\right. \\
& \left.+(n \Sigma)\left(1500 \delta^{2} \boldsymbol{v}^{2}-720 \delta \kappa_{-} \boldsymbol{v}^{2}-120 \kappa_{+}(-6+25 \nu) \boldsymbol{v}^{2}\right)\right] \\
& +(n v)^{3}\left[\left(600 \delta-300 \kappa_{-}+300 \delta \kappa_{+}\right)(v S)+\left(300 \delta^{2}-300 \delta \kappa_{-}-300 \kappa_{+}(-1+2 \nu)\right)(v \Sigma)\right] \\
& \left.+(n v)\left[(v S)\left(-576 \delta \boldsymbol{v}^{2}+156 \kappa_{-} \boldsymbol{v}^{2}-288 \delta \kappa_{+} \boldsymbol{v}^{2}\right)+\left(-288 \delta^{2} \boldsymbol{v}^{2}+222 \delta \kappa_{-} \boldsymbol{v}^{2}+6 \kappa_{+}(-37+96 \nu) \boldsymbol{v}^{2}\right)(v \Sigma)\right]\right\}
\end{aligned}
$$




$$
\begin{aligned}
& +\left\{(n v)^{4}\left[\left(-1050 \delta^{2}+525 \delta \kappa_{-}-525 \delta^{2} \kappa_{+}\right)(n S)+\left(525 \delta^{2} \kappa_{-}+2100 \delta \nu+525 \delta \kappa_{+}(-1+2 \nu)\right)(n \Sigma)\right]\right. \\
& +(n \Sigma)\left(852 \delta \nu\left(\boldsymbol{v}^{2}\right)^{2}+3 \delta \kappa_{+}(-69+142 \nu)\left(\boldsymbol{v}^{2}\right)^{2}-3 \kappa_{-}(-69+280 \nu)\left(\boldsymbol{v}^{2}\right)^{2}\right)+(n S)\left(-426 \delta^{2}\left(\boldsymbol{v}^{2}\right)^{2}+207 \delta \kappa_{-}\left(\boldsymbol{v}^{2}\right)^{2}\right. \\
& \left.+3 \kappa_{+}(-69+284 \nu)\left(v^{2}\right)^{2}\right)+(n v)^{2}\left[(n S)\left(1500 \delta^{2} v^{2}-720 \delta \kappa_{-} v^{2}-120 \kappa_{+}(-6+25 \nu) v^{2}\right)\right. \\
& \left.+(n \Sigma)\left(-3000 \delta \nu v^{2}-60 \delta \kappa_{+}(-12+25 \nu) \boldsymbol{v}^{2}+60 \kappa_{-}(-12+49 \nu) \boldsymbol{v}^{2}\right)\right] \\
& +(n v)^{3}\left[\left(300 \delta^{2}-300 \delta \kappa_{-}-300 \kappa_{+}(-1+2 \nu)\right)(v S)+\left(-300 \delta \kappa_{+}(-1+\nu)\right.\right. \\
& \left.\left.-600 \delta \nu+300 \kappa_{-}(-1+3 \nu)\right)(v \Sigma)\right]+(n v)\left[( v S ) \left(-288 \delta^{2} \boldsymbol{v}^{2}+222 \delta \kappa_{-} v^{2}\right.\right. \\
& \left.\left.\left.+6 \kappa_{+}(-37+96 \nu) \boldsymbol{v}^{2}\right)+\left(576 \delta \nu \boldsymbol{v}^{2}+6 \delta \kappa_{+}(-37+48 \nu) \boldsymbol{v}^{2}-6 \kappa_{-}(-37+122 \nu) \boldsymbol{v}^{2}\right)(v \Sigma)\right]\right\} \boldsymbol{\Sigma}^{i} \\
& +\boldsymbol{v}^{i}\left(( v S ) \left[(n S)\left(-984 \delta \boldsymbol{v}^{2}-24 \kappa_{-} \boldsymbol{v}^{2}-492 \delta \kappa_{+} \boldsymbol{v}^{2}\right)+(n \Sigma)\left(-492 \delta^{2} \boldsymbol{v}^{2}\right.\right.\right. \\
& \left.\left.+234 \delta \kappa_{-} \boldsymbol{v}^{2}+6 \kappa_{+}(-39+164 \nu) \boldsymbol{v}^{2}\right)\right]+\left[( n \Sigma ) \left(984 \delta \nu \boldsymbol{v}^{2}\right.\right. \\
& \left.+6 \delta \kappa_{+}(-39+82 \nu) \boldsymbol{v}^{2}-6 \kappa_{-}(-39+160 \nu) \boldsymbol{v}^{2}\right)+(n S)\left(-492 \delta^{2} \boldsymbol{v}^{2}+234 \delta \kappa_{-} \boldsymbol{v}^{2}\right. \\
& \left.\left.+6 \kappa_{+}(-39+164 \nu) v^{2}\right)\right](v \Sigma)+(n v)^{2}\left\{\left[\left(2880 \delta+120 \kappa_{-}+1440 \delta \kappa_{+}\right)(n S)\right.\right. \\
& \left.+\left(1440 \delta^{2}-660 \delta \kappa_{-}-60 \kappa_{+}(-11+48 \nu)\right)(n \Sigma)\right](v S)+\left[\left(1440 \delta^{2}-660 \delta \kappa_{-}-60 \kappa_{+}(-11+48 \nu)\right)(n S)\right. \\
& \left.\left.+\left(-2880 \delta \nu-60 \delta \kappa_{+}(-11+24 \nu)+60 \kappa_{-}(-11+46 \nu)\right)(n \Sigma)\right](v \Sigma)\right\} \\
& +(n v)^{3}\left[\left(-5460 \delta-2730 \delta \kappa_{+}\right)(n S)^{2}+\left(-5460 \delta^{2}+2730 \delta \kappa_{-}-2730 \delta^{2} \kappa_{+}\right)(n S)(n \Sigma)\right. \\
& +\left(1365 \delta^{2} \kappa_{-}+5460 \delta \nu+1365 \delta \kappa_{+}(-1+2 \nu)\right)(n \Sigma)^{2}+\left(660 \delta+60 \kappa_{-}+330 \delta \kappa_{+}\right) S^{2} \\
& +\left(660 \delta^{2}-270 \delta \kappa_{-}-30 \kappa_{+}(-9+44 \nu)\right)(S \Sigma)+(-660 \delta \nu \\
& \left.\left.-15 \delta \kappa_{+}(-9+22 \nu)+15 \kappa_{-}(-9+40 \nu)\right) \boldsymbol{\Sigma}^{2}\right]+(n v)\left[\left(-336 \delta+12 \kappa_{-}-168 \delta \kappa_{+}\right)(v S)^{2}\right. \\
& +S^{2}\left(-708 \delta v^{2}-48 \kappa_{-} v^{2}-354 \delta \kappa_{+} v^{2}\right)+(n S)^{2}\left(4020 \delta v^{2}+2010 \delta \kappa_{+} v^{2}\right) \\
& +(n S)(n \Sigma)\left(4020 \delta^{2} \boldsymbol{v}^{2}-2010 \delta \kappa_{-} \boldsymbol{v}^{2}+2010 \delta^{2} \kappa_{+} \boldsymbol{v}^{2}\right)+(n \Sigma)^{2}\left(-1005 \delta^{2} \kappa_{-} \boldsymbol{v}^{2}\right. \\
& \left.-4020 \delta \nu v^{2}-1005 \delta \kappa_{+}(-1+2 \nu) v^{2}\right)+(S \Sigma)\left(-708 \delta^{2} v^{2}+306 \delta \kappa_{-} v^{2}+6 \kappa_{+}(-51+236 \nu) v^{2}\right) \\
& +\left(-336 \delta^{2}+180 \delta \kappa_{-}+12 \kappa_{+}(-15+56 \nu)\right)(v S)(v \Sigma) \\
& +\left(336 \delta \nu+6 \delta \kappa_{+}(-15+28 \nu)-6 \kappa_{-}(-15+58 \nu)\right)(v \Sigma)^{2} \\
& \left.\left.+\left(708 \delta \nu \boldsymbol{v}^{2}+3 \delta \kappa_{+}(-51+118 \nu) \boldsymbol{v}^{2}-3 \kappa_{-}(-51+220 \nu) \boldsymbol{v}^{2}\right) \boldsymbol{\Sigma}^{2}\right]\right) \\
& +n^{i}\left((v S)^{2}\left(-636 \delta v^{2}+30 \kappa_{-} v^{2}-318 \delta \kappa_{+} v^{2}\right)+S^{2}\left(-714 \delta\left(v^{2}\right)^{2}-6 \kappa_{-}\left(v^{2}\right)^{2}\right.\right. \\
& \left.-357 \delta \kappa_{+}\left(\boldsymbol{v}^{2}\right)^{2}\right)+(n S)^{2}\left(3630 \delta\left(\boldsymbol{v}^{2}\right)^{2}+1815 \delta \kappa_{+}\left(\boldsymbol{v}^{2}\right)^{2}\right)+(n S)(n \Sigma)\left(3630 \delta^{2}\left(\boldsymbol{v}^{2}\right)^{2}-1815 \delta \kappa_{-}\left(\boldsymbol{v}^{2}\right)^{2}\right. \\
& \left.+1815 \delta^{2} \kappa_{+}\left(v^{2}\right)^{2}\right)+(n \Sigma)^{2}\left(-\frac{1815}{2} \delta^{2} \kappa_{-}\left(v^{2}\right)^{2}-3630 \delta \nu\left(v^{2}\right)^{2}\right. \\
& \left.-\frac{1815}{2} \delta \kappa_{+}(-1+2 \nu)\left(v^{2}\right)^{2}\right)+(S \Sigma)\left(-714 \delta^{2}\left(v^{2}\right)^{2}+351 \delta \kappa_{-}\left(v^{2}\right)^{2}+3 \kappa_{+}(-117+476 \nu)\left(v^{2}\right)^{2}\right) \\
& +(v S)\left(-636 \delta^{2} \boldsymbol{v}^{2}+348 \delta \kappa_{-} \boldsymbol{v}^{2}+12 \kappa_{+}(-29+106 \nu) \boldsymbol{v}^{2}\right)(v \Sigma)+\left(636 \delta \nu \boldsymbol{v}^{2}\right. \\
& \left.+6 \delta \kappa_{+}(-29+53 \nu) \boldsymbol{v}^{2}-6 \kappa_{-}(-29+111 \nu) \boldsymbol{v}^{2}\right)(v \Sigma)^{2}+(n v)^{3}\left\{\left(\left(-16800 \delta-8400 \delta \kappa_{+}\right)(n S)\right.\right. \\
& \left.+\left(-8400 \delta^{2}+4200 \delta \kappa_{-}-4200 \delta^{2} \kappa_{+}\right)(n \Sigma)\right)(v S)+\left[\left(-8400 \delta^{2}+4200 \delta \kappa_{-}-4200 \delta^{2} \kappa_{+}\right)(n S)\right. \\
& \left.\left.+\left(4200 \delta^{2} \kappa_{-}+16800 \delta \nu+4200 \delta \kappa_{+}(-1+2 \nu)\right)(n \Sigma)\right](v \Sigma)\right\} \\
& +(n v)\left\{( v S ) \left((n S)\left(10680 \delta v^{2}+5340 \delta \kappa_{+} \boldsymbol{v}^{2}\right)+(n \Sigma)\left(5340 \delta^{2} \boldsymbol{v}^{2}-2670 \delta \kappa_{-} \boldsymbol{v}^{2}\right.\right.\right. \\
& \left.\left.+2670 \delta^{2} \kappa_{+} \boldsymbol{v}^{2}\right)\right)+\left[(n S)\left(5340 \delta^{2} \boldsymbol{v}^{2}-2670 \delta \kappa_{-} \boldsymbol{v}^{2}+2670 \delta^{2} \kappa_{+} \boldsymbol{v}^{2}\right)\right. \\
& \left.\left.+(n \Sigma)\left(-2670 \delta^{2} \kappa_{-} \boldsymbol{v}^{2}-10680 \delta \nu \boldsymbol{v}^{2}-2670 \delta \kappa_{+}(-1+2 \nu) \boldsymbol{v}^{2}\right)\right](v \Sigma)\right\}
\end{aligned}
$$




$$
\begin{aligned}
& +\left(714 \delta \nu\left(\boldsymbol{v}^{2}\right)^{2}+\frac{3}{2} \delta \kappa_{+}(-117+238 \nu)\left(\boldsymbol{v}^{2}\right)^{2}-\frac{3}{2} \kappa_{-}(-117+472 \nu)\left(\boldsymbol{v}^{2}\right)^{2}\right) \boldsymbol{\Sigma}^{2} \\
& +(n v)^{4}\left[\left(28350 \delta+14175 \delta \kappa_{+}\right)(n S)^{2}+\left(28350 \delta^{2}-14175 \delta \kappa_{-}+14175 \delta^{2} \kappa_{+}\right)(n S)(n \Sigma)\right. \\
& +\left(-\frac{14175}{2} \delta^{2} \kappa_{-}-28350 \delta \nu-\frac{14175}{2} \delta \kappa_{+}(-1+2 \nu)\right)(n \Sigma)^{2}+\left(-3150 \delta-1575 \delta \kappa_{+}\right) S^{2} \\
& \left.+\left(-3150 \delta^{2}+1575 \delta \kappa_{-}-1575 \delta^{2} \kappa_{+}\right)(S \Sigma)+\left(\frac{1575}{2} \delta^{2} \kappa_{-}+3150 \delta \nu+\frac{1575}{2} \delta \kappa_{+}(-1+2 \nu)\right) \Sigma^{2}\right] \\
& +(n v)^{2}\left[\left(2100 \delta-150 \kappa_{-}+1050 \delta \kappa_{+}\right)(v S)^{2}\right. \\
& +(n S)^{2}\left(-27300 \delta v^{2}-13650 \delta \kappa_{+} \boldsymbol{v}^{2}\right)+S^{2}\left(3840 \delta v^{2}+30 \kappa_{-} v^{2}+1920 \delta \kappa_{+} \boldsymbol{v}^{2}\right) \\
& +(n S)(n \Sigma)\left(-27300 \delta^{2} \boldsymbol{v}^{2}+13650 \delta \kappa_{-} \boldsymbol{v}^{2}-13650 \delta^{2} \kappa_{+} \boldsymbol{v}^{2}\right)+(n \Sigma)^{2}\left(6825 \delta^{2} \kappa_{-} \boldsymbol{v}^{2}\right. \\
& \left.+27300 \delta \nu v^{2}+6825 \delta \kappa_{+}(-1+2 \nu) \boldsymbol{v}^{2}\right)+(S \Sigma)\left(3840 \delta^{2} \boldsymbol{v}^{2}-1890 \delta \kappa_{-} \boldsymbol{v}^{2}\right. \\
& \left.-30 \kappa_{+}(-63+256 \nu) \boldsymbol{v}^{2}\right)+\left(2100 \delta^{2}-1200 \delta \kappa_{-}-600 \kappa_{+}(-2+7 \nu)\right)(v S)(v \Sigma) \\
& +\left(-2100 \delta \nu-150 \delta \kappa_{+}(-4+7 \nu)+150 \kappa_{-}(-4+15 \nu)\right)(v \Sigma)^{2}+\left(-3840 \delta \nu v^{2}\right. \\
& \left.\left.\left.-15 \delta \kappa_{+}(-63+128 \nu) \boldsymbol{v}^{2}+15 \kappa_{-}(-63+254 \nu) \boldsymbol{v}^{2}\right) \boldsymbol{\Sigma}^{2}\right]\right)
\end{aligned}
$$

$$
\begin{aligned}
& k_{6}^{1 i}=\boldsymbol{S}^{i}\left\{(n v)^{2}\left[\left(1212 \delta+108 \kappa_{-}+606 \delta \kappa_{+}\right)(n S)+\left(-249 \delta \kappa_{-}-6(-121+404 \nu)-3 \kappa_{+}(-83+404 \nu)\right)(n \Sigma)\right]\right. \\
& +(n S)\left(-1092 \delta v^{2}-80 \kappa_{-} v^{2}-546 \delta \kappa_{+} v^{2}\right)+(n \Sigma)\left(233 \delta \kappa_{-} v^{2}+26(-25+84 \nu) \boldsymbol{v}^{2}\right. \\
& \left.\left.+\kappa_{+}(-233+1092 \nu) \boldsymbol{v}^{2}\right)+(n v)\left(68 \kappa_{-}(v S)+\left(-16+34 \delta \kappa_{-}-34 \kappa_{+}\right)(v \Sigma)\right)\right\} \\
& +\left\{( n v ) ^ { 2 } \left[\left(-249 \delta \kappa_{-}-6(-93+404 \nu)-3 \kappa_{+}(-83+404 \nu)\right)(n S)+(-12 \delta(-6+101 \nu)\right.\right. \\
& \left.\left.-3 \delta \kappa_{+}(-83+202 \nu)+3 \kappa_{-}(-83+368 \nu)\right)(n \Sigma)\right]+(n \Sigma)\left(4 \delta(-17+273 \nu) \boldsymbol{v}^{2}+\delta \kappa_{+}(-233+546 \nu) \boldsymbol{v}^{2}\right. \\
& \left.-\kappa_{-}(-233+1012 \nu) \boldsymbol{v}^{2}\right)+(n S)\left(233 \delta \kappa_{-} \boldsymbol{v}^{2}+6(-85+364 \nu) \boldsymbol{v}^{2}+\kappa_{+}(-233+1092 \nu) \boldsymbol{v}^{2}\right) \\
& \left.+(n v)\left[\left(12+34 \delta \kappa_{-}-34 \kappa_{+}\right)(v S)+\left(-4 \delta-34 \delta \kappa_{+}-34 \kappa_{-}(-1+2 \nu)\right)(v \Sigma)\right]\right\} \Sigma^{i} \\
& +\mathbf{v}^{i}\left\{\left[\left(-480 \delta-8 \kappa_{-}-240 \delta \kappa_{+}\right)(n S)+\left(116 \delta \kappa_{-}+8(-37+120 \nu)+4 \kappa_{+}(-29+120 \nu)\right)(n \Sigma)\right](v S)\right. \\
& +\left[\left(116 \delta \kappa_{-}+64(-2+15 \nu)+4 \kappa_{+}(-29+120 \nu)\right)(n S)+\left(4 \delta \kappa_{+}(-29+60 \nu)\right.\right. \\
& \left.\left.+8 \delta(7+60 \nu)-4 \kappa_{-}(-29+118 \nu)\right)(n \Sigma)\right](v \Sigma)+(n v)\left[\left(-168 \delta-78 \kappa_{-}-84 \delta \kappa_{+}\right)(n S)^{2}\right. \\
& +\left(6 \delta \kappa_{-}+12(-19+56 \nu)+6 \kappa_{+}(-1+56 \nu)\right)(n S)(n \Sigma)+\left(12 \delta(-5+14 \nu)+3 \delta \kappa_{+}(-1+28 \nu)\right. \\
& \left.-3 \kappa_{-}(-1+30 \nu)\right)(n \Sigma)^{2}+\left(216 \delta+6 \kappa_{-}+108 \delta \kappa_{+}\right) S^{2} \\
& +\left(-102 \delta \kappa_{-}-6 \kappa_{+}(-17+72 \nu)-8(-25+108 \nu)\right)(S \Sigma) \\
& \left.\left.+\left(-8 \delta(2+27 \nu)-3 \delta \kappa_{+}(-17+36 \nu)+3 \kappa_{-}(-17+70 \nu)\right) \Sigma^{2}\right]\right\} \\
& +\boldsymbol{n}^{i}\left(\left(-1176 \delta-4 \kappa_{-}-588 \delta \kappa_{+}\right)(v S)^{2}+\boldsymbol{S}^{2}\left(-2460 \delta \boldsymbol{v}^{2}+30 \kappa_{-} \boldsymbol{v}^{2}-1230 \delta \kappa_{+} \boldsymbol{v}^{2}\right)\right. \\
& +(n S)^{2}\left(9648 \delta v^{2}-6 \kappa_{-} v^{2}+4824 \delta \kappa_{+} v^{2}\right)+(S \Sigma)\left(1260 \delta \kappa_{-} v^{2}\right. \\
& \left.+60 \kappa_{+}(-21+82 \nu) \boldsymbol{v}^{2}+4(-641+2460 \nu) \boldsymbol{v}^{2}\right)+(n S)(n \Sigma)\left(-4830 \delta \kappa_{-} \boldsymbol{v}^{2}-6 \kappa_{+}(-805+3216 \nu) \boldsymbol{v}^{2}\right. \\
& \left.-12(-803+3216 \nu) \boldsymbol{v}^{2}\right)+(n \Sigma)^{2}\left(-12 \delta(1+804 \nu) \boldsymbol{v}^{2}-3 \delta \kappa_{+}(-805+1608 \nu) \boldsymbol{v}^{2}\right. \\
& \left.+3 \kappa_{-}(-805+3218 \nu) \boldsymbol{v}^{2}\right)+\left(584 \delta \kappa_{-}+112(-11+42 \nu)+8 \kappa_{+}(-73+294 \nu)\right)(v S)(v \Sigma) \\
& +\left(56 \delta(-1+21 \nu)+4 \delta \kappa_{+}(-73+147 \nu)-4 \kappa_{-}(-73+293 \nu)\right)(v \Sigma)^{2} \\
& +(n v)\left\{\left[\left(7080 \delta+96 \kappa_{-}+3540 \delta \kappa_{+}\right)(n S)+\left(-1722 \delta \kappa_{-}-48(-76+295 \nu)\right.\right.\right. \\
& \left.\left.-6 \kappa_{+}(-287+1180 \nu)\right)(n \Sigma)\right](v S)+\left[\left(-1722 \delta \kappa_{-}-120(-29+118 \nu)-6 \kappa_{+}(-287+1180 \nu)\right)(n S)\right.
\end{aligned}
$$




$$
\begin{aligned}
& \left.\left.+\left(-24 \delta(-2+295 \nu)-6 \delta \kappa_{+}(-287+590 \nu)+6 \kappa_{-}(-287+1164 \nu)\right)(n \Sigma)\right](v \Sigma)\right\} \\
& +\left(30 \delta \kappa_{+}(-21+41 \nu) \boldsymbol{v}^{2}-30 \kappa_{-}(-21+83 \nu) \boldsymbol{v}^{2}+4 \delta(-26+615 \nu) \boldsymbol{v}^{2}\right) \boldsymbol{\Sigma}^{2} \\
& +(n v)^{2}\left[\left(-16104 \delta+12 \kappa_{-}-8052 \delta \kappa_{+}\right)(n S)^{2}+\left(8064 \delta \kappa_{-}+48 \kappa_{+}(-168+671 \nu)\right.\right. \\
& +48(-335+1342 \nu))(n S)(n \Sigma)+\left(12 \delta \kappa_{+}(-336+671 \nu)+24 \delta(1+671 \nu)-12 \kappa_{-}(-336+1343 \nu)\right)(n \Sigma)^{2} \\
& +\left(2604 \delta-72 \kappa_{-}+1302 \delta \kappa_{+}\right) S^{2}+\left(-1374 \delta \kappa_{-}-6 \kappa_{+}(-229+868 \nu)-12(-227+868 \nu)\right)(S \Sigma) \\
& \left.\left.+\left(-12 \delta(-10+217 \nu)-3 \delta \kappa_{+}(-229+434 \nu)+3 \kappa_{-}(-229+892 \nu)\right) \boldsymbol{\Sigma}^{2}\right]\right), \\
& k_{6}^{2 i}=\left[\left(-288 \delta+32 \kappa_{-}-144 \delta \kappa_{+}\right)(n S)+\left(88 \delta \kappa_{-}+32(-5+18 \nu)+8 \kappa_{+}(-11+36 \nu)\right)(n \Sigma)\right] S^{i} \\
& \quad+\left[\left(88 \delta \kappa_{-}+8 \kappa_{+}(-11+36 \nu)+12(-11+48 \nu)\right)(n S)+\left(8 \delta \kappa_{+}(-11+18 \nu)-8 \kappa_{-}(-11+40 \nu)\right.\right. \\
& \quad+4 \delta(-1+72 \nu))(n \Sigma)] \boldsymbol{\Sigma}^{i} \\
& \quad+n^{i}\left[\left(1368 \delta+4 \kappa_{-}+684 \delta \kappa_{+}\right)(n S)^{2}+\left(-680 \delta \kappa_{-}-8 \kappa_{+}(-85+342 \nu)-4(-341+1368 \nu)\right)(n S)(n \Sigma)\right. \\
& \quad+\left(-4 \delta \kappa_{+}(-85+171 \nu)+4 \kappa_{-}(-85+341 \nu)-4 \delta(1+342 \nu)\right)(n \Sigma)^{2}+\left(-360 \delta-12 \kappa_{-}-180 \delta \kappa_{+}\right) S^{2} \\
& \quad+\left(168 \delta \kappa_{-}+24 \kappa_{+}(-7+30 \nu)+8(-47+180 \nu)\right)(S \Sigma)+\left(12 \delta \kappa_{+}(-7+15 \nu)\right. \\
& \left.\left.\quad-12 \kappa_{-}(-7+29 \nu)+8 \delta(-2+45 \nu)\right) \boldsymbol{\Sigma}^{2}\right] .
\end{aligned}
$$

[1] R. Abbott et al., Open data from the first and second observing runs of Advanced LIGO and Advanced Virgo, SoftwareX 13, 100658 (2021).

[2] R. Abbott et al., GWTC-2: Compact Binary Coalescences Observed by LIGO and Virgo During the First Half of the Third Observing Run, Phys. Rev. X 11, 021053 (2021).

[3] A. Buonanno and B. S. Sathyaprakash, Sources of gravitational waves: Theory and observations, arXiv:1410.7832.

[4] R. A. Porto, The tune of love and the nature(ness) of spacetime, Fortsch. Phys. 64, 723 (2016).

[5] R. A. Porto, The music of the spheres: The dawn of gravitational wave science, arXiv:1703.06440.

[6] A. Arvanitaki and S. Dubovsky, Exploring the string axiverse with precision black hole physics, Phys. Rev. D 83, 044026 (2011).

[7] A. Arvanitaki, M. Baryakhtar, S. Dimopoulos, S. Dubovsky, and R. Lasenby, Black hole mergers and the QCD axion at Advanced LIGO, Phys. Rev. D 95, 043001 (2017).

[8] R. Brito, S. Ghosh, E. Barausse, E. Berti, V. Cardoso, I. Dvorkin, A. Klein, and P. Pani, Stochastic and Resolvable Gravitational Waves from Ultralight Bosons, Phys. Rev. Lett. 119, 131101 (2017).

[9] K. K. Y. Ng, M. Isi, C.-J. Haster, and S. Vitale, Multiband gravitational-wave searches for ultralight bosons, Phys. Rev. D 102, 083020 (2020).

[10] K. K. Y. Ng, S. Vitale, O. A. Hannuksela, and T. G. F. Li, Constraints on Ultralight Scalar Bosons within Black Hole Spin Measurements from LIGO-Virgo's GWTC-2, Phys. Rev. Lett. 126, 151102 (2021).
[11] D. Baumann, H. S. Chia, and R. A. Porto, Probing ultralight bosons with binary black holes, Phys. Rev. D 99, 044001 (2019).

[12] D. Baumann, H. S. Chia, R. A. Porto, and J. Stout, Gravitational collider physics, Phys. Rev. D 101, 083019 (2020).

[13] P. Amaro-Seoane et al., Laser interferometer space antenna, arXiv:1702.00786.

[14] M. Punturo et al., The Einstein telescope: A thirdgeneration gravitational wave observatory, Classical Quantum Gravity 27, 194002 (2010).

[15] S. Vitale, R. Lynch, J. Veitch, V. Raymond, and R. Sturani, Measuring the Spin of Black Holes in Binary Systems Using Gravitational Waves, Phys. Rev. Lett. 112, 251101 (2014).

[16] B. Zackay, T. Venumadhav, L. Dai, J. Roulet, and M. Zaldarriaga, Highly spinning and aligned binary black hole merger in the Advanced LIGO first observing run, Phys. Rev. D 100, 023007 (2019).

[17] I. Hinder, S. Ossokine, H. P. Pfeiffer, and A. Buonanno, Gravitational waveforms for high spin and high massratio binary black holes: A synergistic use of numericalrelativity codes, Phys. Rev. D 99, 061501 (2019).

[18] L. Blanchet, Gravitational radiation from post-Newtonian sources and inspiralling compact binaries, Living Rev. Relativity 17, 2 (2014).

[19] G. Schäfer and P. Jaranowski, Hamiltonian formulation of general relativity and post-Newtonian dynamics of compact binaries, Living Rev. Relativity 21, 7 (2018).

[20] W. D. Goldberger, Course 7 - Effective field theories and gravitational radiation, in Les Houches Summer 
School-Session 86, edited by F. Bernardeau, C. Grojean, and J. Dalibard (Elsevier, NewYork, 2007), Vol. 86, pp. 351-396.

[21] R. A. Porto, The effective field theorist's approach to gravitational dynamics, Phys. Rep. 633, 1 (2016).

[22] L. Blanchet, T. Damour, and G. Esposito-Farese, Dimensional regularization of the third postNewtonian dynamics of point particles in harmonic coordinates, Phys. Rev. D 69, 124007 (2004).

[23] S. Foffa and R. Sturani, Effective field theory calculation of conservative binary dynamics at third post-Newtonian order, Phys. Rev. D 84, 044031 (2011).

[24] S. Foffa and R. Sturani, Dynamics of the gravitational twobody problem at fourth post-Newtonian order and at quadratic order in the Newton constant, Phys. Rev. D 87, 064011 (2013).

[25] C. R. Galley, A. K. Leibovich, R. A. Porto, and A. Ross, Tail effect in gravitational radiation reaction: Time nonlocality and renormalization group evolution, Phys. Rev. D 93, 124010 (2016).

[26] S. Foffa, P. Mastrolia, R. Sturani, and C. Sturm, Effective field theory approach to the gravitational two-body dynamics, at fourth post-Newtonian order and quintic in the Newton constant, Phys. Rev. D 95, 104009 (2017).

[27] R. A. Porto and I. Rothstein, Apparent ambiguities in the post-Newtonian expansion for binary systems, Phys. Rev. D 96, 024062 (2017).

[28] T. Damour, P. Jaranowski, and G. Schäfer, Nonlocal-intime action for the fourth post-Newtonian conservative dynamics of two-body systems, Phys. Rev. D 89, 064058 (2014).

[29] P. Jaranowski and G. Schäfer, Derivation of local-in-time fourth post-Newtonian ADM Hamiltonian for spinless compact binaries, Phys. Rev. D 92, 124043 (2015).

[30] L. Bernard, L. Blanchet, A. Bohe, G. Faye, and S. Marsat, Fokker action of nonspinning compact binaries at the fourth post-Newtonian approximation, Phys. Rev. D 93, 084037 (2016).

[31] L. Bernard, L. Blanchet, A. Bohe, G. Faye, and S. Marsat, Energy and periastron advance of compact binaries on circular orbits at the fourth post-Newtonian order, Phys. Rev. D 95, 044026 (2017).

[32] T. Marchand, L. Bernard, L. Blanchet, and G. Faye, Ambiguity-free completion of the equations of motion of compact binary systems at the fourth post-Newtonian order, Phys. Rev. D 97, 044023 (2018).

[33] S. Foffa and R. Sturani, Conservative dynamics of binary systems to fourth post-Newtonian order in the EFT approach I: Regularized Lagrangian, Phys. Rev. D 100, 024047 (2019).

[34] S. Foffa, R. A. Porto, I. Rothstein, and R. Sturani, Conservative dynamics of binary systems to fourth PostNewtonian order in the EFT approach II: Renormalized Lagrangian, Phys. Rev. D 100, 024048 (2019).

[35] S. Foffa, P. Mastrolia, R. Sturani, C. Sturm, and W. J. Torres Bobadilla, Static Two-Body Potential at Fifth PostNewtonian Order, Phys. Rev. Lett. 122, 241605 (2019).

[36] J. Blümlein, A. Maier, and P. Marquard, Five-loop static contribution to the gravitational interaction potential of two point masses, Phys. Lett. B 800, 135100 (2020).
[37] J. Blümlein, A. Maier, P. Marquard, and G. Schäfer, The fifth-order post-Newtonian Hamiltonian dynamics of two-body systems from an effective field theory approach: Potential contributions, Nucl. Phys. B965, 115352 (2021).

[38] J. Blümlein, A. Maier, P. Marquard, and G. Schäfer, Testing binary dynamics in gravity at the sixth postNewtonian level, Phys. Lett. B 807, 135496 (2020).

[39] J. Blümlein, A. Maier, P. Marquard, and G. Schäfer, The 6th post-Newtonian potential terms at $O\left(G_{N}^{4}\right)$, Phys. Lett. B 816, 136260 (2021).

[40] D. Bini, T. Damour, and A. Geralico, Sixth post-Newtonian local-in-time dynamics of binary systems, Phys. Rev. D 102, 024061 (2020).

[41] L. Blanchet, S. Foffa, F. Larrouturou, and R. Sturani, Logarithmic tail contributions to the energy function of circular compact binaries, Phys. Rev. D 101, 084045 (2020).

[42] G. Faye, L. Blanchet, and A. Buonanno, Higher-order spin effects in the dynamics of compact binaries. I. Equations of motion, Phys. Rev. D 74, 104033 (2006).

[43] R. A. Porto and I. Z. Rothstein, The Hyperfine EinsteinInfeld-Hoffmann Potential, Phys. Rev. Lett. 97, 021101 (2006).

[44] R. A. Porto, New results at 3PN via an effective field theory of gravity, in 11th Marcel Grossmann Meeting on General Relativity (World Scientific Publishing, 2007), pp. 2493-2496.

[45] R. A. Porto and I. Z. Rothstein, Spin(1)Spin(2) effects in the motion of inspiralling compact binaries at third order in the post-Newtonian expansion, Phys. Rev. D 78, 044012 (2008).

[46] R. A. Porto and I. Z. Rothstein, Next to leading order spin (1)spin(1) effects in the motion of inspiralling compact binaries, Phys. Rev. D 78, 044013 (2008).

[47] J. Steinhoff, S. Hergt, and G. Schaefer, On the next-toleading order gravitational spin(1)-spin(2) dynamics, Phys. Rev. D 77, 081501 (2008).

[48] J. Steinhoff, S. Hergt, and G. Schaefer, Spin-squared Hamiltonian of next-to-leading order gravitational interaction, Phys. Rev. D 78, 101503 (2008).

[49] R. A. Porto, Next-to-leading order spin-orbit effects in the motion of inspiralling compact binaries, Classical Quantum Gravity 27, 205001 (2010).

[50] M. Levi and J. Steinhoff, Next-to-next-to-leading order gravitational spin-orbit coupling via the effective field theory for spinning objects in the post-Newtonian scheme, J. Cosmol. Astropart. Phys. 01 (2016) 011.

[51] M. Levi and J. Steinhoff, Complete conservative dynamics for inspiralling compact binaries with spins at fourth postNewtonian order, arXiv:1607.04252.

[52] M. Levi, A. J. Mcleod, and M. Von Hippel, NNNLO gravitational quadratic-in-spin interactions at the quartic order in G, arXiv:2003.07890.

[53] M. Levi, A. J. Mcleod, and M. Von Hippel, $N^{3}$ LO gravitational spin-orbit coupling at order $G^{4}$, arXiv:2003.02827.

[54] A. Antonelli, C. Kavanagh, M. Khalil, J. Steinhoff, and J. Vines, Gravitational Spin-Orbit Coupling Through ThirdSubleading Post-Newtonian Order: From First-Order Self-Force to Arbitrary Mass Ratios, Phys. Rev. Lett. 125, 011103 (2020). 
[55] A. Antonelli, C. Kavanagh, M. Khalil, J. Steinhoff, and J. Vines, Gravitational spin-orbit and aligned $\operatorname{spin}_{1}-\operatorname{spin}_{2}$ couplings through third-subleading post-Newtonian orders, Phys. Rev. D 102, 124024 (2020).

[56] W. D. Goldberger and I. Z. Rothstein, An effective field theory of gravity for extended objects, Phys. Rev. D 73, 104029 (2006).

[57] R. A. Porto, Post-Newtonian corrections to the motion of spinning bodies in NRGR, Phys. Rev. D 73, 104031 (2006).

[58] H. Elvang and Y.-T. Huang, Scattering Amplitudes in Gauge Theory and Gravity, (Cambridge University Press, Cambridge, England, 2015).

[59] Z. Bern, J. J. Carrasco, M. Chiodaroli, H. Johansson, and R. Roiban, The duality between color and kinematics and its applications, arXiv:1909.01358.

[60] G. Kälin and R. A. Porto, From boundary data to bound states, J. High Energy Phys. 01 (2020) 072.

[61] G. Kälin and R. A. Porto, From boundary data to bound states. Part II. Scattering angle to dynamical invariants (with twist), J. High Energy Phys. 02 (2020) 120.

[62] G. Kälin and R. A. Porto, Post-Minkowskian effective field theory for conservative binary dynamics, J. High Energy Phys. 11 (2020) 106.

[63] C. Cheung, I. Z. Rothstein, and M. P. Solon, From Scattering Amplitudes to Classical Potentials in the PostMinkowskian Expansion, Phys. Rev. Lett. 121, 251101 (2018).

[64] D. A. Kosower, B. Maybee, and D. O'Connell, Amplitudes, observables, and classical scattering, J. High Energy Phys. 02 (2019) 137.

[65] Z. Bern, C. Cheung, R. Roiban, C.-H. Shen, M. P. Solon, and M. Zeng, Scattering Amplitudes and the Conservative Hamiltonian for Binary Systems at Third Post-Minkowskian Order, Phys. Rev. Lett. 122, 201603 (2019).

[66] Z. Bern, C. Cheung, R. Roiban, C.-H. Shen, M. P. Solon, and M. Zeng, Black hole binary dynamics from the double copy and effective theory, J. High Energy Phys. 10 (2019) 206.

[67] C. Cheung and M. P. Solon, Classical gravitational scattering at $\mathcal{O}\left(\mathrm{G}^{3}\right)$ from Feynman diagrams, J. High Energy Phys. 06 (2020) 144.

[68] C. Cheung and M.P. Solon, Tidal Effects in the PostMinkowskian Expansion, Phys. Rev. Lett. 125, 191601 (2020).

[69] G. Kälin, Z. Liu, and R. A. Porto, Conservative Dynamics of Binary Systems to Third Post-Minkowskian Order from the Effective Field Theory Approach, Phys. Rev. Lett. 125, 261103 (2020).

[70] G. Kälin, Z. Liu, and R. A. Porto, Conservative tidal effects in compact binary systems to next-to-leading postMinkowskian order, Phys. Rev. D 102, 124025 (2020).

[71] Z. Bern, J. Parra-Martinez, R. Roiban, E. Sawyer, and C.-H. Shen, Leading nonlinear tidal effects and scattering amplitudes, arXiv:2010.08559.

[72] C. Cheung, N. Shah, and M. P. Solon, Mining the geodesic equation for scattering data, Phys. Rev. D 103, 024030 (2021).

[73] Z. Bern, J. Parra-Martinez, R. Roiban, M. S. Ruf, C.-H. Shen, M. P. Solon, and M. Zeng, Scattering Amplitudes and Conservative Binary Dynamics at $\mathcal{O}\left(G^{4}\right)$, Phys. Rev. Lett. 126, 171601 (2021).

[74] J. Vines, Scattering of two spinning black holes in post-Minkowskian gravity, to all orders in spin, and effective-one-body mappings, Classical Quantum Gravity 35, 084002 (2018).

[75] J. Vines, J. Steinhoff, and A. Buonanno, Spinning-black-hole scattering and the test-black-hole limit at second postMinkowskian order, Phys. Rev. D 99, 064054 (2019).

[76] B. Maybee, D. O'Connell, and J. Vines, Observables and amplitudes for spinning particles and black holes, J. High Energy Phys. 12 (2019) 156.

[77] A. Guevara, A. Ochirov, and J. Vines, Scattering of spinning black holes from exponentiated soft factors, J. High Energy Phys. 09 (2019) 056.

[78] A. Guevara, A. Ochirov, and J. Vines, Black-hole scattering with general spin directions from minimal-coupling amplitudes, Phys. Rev. D 100, 104024 (2019).

[79] R. Aoude, K. Haddad, and A. Helset, Tidal effects for spinning particles, J. High Energy Phys. 03 (2021) 097.

[80] M.-Z. Chung, Y.-T. Huang, J.-W. Kim, and S. Lee, Complete Hamiltonian for spinning binary systems at first post-Minkowskian order, J. High Energy Phys. 05 (2020) 105.

[81] Z. Bern, A. Luna, R. Roiban, C.-H. Shen, and M. Zeng, Spinning black hole binary dynamics, scattering amplitudes and effective field theory, arXiv:2005.03071.

[82] D. Kosmopoulos and A. Luna, Quadratic-in-spin Hamiltonian at $\mathcal{O}\left(G^{2}\right)$ from scattering amplitudes, arXiv: 2102.10137.

[83] Z. Liu, R. A. Porto, and Z. Yang, Spin effects in the effective field theory approach to post-Minkowskian conservative dynamics, arXiv:2102.10059.

[84] E. Herrmann, J. Parra-Martinez, M. S. Ruf, and M. Zeng, Gravitational Bremsstrahlung from Reverse Unitarity, Phys. Rev. Lett. 126, 201602 (2021).

[85] P. Di Vecchia, C. Heissenberg, R. Russo, and G. Veneziano, Radiation reaction from soft theorems, arXiv: 2101.05772.

[86] G. Mogull, J. Plefka, and J. Steinhoff, Classical black hole scattering from a worldline quantum field theory, J. High Energy Phys. 02 (2021) 048.

[87] G. U. Jakobsen, G. Mogull, J. Plefka, and J. Steinhoff, Classical Gravitational Bremsstrahlung from a Worldline Quantum Field Theory, Phys. Rev. Lett. 126, 201103 (2021).

[88] S. Mougiakakos, M. M. Riva, and F. Vernizzi, Gravitational bremsstrahlung in the post-Minkowskian effective field theory, arXiv:2102.08339.

[89] L. Blanchet, T. Damour, G. Esposito-Farese, and B. R. Iyer, Gravitational Radiation from Inspiralling Compact Binaries Completed at the Third post-Newtonian Order, Phys. Rev. Lett. 93, 091101 (2004).

[90] T. Marchand, Q. Henry, F. Larrouturou, S. Marsat, G. Faye, and L. Blanchet, The mass quadrupole moment of compact binary systems at the fourth post-Newtonian order, Classical Quantum Gravity 37, 215006 (2020).

[91] L. Blanchet, A. Buonanno, and G. Faye, Higher-order spin effects in the dynamics of compact binaries. II. Radiation field, Phys. Rev. D 74, 104034 (2006); Erratum, Phys. Rev. 
D 75, 049903 (2007); Erratum, Phys. Rev. D 81, 089901 (2010).

[92] A. Bohé, S. Marsat, and L. Blanchet, Next-to-next-toleading order spin-orbit effects in the gravitational wave flux and orbital phasing of compact binaries, Classical Quantum Gravity 30, 135009 (2013).

[93] R. A. Porto, A. Ross, and I. Z. Rothstein, Spin induced multipole moments for the gravitational wave flux from binary inspirals to third Post-Newtonian order, J. Cosmol. Astropart. Phys. 03 (2011) 009.

[94] R. A. Porto, A. Ross, and I. Z. Rothstein, Spin induced multipole moments for the gravitational wave amplitude from binary inspirals to 2.5 Post-Newtonian order, J. Cosmol. Astropart. Phys. 09 (2012) 028.

[95] A. Bohé, G. Faye, S. Marsat, and E. K. Porter, Quadraticin-spin effects in the orbital dynamics and gravitationalwave energy flux of compact binaries at the 3PN order, Classical Quantum Gravity 32, 195010 (2015).

[96] N. T. Maia, C. R. Galley, A. K. Leibovich, and R. A. Porto, Radiation reaction for spinning bodies in effective field theory I: Spin-orbit effects, Phys. Rev. D 96, 084064 (2017).

[97] N. T. Maia, C. R. Galley, A. K. Leibovich, and R. A. Porto, Radiation reaction for spinning bodies in effective field theory II: Spin-spin effects, Phys. Rev. D 96, 084065 (2017).

[98] W. D. Goldberger and A. Ross, Gravitational radiative corrections from effective field theory, Phys. Rev. D 81, 124015 (2010).

[99] B. A. Pardo and N. T. Maia, Next-to-leading order spinorbit effects in the equations of motion, energy loss and phase evolution of binaries of compact bodies in the effective field theory approach, Phys. Rev. D 102, 124020 (2020).

[100] E. Racine, A. Buonanno, and L. E. Kidder, Recoil velocity at $2 \mathrm{PN}$ order for spinning black hole binaries, Phys. Rev. D 80, 044010 (2009).

[101] G. Cho and H. M. Lee, Analytic Keplerian-type parametrization for general spinning compact binaries with leading order spin-orbit interactions, Phys. Rev. D 100, 044046 (2019).

[102] K. Chatziioannou, A. Klein, N. Yunes, and N. Cornish, Constructing gravitational waves from generic spinprecessing compact binary inspirals, Phys. Rev. D 95, 104004 (2017).

[103] P. Csizmadia, G. Debreczeni, I. Racz, and M. Vasuth, Gravitational waves from spinning eccentric binaries, Classical Quantum Gravity 29, 245002 (2012).
[104] See the Supplemental Material at http://link.aps.org/ supplemental/10.1103/PhysRevD.104.024037 which contains the main results of the paper for the reader's convenience.

[105] A. Ross, Multipole expansion at the level of the action, Phys. Rev. D 85, 125033 (2012).

[106] S. Foffa and R. Sturani, Effective field theory methods to model compact binaries, Classical Quantum Gravity 31, 043001 (2014).

[107] W. Goldberger and I. Rothstein, Dissipative effects in the worldline approach to black hole dynamics, Phys. Rev. D 73, 104030 (2006).

[108] R. A. Porto, Absorption effects due to spin in the worldline approach to black hole dynamics, Phys. Rev. D 77, 064026 (2008).

[109] W. D. Goldberger, J. Li, and I.Z. Rothstein, Nonconservative effects on spinning black holes from world-line effective field theory, arXiv:2012.14869.

[110] L. Blanchet and T. Damour, Tail transported temporal correlations in the dynamics of a gravitating system, Phys. Rev. D 37, 1410 (1988).

[111] L. E. Kidder, Coalescing binary systems of compact objects to (post $)^{5 / 2}$-Newtonian order. V. Spin effects, Phys. Rev. D 52, 821 (1995).

[112] L. Blanchet, A. Buonanno, and G. Faye, Tail-induced spinorbit effect in the gravitational radiation of compact binaries, Phys. Rev. D 84, 064041 (2011).

[113] C. R. Galley and I.Z. Rothstein, Deriving analytic solutions for compact binary inspirals without recourse to adiabatic approximations, Phys. Rev. D 95, 104054 (2017).

[114] Z. Yang and A. K. Leibovich, Analytic solutions to compact binary inspirals with leading order spin-orbit contribution using the dynamical renormalization group, Phys. Rev. D 100, 084021 (2019).

[115] K. S. Thorne, Multipole expansions of gravitational radiation, Rev. Mod. Phys. 52, 299 (1980).

[116] M. J. Fitchett, The influence of gravitational wave momentum losses on the centre of mass motion of a Newtonian binay system, Mon. Not. R. Astron. Soc. 203, 1049 (1983).

[117] A. K. Leibovich, N. T. Maia, I. Z. Rothstein, and Z. Yang, Second post-Newtonian order radiative dynamics of inspiralling compact binaries in the Effective Field Theory approach, Phys. Rev. D 101, 084058 (2020).

[118] J. M. Martín-García et al., xact: Efficient tensor computer algebra for the wolfram language, http://www.xact.es/ (2019). 